\title{
ASYMPTOTIC TAIL PROBABILITIES OF RISK PROCESSES IN INSURANCE AND FINANCE
}

by

Xuemiao Hao

\author{
An Abstract \\ Of a thesis submitted in partial fulfillment of the \\ requirements for the Doctor of Philosophy \\ degree in Statistics \\ in the Graduate College of \\ The University of Iowa
}

July 2009

Thesis Supervisor: Associate Professor Qihe Tang 


\begin{abstract}
In this thesis we are interested in the impact of economic and financial factors, such as interest rate, tax payment, reinsurance, and investment return, on insurance business. The underlying risk models of insurance business that we consider range from the classical compound Poisson risk model to the newly emerging and more general Lévy risk model. In these risk models, we assume that the claim-size distribution belongs to some distribution classes according to its asymptotic tail behavior. We consider both light-tailed and heavy-tailed cases.

Our study is through asymptotic tail probabilities. Firstly, we study the asymptotic tail probability of discounted aggregate claims in the renewal risk model by introducing a constant force of interest. In this situation we focus on claims with subexponential tails. We derive for the tail probability of discounted aggregate claims an asymptotic formula, which holds uniformly for finite time intervals. For various special cases, we extend this uniformity to be valid for all time horizons.

Then, we investigate the asymptotic tail probability of the maximum exceedance of a sequence of random variables over a renewal threshold. We derive a unified asymptotic formula for this tail probability for both light-tailed and heavytailed cases.
\end{abstract}

By using the previous result, we study how to capture the impact of tax payments on the ruin probability in the Lévy risk model. We introduce periodic taxation under which the company pays tax at a fixed rate on its net income during 
each period. Assuming the Lévy measure, representing the claim-size distribution in the Lévy risk model, has a subexponential tail, a convolution-equivalent tail, or an exponential-like tail, we derive for the ruin probability several explicit asymptotic relations, in which the prefactor varies with the tax rate, reflecting the impact of tax payments.

Finally, we consider the renewal risk model in which the surplus is invested into a portfolio consisting of both a riskless bond and a risky stock. The price process of the stock is modeled by an exponential Lévy process. We derive an asymptotic formula for the tail probability of the stochastically discounted net loss process.

Abstract Approved:

Thesis Supervisor

Title and Department

Date 


\title{
ASYMPTOTIC TAIL PROBABILITIES OF RISK PROCESSES IN INSURANCE
}

\section{AND FINANCE}

\author{
by \\ Xuemiao Hao
}
A thesis submitted in partial fulfillment of the requirements for the Doctor of Philosophy degree in Statistics in the Graduate College of The University of Iowa

July 2009

Thesis Supervisor: Associate Professor Qihe Tang 
Graduate College

The University of Iowa

Iowa City, Iowa

CERTIFICATE OF APPROVAL

PH.D. THESIS

This is to certify that the Ph.D. thesis of

Xuemiao Hao

has been approved by the Examining Committee for the thesis requirement for the Doctor of Philosophy degree in Statistics at the July 2009 graduation.

Thesis Committee:

Qihe Tang, Thesis Supervisor

Palle Jorgensen

Jérôme Pansera

Elias S. W. Shiu

Nariankadu D. Shyamalkumar 


\section{ACKNOWLEDGEMENTS}

Here I want to thank all those who gave me the possibility to complete this thesis.

First and foremost, I would like to thank Professor Qihe Tang, my academic advisor. It is he who led me to research on actuarial science and guided me to find my own research interests. I really appreciate Prof. Tang's advices and encouragements in the past three years. Without his help, this work would not have been possible.

I want to express my gratitude to Professor Elias Shiu for his helpful suggestions on academic research and insightful comments in classes and seminars; to Professors Kung-Sik Chan and Jonathan Cryer for inviting me to write solutions manual for their book, from which I learned a lot; to Professor Richard Dykstra for teaching me probability courses that helped me build a solid background in probability theory. I thank the other thesis committee members, Professors Palle Jorgensen, Jérôme Pansera, and Nariankadu Shyamalkumar for their kind encouragements and supports.

My sincere thanks also go to the administrative staff of my department Margie Ebert, Dena Miller, and Tammy Siegel. Thank you for providing great support throughout my Ph.D. studies in this department. Your smiles make the main office so warm.

I am also very grateful to many of my fellow colleagues and students who

shared their thoughts and knowledge with me during my Ph.D. studies. Some of 
them are Min Jiang, Bangwon Ko, Dong Liang, Hai Liu, Jin Liu, Yan Liu, Weijie Mao, Hee Seok Nam, Andrew Ng, Mike Nielsen, Jing Pan, Fei Su, Xiongwen Tang, Li Wei, Jun Yang, Zhongyi Yuan, and Tianyang Zhang.

Finally, I want to thank my family members, particularly my wife Shu, for their everlasting support and love. To you I dedicate this thesis. 


\begin{abstract}
In this thesis we are interested in the impact of economic and financial factors, such as interest rate, tax payment, reinsurance, and investment return, on insurance business. The underlying risk models of insurance business that we consider range from the classical compound Poisson risk model to the newly emerging and more general Lévy risk model. In these risk models, we assume that the claim-size distribution belongs to some distribution classes according to its asymptotic tail behavior. We consider both light-tailed and heavy-tailed cases.

Our study is through asymptotic tail probabilities. Firstly, we study the asymptotic tail probability of discounted aggregate claims in the renewal risk model by introducing a constant force of interest. In this situation we focus on claims with subexponential tails. We derive for the tail probability of discounted aggregate claims an asymptotic formula, which holds uniformly for finite time intervals. For various special cases, we extend this uniformity to be valid for all time horizons.

Then, we investigate the asymptotic tail probability of the maximum exceedance of a sequence of random variables over a renewal threshold. We derive a unified asymptotic formula for this tail probability for both light-tailed and heavytailed cases.
\end{abstract}

By using the previous result, we study how to capture the impact of tax payments on the ruin probability in the Lévy risk model. We introduce periodic taxation under which the company pays tax at a fixed rate on its net income during 
each period. Assuming the Lévy measure, representing the claim-size distribution in the Lévy risk model, has a subexponential tail, a convolution-equivalent tail, or an exponential-like tail, we derive for the ruin probability several explicit asymptotic relations, in which the prefactor varies with the tax rate, reflecting the impact of tax payments.

Finally, we consider the renewal risk model in which the surplus is invested into a portfolio consisting of both a riskless bond and a risky stock. The price process of the stock is modeled by an exponential Lévy process. We derive an asymptotic formula for the tail probability of the stochastically discounted net loss process. 


\section{TABLE OF CONTENTS}

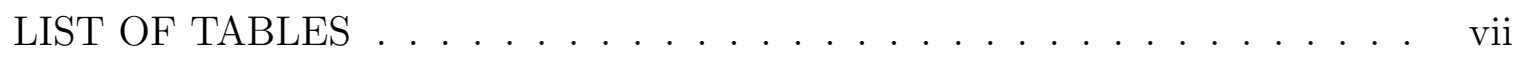

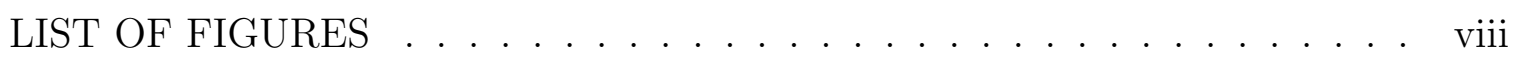
CHAPTER

1 INTRODUCTION . . . . . . . . . . . . . . . . . 1

1.1 Notation and Conventions . . . . . . . . . . . . . . . . . 1

1.2 Objectives and Outline of the Thesis . . . . . . . . . . . 3

2 PRELIMINARIES . . . . . . . . . . . . . . . . 7

2.1 Brief Review on Stochastic Processes . . . . . . . . . . . . . . . . 7

2.2 Semimartingales and Stochastic Integrals . . . . . . . . . . . . 12

2.2.1 Semimartingales . . . . . . . . . . . . . . . . 12

2.2.2 Quadratic Variation and Covariation . . . . . . . . . . . 15

2.2 .3 Stochastic Integrals . . . . . . . . . . . . . . 16

2.3 Heavy-tailed and Light-tailed Distribution Classes . . . . . . . . 20

2.3.1 Subexponentiality and Rapid Variation . . . . . . . . . . 20

2.3.2 Light-tailed Distribution Classes . . . . . . . . . . . . . 24

2.3.3 Inverse Gaussian Distributions . . . . . . . . . . . . . 26

2.3.4 Matuszewska Indices . . . . . . . . . . . . . . . . . . . . . 28

2.3.5 Other Properties .............. 30

3 DiSCOUNTED AGGREGATE CLAIMS WITH HEAVY TAILS . . . 36

3.1 Introduction . . . . . . . . . . . . . . . . . . 36

3.2 Main Results . . . . . . . . . . . . . . . . . . . . . . . 39

3.3 Proofs ................................ 42

3.3.1 Proof of Theorem 3.3 . . . . . . . . . . . . . . . . . . . 42

3.3.2 Proof of Theorem 3.4 . . . . . . . . . . . . . . . 45

3.3.3 Proof of Theorem 3.5 . . . . . . . . . . . . . . . . . . . . 49

3.3.4 Proof of Theorem 3.6 . . . . . . . . . . . . . 53

3.4 Simulation .......................... 55

4 THE MAXIMUM EXCEEDANCE OVER A RANDOM WALK . . . 57

4.1 Introduction and Main Result . . . . . . . . . . . . . . . 57

4.2 Application to Corporate Finance . . . . . . . . . . . . . . 61 
4.3 Lemmas. . . . . . . . . . . . . . . . . . . . . . . . . . . 64

4.4 Proof of Theorem 4.1(i) . . . . . . . . . . . . . . . 66

4.4 .1 Preliminary Results . . . . . . . . . . . . . 66

4.4.2 Proof of Theorem 4.1(i) for $\alpha>0 \ldots \ldots \ldots$

4.4.3 Proof of Theorem 4.1(i) for $\alpha=0 \ldots \ldots$. . . . . 70

4.5 Proof of Theorem 4.1(ii) . . . . . . . . . . . . . . . 71

4.5.1 Preliminary Results . . . . . . . . . . . . . 71

4.5.2 Proof of Theorem 4.1(ii) . . . . . . . . . . . . . 74

4.6 Simulations . . . . . . . . . . . . . . . 75

5 THE LÉVY INSURANCE RISK MODEL UNDER TAXATION . . . 79

5.1 Introduction . . . . . . . . . . . . . . . . . . . . 79

5.2 The Case of Subexponential Tails . . . . . . . . . . . . 84

5.3 The Case of Convolution-equivalent Tails . . . . . . . . . 86

5.4 The Case of Exponential-like Tails . . . . . . . . . . . . . . . 91

6 THE RENEWAL RISK MODEL WITH RISKY INVESTMENT . . . 96

6.1 Introduction . . . . . . . . . . . . . . . . 96

6.2 The Integrated Risk Process _ . . . . . . . . . . . . . . . . . . . 99

6.3 The Discounted Net Loss Process . . . . . . . . . . . . . . . . . . 101

6.4 Claims with Extended-regularly-varying Tails . . . . . . . . 105

REFERENCES . . . . . . . . . . . . . . . . . . . 110 


\section{LIST OF TABLES}

Table

2.1 Some examples of subexponential distributions . . . . . . . . . . . . . . . 21 


\section{LIST OF FIGURES}

Figure

2.1 Classes of heavy-tailed distributions . . . . . . . . . . . . . . . 31

3.1 Uniform convergence on $(0, T]$ with Pareto $F \ldots \ldots$

4.1 $y$-axis represents for each fixed $x$ the LHS substracts the RHS in (4.3) when $Y$ is $\operatorname{Exp}(1)$ distributed. . . . . . . . . . . . . . . 77

$4.2 y$-axis represents for each fixed $x$ the LHS substracts the RHS in (4.3) when $Y$ is heavy-tailed Weibull distributed. . . . . . . . . . . . . . 78

5.1 Loss-carry-forward taxation . . . . . . . . . . . . . . . . . 81

5.2 Periodic taxation . . . . . . . . . . . . . . . . . . . 82 


\section{CHAPTER 1 INTRODUCTION}

\subsection{Notation and Conventions}

Throughout this thesis we use the following conventions:

- Without otherwise stated, the limit procedure is according to $x \rightarrow \infty$.

- The summation over an empty set of indices produces a value 0 .

- The multiplication over an empty set of indices produces a value 1 .

We also use these mathematical signs:

$$
\begin{array}{ll}
a^{+} & a \vee 0=\max \{a, 0\} \\
a^{-} & -(a \wedge 0)=-\min \{a, 0\} \\
a(x) \lesssim b(x) \quad \lim \sup _{x \rightarrow \infty} a(x) / b(x) \leq 1 \text { for positive functions } a(\cdot) \text { and } b(\cdot) \\
a(x) \gtrsim b(x) \quad \liminf \operatorname{los}_{x \rightarrow \infty} a(x) / b(x) \geq 1 \text { for positive functions } a(\cdot) \text { and } b(\cdot) \\
a(x) \sim b(x) \quad \text { both relations } a(x) \lesssim b(x) \text { and } a(x) \gtrsim b(x) \text { hold } \\
a(x) \asymp b(x) \quad \limsup _{x \rightarrow \infty} a(x) / b(x)<\infty \text { and } \lim _{x \rightarrow \infty} b(x) / a(x)<\infty \\
o(1), O(1) \quad \lim _{x \rightarrow \infty} o(1)=0 \text { and } \limsup _{x \rightarrow \infty}|O(1)|<\infty
\end{array}
$$

Probability notation used is summarized below:

$1_{E} \quad$ the indicator function of an event $E$

a.s. almost surely

$\begin{array}{ll}\stackrel{d}{=} & X \stackrel{d}{=} Y \Longleftrightarrow \operatorname{Pr}(X>x)=\operatorname{Pr}(Y>x) \text { for every } x \\ \stackrel{d}{\leq} & X \stackrel{d}{\leq} Y \Longleftrightarrow \operatorname{Pr}(X>x) \leq \operatorname{Pr}(Y>x) \text { for every } x\end{array}$ 


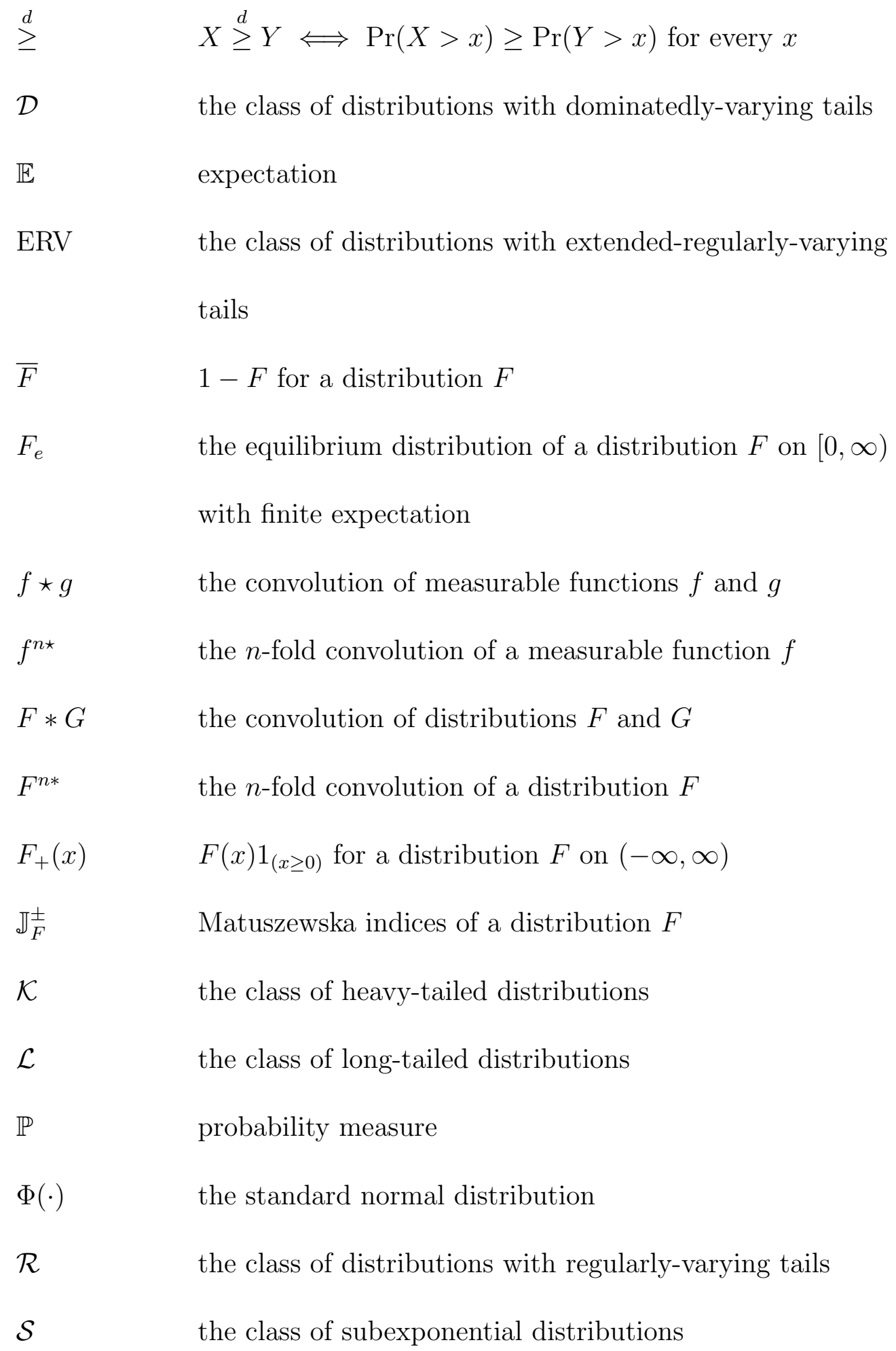

Notation for surplus process is summarized below: 


$\begin{array}{ll}B_{t} & \text { a Brownian motion at time } t \\ \text { càdlàg } & \text { right continuous with left limits } \\ D_{r}(t) & \text { the discounted aggregate claims by time } t \text { in the presence } \\ & \text { of the force of interest } r \\ L_{t} & \text { a Lévy process at time } t \\ \lambda_{t} & \text { the renewal function } \mathbb{E} N_{t} \\ \Lambda & \left\{t: \lambda_{t}>0\right\} \cup\{\infty\} \\ N_{t} & \text { a renewal counting process at time } t \\ \psi_{t} & \text { the Laplace exponent of a Lévy process } \\ \theta_{k} & \text { ruin probability of an insurance risk process } \\ U_{t} & \text { the characteristic exponent of a Lévy process } \\ & \text { the } k \text { th claim arrival time }\end{array}$

\subsection{Objectives and Outline of the Thesis}

In this thesis, we study the asymptotic tail probabilities of quantities of interest in various risk models, from the compound Poisson risk model to the newly emerging and more general Lévy risk model, to investigate the impact of economic and financial factors, such as interest rate, tax payment, reinsurance, and investment return, on insurance business. We consider both light-tailed and heavy-tailed claim sizes in our models, seeing that the tail behavior of the claim-size distribution may vary in 
different types of insurance business.

In Chapter 2 we prepare some probability tools that are needed for the following chapters. In Section 2.1 we give the definitions and properties for some stochastic processes that are widely used in insurance mathematics. In Section 2.2 we present some basic theory for stochastic integral with respect to a semimartingale. This part is crucial for us to investigate the impact of risky investment in Chapter 6. Section 2.3 reviews some popular classes of distributions, including both light-tailed and heavytailed classes. They are going to be used as assumptions on the claim-size distribution in our risk models and play a very important role in deriving our main results.

The main part of this thesis consists of Chapters 3-6. In Chapter 3 we introduce a constant force of interest in the renewal risk model and study the tail probability of discounted aggregate claims. Since it is usually not possible to get closed-form expressions except for few ideal cases, we instead aim at asymptotic formulas. The question is of much practical interest in insurance risk management. The study can provide an easy and precise approximation when measuring the risk of large losses via Value-at-Risk or Conditional Tail Expectation. Also, such an approximation usually plays a crucial role in pricing some insurance products. We derive for the tail probability of discounted aggregate claims an asymptotic formula, which holds uniformly for all time horizons. A key assumption in our model is that the claim-size distribution is subexponential.

In Chapter 4 we study an interesting problem in the field of probability that will be used as an important tool to give the proof for some main results in Chapter 
5. Motivated by the observations that many problems in applied fields, including corporate finance, insurance risk, and production systems, can be reduced to the study of the maximum exceedance of a sequence of random variables over a renewal threshold, we derive a unified asymptotic formula for the tail probability of such a maximum exceedance for both light-tailed and heavy-tailed cases.

In Chapter 5, we use a general Lévy process to model the underlying surplus process of an insurance company in a world without economic factors. This so-called Lévy risk model has recently attracted a lot of attention in the insurance literature. We are particularly interested in how to capture the impact of tax payments on the ruin probability. In a series of papers recently by Albrecher and his coauthors, it is assumed that taxes are paid at a certain fixed rate immediately when the surplus of the company is at a running maximum. In reality, however, taxes are usually paid periodically (e.g. monthly, semi-annually, or annually). Therefore, we introduce periodic taxation under which the company pays tax at a fixed rate on its net income during each period. As main results, we derive for the ruin probability several explicit asymptotic relations, in which the prefactor varies with the tax rate, reflecting the impact of tax payments.

In Chapter 6, we study the tail behavior of the stochastically discounted net loss process in the renewal risk model with risky investment. Consider an insurance company who invests its surplus into a portfolio consisting of both a riskless bond and a risky stock. Suppose the price process of the bond grows with a constant force of interest, while the price process of the stock is modeled by an exponential Lévy 
process. The study of such a risk model has become a hot topic in the past decade. Assuming a constant mix investment strategy, i.e., the proportions of surplus invested into the riskless and risky assets remain constant, we derive an asymptotic formula for the tail probability of the stochastically discounted net loss process. 


\section{CHAPTER 2 \\ PRELIMINARIES}

\subsection{Brief Review on Stochastic Processes}

Stochastic processes as a probabilistic tool have been extensively used for modeling insurance risk processes for a long time. For instance, Lundberg (1903) pointed out that Poisson processes lie at the heart of non-life insurance models.

Definition 2.1. A counting process $N=\left(N_{t}\right)_{t \geq 0}$, defined on a probability space $(\Omega, \mathcal{F}, \mathbb{P})$, is said to be a Poisson process with intensity $\lambda>0$ if

(i) the paths of $N$ are $\mathbb{P}$-almost surely right continuous with left limits (càdlàg);

(ii) $\mathbb{P}\left(N_{0}=0\right)=1$;

(iii) the process has independent increments;

(iv) the number of events in any interval of length $t$ is Poisson distributed with mean $\lambda t$, i.e., for every $s \geq 0$ and $t>0$

$$
\mathbb{P}\left(N_{t+s}-N_{s}=n\right)=\mathbb{P}\left(N_{t}=n\right)=\mathrm{e}^{-\lambda t} \frac{(\lambda t)^{n}}{n !}, \quad n=0,1, \ldots
$$

A useful property of the Poisson process with intensity $\lambda$ is that the interarrival times are independent and identically distributed (i.i.d.) exponential random variables with mean $1 / \lambda$. If we allow the inter-arrival times to be i.i.d. copies of an arbitrary nonnegative and not-degenerate-at-zero random variable (a random variable $\theta$ is said to be not degenerate at zero if $\mathbb{P}(\theta=0)<1)$, then Poisson processes are generalized to renewal counting processes. 
Definition 2.2. A counting process $N=\left(N_{t}\right)_{t \geq 0}$, defined on a probability space $(\Omega, \mathcal{F}, \mathbb{P})$, is said to be a renewal counting process with parameter $\lambda>0$ if

(i) the paths of $N$ are $\mathbb{P}$-almost surely càdlàg;

(ii) $\mathbb{P}\left(N_{0}=0\right)=1$;

(iii) the inter-arrival times are i.i.d., nonnegative, and not-degenerate-at-zero random variables with mean $1 / \lambda$.

Like Poisson processes in actuarial risk theory, Bachelier (1900) recognized that Brownian motions are a key building block for financial models.

Definition 2.3. A stochastic process $B=\left(B_{t}\right)_{t \geq 0}$, defined on a probability space $(\Omega, \mathcal{F}, \mathbb{P})$, is said to be a Brownian motion if

(i) the paths of $B$ are $\mathbb{P}$-almost surely continuous;

(ii) $\mathbb{P}\left(B_{0}=0\right)=1$;

(iii) B has stationary and independent increments;

(iv) for every $t>0, B_{t}$ is normally distributed with mean 0 and variance $\sigma^{2} t$.

When $\sigma=1$ in the above definition, the process $B$ is called a standard Brownian motion. Both Poisson processes and Brownian motions are initiated from the origin and have stationary and independent increments. Actually, these properties define a more general class of stochastic processes, which are called Lévy processes. 
Definition 2.4. A stochastic process $L=\left(L_{t}\right)_{t \geq 0}$, defined on a probability space $(\Omega, \mathcal{F}, \mathbb{P})$, is said to be a Lévy process if

(i) the paths of $L$ are $\mathbb{P}$-almost surely càdlàg;

(ii) $\mathbb{P}\left(L_{0}=0\right)=1$;

(iii) L has stationary and independent increments.

Lévy processes have an intimate relationship with infinitely divisible distributions, as described below:

Definition 2.5. A random variable $X$ is said to have an infinitely divisible distribution if for each $n=1,2, \ldots$, there exists a sequence of i.i.d. random variables $X_{1,1}, X_{1,2}, \ldots, X_{1, n}$ such that

$$
X \stackrel{d}{=} X_{1,1}+X_{1,2}+\cdots+X_{1, n}
$$

For a Lévy process $L=\left(L_{t}\right)_{t \geq 0}, L_{t}$ is a random variable with infinitely divisible distribution. This follows from the fact that for every $n=1,2, \ldots$,

$$
L_{t}=L_{t / n}+\left(L_{2 t / n}-L_{t / n}\right)+\cdots+\left(L_{t}-L_{(n-1) t / n}\right)
$$

and the fact that $L$ has stationary and independent increments. For every $t \geq 0$, define

$$
\Psi_{t}(s)=-\log \mathbb{E} \mathrm{e}^{\mathrm{i} s L_{t}}
$$

From (2.1) it is easy to obtain that for every rational $t>0$,

$$
\Psi_{t}(s)=t \Psi_{1}(s)
$$


If $t$ is an irrational number, then we can choose a decreasing sequence of rationals $\left\{t_{n}, n=1,2, \ldots\right\}$ such that $t_{n} \rightarrow t$ as $n \rightarrow \infty$. Since $L$ is almost surely right continuous, $\mathbb{E} \mathrm{e}^{\mathrm{i} s L_{t}}=\exp \left\{-\Psi_{t}(s)\right\}$ is also right continuous in $t$. Hence, (2.2) still holds.

In conclusion, for every Lévy process $L$ its characteristic function can be written in the form

$$
\mathbb{E} \mathrm{e}^{\mathrm{i} s L_{t}}=\mathrm{e}^{-t \Psi(s)},
$$

where $\Psi(s):=\Psi_{1}(s)$ is the characteristic exponent of $L_{1}$. The famous Lévy-Khintchine formula gives the following representation for $\Psi(s)$ :

$$
\Psi(s)=\mathrm{i} a s+\frac{1}{2} \sigma^{2} s^{2}+\int_{-\infty}^{\infty}\left(1-\mathrm{e}^{\mathrm{i} s x}+\mathrm{i} s x 1_{\{|x| \leq 1\}}\right) \rho(\mathrm{d} x)
$$

with $a \in(-\infty, \infty), \sigma \geq 0$, and Lévy measure $\rho$ on $(-\infty, \infty)$ satisfying $\rho(\{0\})=0$ and $\int_{-\infty}^{\infty}\left(x^{2} \wedge 1\right) \rho(\mathrm{d} x)<\infty$. The triplet $\left(a, \sigma^{2}, \rho\right)$ (called Lévy triplet) uniquely determines the distribution of the Lévy process $L$. In the following chapters, we also need the Laplace exponent of a Lévy process $L$ given by

$$
\varphi(s)=-\Psi(\mathrm{i} s)=\log \mathbb{E} \mathrm{e}^{-s L_{1}} .
$$

For more details of Lévy processes, refer to Kyprianou (2006).

Lévy processes form a very rich class of stochastic processes. Besides Poisson processes and Brownian motions, we further give the following typical examples:

\section{(i) Compound Poisson Processes}

Consider a compound Poisson process $V=\left(V_{t}\right)_{t \geq 0}$ with

$$
V_{t}=\sum_{k=1}^{N_{t}} \xi_{k}, \quad t \geq 0
$$


where $N=\left(N_{t}\right)_{t \geq 0}$ is a Poisson process with intensity $\lambda>0$ and $\xi_{1}, \xi_{2}, \ldots$ are i.i.d. random variables independent of $N$ and with common distribution $F$ on $(-\infty, \infty)$. Here, in (2.3) we use the convention that the summation over an empty set of indices produces a value 0 . For $s \in(-\infty, \infty)$,

$$
\begin{aligned}
\mathbb{E} \mathrm{e}^{\mathrm{i} s V_{t}} & =\sum_{n=0}^{\infty} \mathbb{E}\left(\mathrm{e}^{\mathrm{i} s \sum_{k=1}^{n} \xi_{k}}\right) \mathrm{e}^{-\lambda} \frac{\lambda^{n}}{n !} \\
& =\sum_{n=0}^{\infty}\left(\int_{-\infty}^{\infty} \mathrm{e}^{\mathrm{i} s x} F(\mathrm{~d} x)\right)^{n} \mathrm{e}^{-\lambda} \frac{\lambda^{n}}{n !} \\
& =\mathrm{e}^{-\lambda \int_{-\infty}^{\infty}\left(1-\mathrm{e}^{\mathrm{i} s x}\right) F(\mathrm{~d} x)} .
\end{aligned}
$$

From (2.4) we see that the Lévy triplet of the compound Poisson process $V$ is given by $a=-\lambda \int_{0<|x|<1} x F(\mathrm{~d} x), \sigma=0$, and $\rho(\mathrm{d} x)=\lambda F(\mathrm{~d} x)$.

\section{(ii) Gamma Processes}

A gamma process $\Gamma=\left(\Gamma_{t}\right)_{t \geq 0}$ is a stochastic process starting from 0 , having stationary and independent increments, and with $\Gamma_{1}$ distributed by the gamma $(\alpha, \beta)$ distribution with density

$$
f(x)=\frac{\alpha^{\beta}}{\Gamma(\beta)} x^{\beta-1} \mathrm{e}^{-\alpha x}, \quad \alpha, \beta, x>0 .
$$

Its Lévy triplet is given by $a=\beta\left(\mathrm{e}^{-\alpha}-1\right) / \alpha, \sigma=0$, and $\rho(\mathrm{d} x)=\beta x^{-1} \mathrm{e}^{-\alpha x} \mathrm{~d} x$; see Subsection 1.2.4 of Kyprianou (2006) for details.

\section{(iii) Subordinators}

A subordinator is a Lévy process whose paths are almost surely nondecreasing. The following lemma characterizes subordinators:

Lemma 2.6 (Lemma 2.14 of Kyprianou (2006)). A Lévy process is a subordinator if and only if $\rho(-\infty, 0)=0, \int_{0}^{\infty}(1 \wedge x) \rho(\mathrm{d} x)<\infty, \sigma=0$, and $a+\int_{0}^{1-} x \rho(\mathrm{d} x) \leq 0$. 


\section{(iv) Spectrally One-sided Processes}

For a Lévy process $L$, if $\rho(-\infty, 0)=0$ and $L$ is not a subordinator, then it is called a spectrally positive Lévy process. A spectrally positive Lévy process has no downward jumps. A Lévy process $L$ is called spectrally negative if $-L$ is spectrally positive. These two classes of processes are together called spectrally one-sided.

\subsection{Semimartingales and Stochastic Integrals}

In this section, we introduce stochastic integrals and semimartingales, the most general processes of which stochastic integration gives a reasonable meaning. This section is based on Chapter 8 of Klebaner (2005) and Chapter II of Protter (2005).

\subsubsection{Semimartingales}

We assume as given a complete probability space $(\Omega, \mathcal{F}, \mathbb{P})$. In addition, we are given a filtration $\mathbb{F}=\left(\mathcal{F}_{t}\right)_{0 \leq t \leq \infty}$. By a filtration we mean a family of $\sigma$-fields $\left(\mathcal{F}_{t}\right)_{0 \leq t \leq \infty}$ that is nondecreasing, i.e., $\mathcal{F}_{s} \subset \mathcal{F}_{t}$ for all $0 \leq s \leq t \leq \infty$. A nonnegative random variable $\tau$, which is allowed to take the value $\infty$, is called a stopping time (with respect to filtration $\mathbb{F}$ ) if for each $t$,

$$
\{\tau \leq t\} \in \mathcal{F}_{t}
$$

In other words, by observing the information contained in $\mathcal{F}_{t}$ we can decide whether the event $\{\tau \leq t\}$ has or has not occurred.

We then introduce finite variation. If $g$ is a function of real variable, its 
variation over the interval $[a, b]$ is defined as

$$
V_{g}([a, b])=\sup \sum_{i=1}^{n}\left|g\left(t_{i}^{n}\right)-g\left(t_{i-1}^{n}\right)\right|,
$$

where the supremum is taken over the partitions of $[a, b]$ :

$$
a=t_{0}^{n}<t_{1}^{n}<\cdots<t_{n}^{n}=b .
$$

Clearly, (by the triangle inequality) the sum in (2.5) increases as new points are added to the partitions. Therefore, the variation of $g$ is

$$
V_{g}([a, b])=\lim _{\delta_{n} \rightarrow 0} \sum_{i=1}^{n}\left|g\left(t_{i}^{n}\right)-g\left(t_{i-1}^{n}\right)\right|
$$

where $\delta_{n}=\max _{1 \leq i \leq n}\left(t_{i}-t_{i-1}\right)$. If $V_{g}([a, b])$ is finite then $g$ is said to be a function of finite variation on $[a, b]$. If $g$ is a function of $t \geq 0$, then the variation function of $g$ as a function of $t$ is defined by

$$
V_{g}(t)=V_{g}([0, t])
$$

Clearly, $V_{g}(t)$ is a nondecreasing function of $t$. The function $g$ is of finite variation if $V_{g}(t)<\infty$ for every $t$. A process $X=\left(X_{t}\right)_{t \geq 0}$ is called a finite variation process if the paths of $X$ are almost surely of finite variation.

In the following we define predictable processes and martingales:

Definition 2.7. A process $X=\left(X_{t}\right)_{t \geq 0}$ is called adapted to the filtration $\mathbb{F}=$ $\left(\mathcal{F}_{t}\right)_{0 \leq t \leq \infty}$ if for every $t, X_{t}$ is $\mathcal{F}_{t}$-measurable.

Definition 2.8. A process $H=\left(H_{t}\right)_{t \geq 0}$ is predictable if it is one of the following: 
(i) a left-continuous adapted process, in particular, a continuous adapted process;

(ii) a limit (almost sure, in probability) of left-continuous adapted processes;

(iii) a regular right-continuous process such that, for any stopping time $\tau, H \tau$

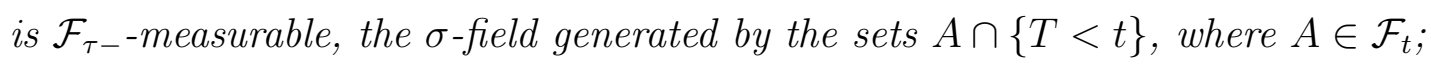

(iv) a Borel-measurable function of a predictable process.

Definition 2.9. A stochastic process $M=\left(M_{t}\right)$, where the time $t$ is continuous $0 \leq t \leq T$, or discrete $t=0,1, \ldots, T$, adapted to a filtration $\mathbb{F}$ is a martingale if for every $t, M_{t}$ is integrable (that is, $\mathbb{E}\left|M_{t}\right|<\infty$ ), and for all $0 \leq s \leq t \leq T$,

$$
\mathbb{E}\left(M_{t} \mid \mathcal{F}_{s}\right)=M_{s} \quad \text { a.s. }
$$

To define semimartingales, we still need to introduce local martingales.

Definition 2.10. A process $\left(X_{t}\right)_{0 \leq t \leq T}$ with $T \in[0, \infty]$ is called uniformly integrable if $\mathbb{E}\left(\left|X_{t}\right| 1_{\left\{\left|X_{t}\right|>n\right\}}\right)$ converges to 0 as $n \rightarrow \infty$ uniformly in $t$, that is,

$$
\lim _{n \rightarrow \infty} \sup _{0 \leq t \leq T} \mathbb{E}\left(\left|X_{t}\right| 1_{\left\{\left|X_{t}\right|>n\right\}}\right)=0
$$

where the supremum is over $[0, T]$ in the case of a finite time interval and $[0, \infty)$ if the process is considered on $0 \leq t<\infty$.

Definition 2.11. An adapted process $M=\left(M_{t}\right)_{0 \leq t \leq T}$ with $T \in[0, \infty]$ is called a local martingale if there exists a sequence of stopping times $\left\{\tau_{n}, n=1,2, \ldots\right\}$, such that $\tau_{n} \rightarrow \infty$ as $n \rightarrow \infty$ and for each $n$ the stopped process $M_{t \wedge \tau_{n}}$ is a uniformly integrable martingale in $t$. 
Now we are ready to give the definition of the core process in this section.

Definition 2.12. A càdlàg adapted process $S=\left(S_{t}\right)_{t \geq 0}$ is a semimartingale if it can be represented as a sum of two processes: a local martingale $M=\left(M_{t}\right)_{t \geq 0}$ and a finite variation process $A=\left(A_{t}\right)_{t \geq 0}$,

$$
S_{t}=S_{0}+M_{t}+A_{t}, \quad \text { with } M_{0}=A_{0}=0 .
$$

Here are some examples of semimartingales:

1. A Lévy process is a semimartingale.

2. One way to obtain semimartingale from known semimartingales is by applying a twice continuously differentiable $\left(C^{2}\right)$ transformation. If $S$ is a semimartingale and $f$ is a $C^{2}$ function, then $f(S)$ is also a semimartingale.

3. A diffusion, that is, the solution to a stochastic differential equation with respect to a Brownian motion, is a semimartingale.

\subsubsection{Quadratic Variation and Covariation}

If $X, Y$ are semimartingales on the common space, then their quadratic covariation process, also known as the square bracket process and denoted by $[X, Y](t)$, is defined, as usual, by

$$
[X, Y](t)=\lim \sum_{i=0}^{n-1}\left(X_{t_{i+1}^{n}}-X_{t_{i}^{n}}\right)\left(Y_{t_{i+1}^{n}}-Y_{t_{i}^{n}}\right),
$$

where the limit is taken over shrinking partitions $0=t_{0}^{n}<t_{1}^{n}<\ldots<t_{n}^{n}=t$ when $\delta_{n}=\max _{1 \leq i \leq n}\left(t_{i}^{n}-t_{i-1}^{n}\right) \rightarrow 0$ and is in probability. Taking $Y=X$ we obtain the quadratic variation process of $X$. 
Here are some properties of quadratic variation and covariation:

1. $[X, Y](t)$ is a regular càdlàg function of finite variation.

2. $\Delta[X, Y]=\Delta X \Delta Y$, that is, the jumps of the quadratic covariation process occur only at points where both processes have jumps.

3. If $X$ or $Y$ is of finite variation, then $[X, Y](t)=\sum_{s<t} \Delta X_{s} \Delta Y_{s}$. Notice that although the summation is taken over all $s$ not exceeding $t$, there are at most countably many terms different from zero.

4. $[X, Y]=0$ if $X$ or $Y$ is continuous and is of finite variation.

In terms of stochastic integral that to be introduced in the next subsection, we have the following lemma known as integration by parts:

Lemma 2.13. For semimartingales $X$ and $Y$, their quadratic covariation process is given by

$$
[X, Y](t)=X_{t} Y_{t}-X_{0} Y_{0}-\int_{0}^{t} X_{s-} \mathrm{d} Y_{s}-\int_{0}^{t} Y_{s-} \mathrm{d} X_{s}
$$

\subsubsection{Stochastic Integrals}

We are going to define the stochastic integral $\int_{0}^{T} H_{t} \mathrm{~d} S_{t}$ for a semimartingale $S=\left(S_{t}\right)_{t \geq 0}$. Due to (2.6) the integral with respect to $S$ is the sum of two integrals one with respect to a local martingale $M$ and the other with respect to a finite variation process $A$. The integral with respect to $A$ can be done path by path as the Stieltjes integrals, since $A$, although random, is of finite variation. So $H=\left(H_{t}\right)_{t \geq 0}$ should be 
integrable with respect to $A$. A sufficient condition for that is

$$
\int_{0}^{T}\left|H_{t}\right| V_{A}(\mathrm{~d} t)<\infty
$$

where $V_{A}(t)$ is the variation process of $A$. Then we need to define the stochastic integral of $H$ with respect to the local martingale $M, \int_{0}^{T} H_{t} \mathrm{~d} M_{t}$.

\section{Stochastic integral with respect to a martingale:}

For a simple predictable process $H$, given by

$$
H_{t}=H_{0} 1_{\{0\}}(t)+\sum_{i=0}^{n-1} h_{i} 1_{\left(T_{i}, T_{i+1}\right]}(t)
$$

where $0=T_{0} \leq T_{1} \leq \cdots \leq T_{n} \leq T$ are stopping times and $h_{i}$ is $\mathcal{F}_{T_{i}}$-measurable, $i=0,1, \ldots n-1$, the stochastic integral is defined as the sum

$$
\int_{0}^{T} H_{t} \mathrm{~d} M_{t}=\sum_{i=0}^{n-1} h_{i}\left(M_{T_{i+1}}-M_{T_{i}}\right)
$$

If $M$ is a locally square integrable martingale, then one can extend the stochastic integral from simple predictable processes to the class of predictable processes $H$ such that

$$
\sqrt{\int_{0}^{T} H_{t}^{2}[M, M](\mathrm{d} t)} \quad \text { is locally integrable. }
$$

If $M$ is a continuous local martingale, then the stochastic integral is defined for a wider class of predictable processes $H$ satisfying

$$
\int_{0}^{T} H_{t}^{2}[M, M](\mathrm{d} t) \stackrel{\text { a.s. }}{<} \infty .
$$

Stochastic Integrals with respect to a semimartingale: 
Let $S=\left(S_{t}\right)_{t \geq 0}$ be a semimartingale with representation given by (2.6). Let $H$ be a predictable process such that conditions (2.7) and (2.8) hold. Then the stochastic integral is defined as the sum of integrals,

$$
\int_{0}^{T} H_{t} \mathrm{~d} S_{t}=\int_{0}^{T} H_{t} \mathrm{~d} M_{t}+\int_{0}^{T} H_{t} \mathrm{~d} A_{t} .
$$

Although the decomposition of a semimartingale (2.6) is not unique, the stochastic integral defined above does not depend on the decomposition used. For details, see pages 216-217 of Klebaner (2005).

Since the integral with respect to a local martingale is a local martingale, and the integral with respect to a finite variation process is a finite variation process, it follows that a stochastic integral with respect to a semimartingale is still a semimartingale.

\section{Stochastic exponential:}

Definition 2.14. Let $X=\left(X_{t}\right)_{t \geq 0}$ be a semimartingale. Then the stochastic equation

$$
U_{t}=1+\int_{0}^{t} U_{s-} \mathrm{d} X_{s}
$$

has a unique solution, denoted by $\mathcal{E}(X)$, called the stochastic exponential of $X$.

For a Lévy process $L$, its ordinary exponential and stochastic exponential correspond to different stochastic processes. One may ask, which of the two processes is more suitable for building models for price dynamics. Actually, as pointed out in Subsection 8.4.3 of Cont and Tankov (2004), the two approaches are equivalent: if $Z>0$ is the stochastic exponential of a Lévy process then it is also the ordinary 
exponential of another Lévy process, and vice versa. Therefore, the two operations, although they produce different objects when applied to the same Lévy process, end up by giving us the same class of positive processes. The following lemma, due to Goll and Kallsen (2000), gives the relation between ordinary and stochastic exponentials of a Lévy process:

Lemma 2.15 (Lemma A.8 of Goll and Kallsen (2000); Proposition 8.22 of Cont and Tankov (2004)). (i) Let $X=\left(X_{t}\right)_{t \geq 0}$ be a Lévy process with Lévy triplet $\left(a, \sigma^{2}, \rho\right)$ and $Z=\mathcal{E}(X)$ its stochastic exponential. If $Z \stackrel{\text { a.s. }}{>} 0$, then there exists another Lévy process $L=\left(L_{t}\right)_{t \geq 0}$ such that $Z_{t}=\mathrm{e}^{L_{t}}$, where

$$
L_{t}=\log Z_{t}=X_{t}-\frac{\sigma^{2} t}{2}+\sum_{0 \leq s \leq t}\left(\log \left(1+\Delta X_{s}\right)-\Delta X_{s}\right)
$$

Its Lévy triplet $\left(a_{L}, \sigma_{L}^{2}, \rho_{L}\right)$ is given by

$$
\begin{aligned}
a_{L} & =a+\frac{\sigma^{2}}{2}-\int_{-\infty}^{\infty}\left(\log (1+x) 1_{\{-1 \leq \log (1+x) \leq 1\}}-x 1_{\{-1 \leq x \leq 1\}}\right) \rho(\mathrm{d} x), \\
\sigma_{L} & =\sigma \\
\rho_{L}(A) & =\rho(\{x: \log (1+x) \in A\})=\int_{-\infty}^{\infty} 1_{A} \log (1+x) \rho(\mathrm{d} x) .
\end{aligned}
$$

(ii) Let $L=\left(L_{t}\right)_{t \geq 0}$ be a Lévy process with Lévy triplet $\left(a_{L}, \sigma_{L}^{2}, \rho_{L}\right)$ and $S_{t}=\mathrm{e}^{L_{t}}$ its ordinary exponential. Then there exists a Lévy process $X=\left(X_{t}\right)_{t \geq 0}$ such that $S_{t}$ is the stochastic exponential of $X$, i.e., $S=\mathcal{E}(X)$, where

$$
X_{t}=L_{t}+\frac{\sigma^{2} t}{2}-\sum_{0 \leq s \leq t}\left(1+\Delta L_{s}-\mathrm{e}^{\Delta L_{s}}\right)
$$


The Lévy triplet $\left(a, \sigma^{2}, \rho\right)$ of $X$ is given by

$$
\begin{aligned}
a & =a_{L}-\frac{\sigma_{L}^{2}}{2}-\int_{-\infty}^{\infty}\left(\left(\mathrm{e}^{x}-1\right) 1_{\left\{-1 \leq \mathrm{e}^{x}-1 \leq 1\right\}}-x 1_{\{-1 \leq x \leq 1\}}\right) \rho_{L}(\mathrm{~d} x), \\
\sigma & =\sigma_{L} \\
\rho(A) & =\rho_{L}\left(\left\{x: \mathrm{e}^{x}-1 \in A\right\}\right)=\int_{-\infty}^{\infty} 1_{A}\left(\mathrm{e}^{x}-1\right) \rho_{L}(\mathrm{~d} x) .
\end{aligned}
$$

\subsection{Heavy-tailed and Light-tailed Distribution Classes}

\subsubsection{Subexponentiality and Rapid Variation}

Let us denote by $\mathcal{K}$ the class of (right) heavy-tailed distributions, i.e.,

$$
\mathcal{K}=\left\{F \text { distribution on }(-\infty, \infty): \int_{-\infty}^{\infty} \mathrm{e}^{\varepsilon x} F(\mathrm{~d} x)=\infty \text { for all } \varepsilon>0\right\}
$$

In insurance mathematics, claim-size distributions are often assumed to belong to some subclass of the class $\mathcal{K}$.

Next we introduce several related subclasses of the class $\mathcal{K}$. A distribution $F$ on $(-\infty, \infty)$ is said to have a long tail, denoted by $F \in \mathcal{L}$, if $\bar{F}(x)>0$ for all $x$ and

$$
\lim _{x \rightarrow \infty} \frac{\bar{F}(x-y)}{\bar{F}(x)}=1
$$

holds for all (or, equivalently, for some) $y \neq 0$.

Throughout this thesis, for two distributions $F$ and $G$ on $(-\infty, \infty)$ denote by

$$
F * G(x)=\int_{-\infty}^{\infty} F(x-y) G(\mathrm{~d} y)
$$

the convolution of $F$ and $G$. Write $F^{1 *}=F$ and $F^{n *}=F * F^{(n-1) *}$ for every $n=2,3, \ldots$ For notational convenience, write $F^{0 *}$ as a distribution degenerate at 0. A very important subclass of $\mathcal{L}$ is the subexponential class $\mathcal{S}$. By definition, a 
distribution $F$ on $[0, \infty)$ is subexponential, denoted by $F \in \mathcal{S}$, if $\bar{F}(x)>0$ for all $x \geq 0$ and the relation

$$
\lim _{x \rightarrow \infty} \frac{\overline{F^{n *}}(x)}{\bar{F}(x)}=n
$$

holds for all (or, equivalently, for some) $n=2,3, \ldots$ More generally, a distribution $F$ on $(-\infty, \infty)$ is also subexponential if $F_{+}(x)=F(x) 1_{(x \geq 0)}$ is. In this case, relation (2.11) still holds. However, for $F$ on $(-\infty, \infty)$, relation $(2.11)$ is not sufficient for $F \in \mathcal{S}$; see the example in Subsection 2.3.2.

Table 2.1, partially copied from Table 1.2.6 of Embrechts et al. (1997), gives some examples in the class $\mathcal{S}$.

\begin{tabular}{|l|l|l|}
\hline Name & Tail $\bar{F}$ or density $f$ & Parameters \\
\hline Lognormal & $f(x)=\frac{1}{\sqrt{2 \pi} \sigma x} \mathrm{e}^{-(\log x-\mu)^{2} /\left(2 \sigma^{2}\right)}$ & $-\infty<\mu<\infty, \sigma>0$ \\
\hline Pareto & $\bar{F}(x)=\left(\frac{\kappa}{\kappa+x}\right)^{\alpha}$ & $\alpha, \kappa>0$ \\
\hline Burr & $\bar{F}(x)=\left(\frac{\kappa}{\kappa+x^{\tau}}\right)^{\alpha}$ & $\alpha, \kappa, \tau>0$ \\
\hline Benktander- & $\bar{F}(x)=(1+2(\beta / \alpha) \log x)$ & $\alpha, \beta>0$ \\
type-I & $\mathrm{e}^{-\beta(\log x)^{2}-(\alpha+1) \log x}$ & \\
\hline Benktander- & $\bar{F}(x)=\mathrm{e}^{\alpha / \beta} x^{-(1-\beta)} \mathrm{e}^{-\alpha x^{\beta} / \beta}$ & $\alpha>0,0<\beta<1$ \\
\hline type-II & $\bar{F}(x)=\mathrm{e}^{-c x^{\tau}}$ & $c>0,0<\tau<1$ \\
\hline Weibull & $f(x)=\frac{\alpha^{\beta}}{\Gamma(\beta)}(\log x)^{\beta-1} x^{-\alpha-1}$ & $\alpha, \beta>0$ \\
\hline Loggamma & $\overline{2}$ & \\
\hline
\end{tabular}

Table 2.1: Some examples of subexponential distributions 
We also want to introduce some subclasses of $\mathcal{S}$ that will play important roles in the following chapters. One useful subclass is $\mathcal{A}$, which was introduced by Konstantinides et al. (2002). By definition, a distribution $F$ on $[0, \infty)$ is said to belong to the class $\mathcal{A}$ if $F \in \mathcal{S}$ and, for some $v>1$,

$$
\limsup _{x \rightarrow \infty} \frac{\bar{F}(v x)}{\bar{F}(x)}<1 .
$$

We remark that the class $\mathcal{A}$ almost coincides with the class $\mathcal{S}$. Indeed, relation (2.12) is satisfied by almost all useful distributions with unbounded supports on the right, including all distributions in Table 2.1.

Another subclass of $\mathcal{S}$ is $\mathcal{S}^{*}$, which was introduced by Klüppelberg (1988). By definition, a distribution $F$ on $[0, \infty)$ is said to belong to the class $\mathcal{S}^{*}$ if $\bar{F}(x)>0$ for all $x \geq 0, \nu_{F}=\int_{0}^{\infty} \bar{F}(x) \mathrm{d} x<\infty$, and

$$
\lim _{x \rightarrow \infty} \int_{0}^{x} \frac{\bar{F}(x-y)}{\bar{F}(x)} \bar{F}(y) \mathrm{d} y=2 \nu_{F} .
$$

It is well known that if $F \in \mathcal{S}^{*}$, then both $F \in \mathcal{S}$ and $F_{e} \in \mathcal{S}$, where $F_{e}$ is the equilibrium distribution of $F$, i.e.,

$$
F_{e}(x)=\frac{1}{\nu_{F}} \int_{0}^{x} \bar{F}(y) \mathrm{d} y, \quad x \geq 0 .
$$

For a distribution $F$ on $(-\infty, \infty)$, its equilibrium distribution $F_{e}$ is defined as the equilibrium distribution of $F_{+}$. Again, $\mathcal{S}^{*}$ contains all distributions in Table 2.1 with finite mean.

A closely related class is the class of dominatedly varying distributions. A distribution $F$ on $(-\infty, \infty)$ is said to have a dominatedly-varying tail, denoted by 
$F \in \mathcal{D}$, if $\bar{F}(x)>0$ for all $x$ and

$$
\limsup _{x \rightarrow \infty} \frac{\bar{F}(v x)}{\bar{F}(x)}<\infty
$$

holds for all (or, equivalently, for some) $0<v<1$.

$\mathcal{L} \cap \mathcal{D}$ forms another important subclass of $\mathcal{S}$; see Proposition 1.4.4 of Embrechts et al. (1997). In particular, $\mathcal{L} \cap \mathcal{D}$ covers the class ERV of distributions with extended-regularly-varying tails. By definition, a distribution $F$ on $(-\infty, \infty)$ is said to belong to the class $\operatorname{ERV}(-\alpha,-\beta)$ for some $0 \leq \alpha \leq \beta<\infty$ if $\bar{F}(x)>0$ holds for all $x$ and the relations

$$
v^{-\beta} \leq \liminf _{x \rightarrow \infty} \frac{\bar{F}(v x)}{\bar{F}(x)} \leq \limsup _{x \rightarrow \infty} \frac{\bar{F}(v x)}{\bar{F}(x)} \leq v^{-\alpha}
$$

hold for all $v \geq 1$. The class ERV means the union of all $\operatorname{ERV}(-\alpha,-\beta)$ over the range $0 \leq \alpha \leq \beta<\infty$. Note that relations (2.13) with $\alpha=\beta$ define the famous class $\mathcal{R}_{-\alpha}$ of regularly-varying-tailed distributions with regularity index $-\alpha$. Analogously, the class $\mathcal{R}$ means the union of all $\mathcal{R}_{-\alpha}$ over the range $0 \leq \alpha<\infty$.

An extension of regular variation is rapid variation. By definition, a distribution $F$ on $(-\infty, \infty)$ is said to have a rapidly-varying tail, denoted by $\mathcal{R}_{-\infty}$, if $\bar{F}(x)>0$ for all $x$ and

$$
\lim _{x \rightarrow \infty} \frac{\bar{F}(v x)}{\bar{F}(x)}=0
$$

holds for all $v>1$. Note that lognormal, Benktander-type I \& II, and Weibull distributions all belong to the class $\mathcal{R}_{-\infty}$. 


\subsubsection{Light-tailed Distribution Classes}

When the right-hand side of (2.10) is replaced by $\mathrm{e}^{\alpha y}$ for some $\alpha \geq 0$, the class $\mathcal{L}$ is generalized to the class $\mathcal{L}(\alpha)$. By definition, a distribution $F$ on $(-\infty, \infty)$ is said to belong to the class $\mathcal{L}(\alpha)$ for some $\alpha \geq 0$ if $\bar{F}(x)>0$ for all $x$ and

$$
\lim _{x \rightarrow \infty} \frac{\bar{F}(x-y)}{\bar{F}(x)}=\mathrm{e}^{\alpha y}
$$

for all $y \in(-\infty, \infty)$. For example, the exponential distribution with density $f(x)=$ $\alpha \mathrm{e}^{-\alpha x}$ for $\alpha>0$ belongs to the class $\mathcal{L}(\alpha)$. In the literature, a distribution $F$ in $\mathcal{L}(\alpha)$ with $\alpha>0$ is thus usually said to have an exponential-like tail. Furthermore, a distribution $F$ on $[0, \infty)$ is said to belong to the class $\mathcal{S}(\alpha)$ for some $\alpha \geq 0$ if $F \in \mathcal{L}(\alpha)$ and the limit

$$
\lim _{x \rightarrow \infty} \frac{\overline{F^{2 *}}(x)}{\bar{F}(x)}=2 c
$$

exists and is finite. It is known that the constant $c$ in $(2.15)$ is equal to $\int_{0-}^{\infty} \mathrm{e}^{\alpha x} F(\mathrm{~d} x)$; see Rogozin (2000) and references therein. More generally, a distribution $F$ on $(-\infty, \infty)$ is also said to belong to the class $\mathcal{S}(\alpha)$ if $F_{+}$does. In the literature, a distribution $F$ in $\mathcal{S}(\alpha)$ with $\alpha>0$ is said to have a convolution-equivalent tail.

By using a distribution in the class $\mathcal{S}(\alpha)$, we can construct an example showing that for a distribution $F$ on $(-\infty, \infty)$ relation $(2.11)$ with $n=2$ is not sufficient for $F \in \mathcal{S}$

Example. Let $Y$ be a nonnegative random variable with a distribution $G \in \mathcal{S}(\alpha)$ for some $\alpha>0$. Define

$$
X=Y-c
$$


where $c$ is the positive constant satisfying that $\mathbb{E e}^{\alpha Y}=\mathrm{e}^{c \alpha}$. Denote by $F$ the distribution of $X$. Then relation (2.11) with $n=2$ holds for $F$. Actually, as $x \rightarrow \infty$,

$$
\overline{F^{2 *}}(x)=\overline{G^{2 *}}(x+2 c) \sim 2 \mathbb{E} \mathrm{e}^{\alpha Y} \bar{G}(x+2 c) \sim 2 \mathrm{e}^{-c \alpha} \mathbb{E} \mathrm{e}^{\alpha Y} \bar{G}(x+c)=2 \bar{F}(x) .
$$

However, by Lemma 2.24(ii), $F_{+} \in \mathcal{S}(\alpha)$ because, as $x \rightarrow \infty$,

$$
\overline{F_{+}}(x)=\bar{F}(x)=\mathbb{P}(Y>x+c) \sim \mathrm{e}^{-c \alpha} \bar{G}(x)
$$

Hence, in no way can $F_{+}$belong to $\mathcal{L}$, not to say $\mathcal{S}$.

Finally, we need density classes corresponding to the classes $\mathcal{L}(\alpha)$ and $\mathcal{S}(\alpha)$. Throughout this thesis, for two measurable functions $f$ and $g:[0, \infty) \rightarrow[0, \infty)$, denote by

$$
f \star g(x)=\int_{0}^{x} f(x-y) g(y) \mathrm{d} y
$$

the convolution of $f$ and $g$. Write $f^{1 \star}=f$ and $f^{n \star}=f \star f^{(n-1) \star}$ for every $n=2,3, \ldots$. According to Chover et al. (1973) and Klüppelberg (1989), a measurable function $f:[0, \infty) \rightarrow[0, \infty)$ is said to belong to the class $\mathcal{S} d(\alpha)$ for some $\alpha \geq 0$ if $f(x)>0$ for all large $x$,

$$
\lim _{x \rightarrow \infty} \frac{f(x-y)}{f(x)}=\mathrm{e}^{\alpha y}, \quad y \in(-\infty, \infty)
$$

and

$$
\lim _{x \rightarrow \infty} \frac{f^{2 \star}(x)}{f(x)}=2 c
$$

exists and is finite. Relation (2.16) defines the class $\mathcal{L} d(\alpha)$. It is known that the constant $c$ in $(2.17)$ is equal to $\int_{0}^{\infty} \mathrm{e}^{\alpha x} f(x) \mathrm{d} x$. For a distribution $F$ with a density $f \in \mathcal{L} d(\alpha)$ for some $\alpha>0$, it is easy to see that $f(x) / \bar{F}(x) \rightarrow \alpha$. Furthermore, for 
this case $F \in \mathcal{S}(\alpha)$ if and only if $f \in \mathcal{S} d(\alpha)$. The convergence in both (2.14) and (2.16) is automatically uniform on compact $y$-intervals. See Klüppelberg (1989) for these assertions.

\subsubsection{Inverse Gaussian Distributions}

The class $\mathcal{S}(\alpha)$ with $\alpha>0$ contains inverse Gaussian distributions, which find important applications in various fields. The inverse Gaussian distributions form a two-parameter family of continuous probability distributions with support on $(0, \infty)$. The general forms of the probability density and distribution of an inverse Gaussian distribution are given by

$$
\begin{aligned}
f(x) & =\left(\frac{\nu}{2 \pi x^{3}}\right)^{1 / 2} \exp \left\{\frac{-\nu(x-\mu)^{2}}{2 \mu^{2} x}\right\}, \\
F(x) & =\Phi\left(\sqrt{\frac{\nu}{x}}\left(\frac{x}{\mu}-1\right)\right)+\exp \left\{\frac{2 \nu}{\mu}\right\} \Phi\left(-\sqrt{\frac{\nu}{x}}\left(\frac{x}{\mu}+1\right)\right),
\end{aligned}
$$

for $x>0$, where $\Phi(\cdot)$ is the standard normal distribution, $\mu>0$ is the expectation, and $\nu>0$ is the shape parameter. The inverse Gaussian distribution with parameters $\mu$ and $\nu$ is denoted by $I G(\mu, \nu)$. According to the main theorem of Embrechts (1983), $I G(\mu, \nu)$ belongs to the class $\mathcal{S}(\alpha)$ with $\alpha=\nu /\left(2 \mu^{2}\right)$.

Here are some basic properties of inverse Gaussian distributions:

1. The moment generating function of $F=I G(\mu, \nu)$ is given by

$$
M_{F}(t)=\exp \left\{\frac{\nu}{\mu}\left(1-\sqrt{1-\frac{2 \mu^{2} t}{\nu}}\right)\right\} .
$$

2. If a random variable $X$ is distributed by $I G(\mu, \nu)$, then for each $t>0$, the random variable $t X$ is distributed by $I G(t \mu, t \nu)$. 
3. If $X_{1}, \ldots, X_{n}$ are independent random variables with $X_{i}$ distributed by $I G\left(\mu_{0} w_{i}\right.$, $\left.\nu_{0} w_{i}^{2}\right), i=1, \ldots, n$, then

$$
\sum_{i=1}^{n} X_{i} \text { is distributed by } I G\left(\mu_{0} \bar{w}, \nu_{0} \bar{w}^{2}\right),
$$

where $\bar{w}=\sum_{i=1}^{n} w_{i}$.

Inverse Gaussian distributions have an intimate relationship with Brownian motions. Actually, the inverse Gaussian distributions have their origin in Brownian motions as first passage time distributions. Let $B^{(\mu)}=\left(B_{t}^{(\mu)}\right)_{t \geq 0}$ be a stochastic process such that

$$
B_{t}^{(\mu)}=\mu t+\sigma B_{t}, \quad \text { with } B_{0}^{(\mu)}=0,
$$

where $\mu$ is a constant and $\left(B_{t}\right)_{t \geq 0}$ is the standard Brownian motion. Considering

$$
T(a)=\inf \left\{t>0 \mid B_{t}^{(\mu)}=a\right\}
$$

the first passage time for a fixed level $a>0$ by $B^{(\mu)}$, we have the following results:

(i) if $\mu>0$, then $\mathbb{P}(T(a)<\infty)=1$ and

$$
T(a) \text { is distributed by } I G\left(\frac{a}{\mu}, \frac{a^{2}}{\sigma^{2}}\right) ;
$$

(ii) if $\mu<0$, then $\mathbb{P}(T(a)<\infty)=\exp \left\{2 a \mu / \sigma^{2}\right\}$ and

$$
T(a) \mid T(a)<\infty \text { is distributed by } I G\left(-\frac{a}{\mu}, \frac{a^{2}}{\sigma^{2}}\right) ;
$$

(iii) if $\mu=0$, then $\mathbb{P}(T(a)<\infty)=1$ and $T(a)$ is distributed by a stable distribution with index 1/2. (See Feller (1966, page 170) for the definition of stable distributions.) 
The above results can be proved by identifying the Laplace transform of $T(a)$ given that $T(a)<\infty$; see Prabhu (1965) and Chhikara and Folks (1989, Chapter 3$)$ for the proofs.

The inverse Gaussian distributions have been applied to a wide range of fields. Most of these applications are based on the idea of first passage times of a Brownian motion with drift. These fields include actuarial science, demography, employment management, finance, and even linguistics; see Chhikara and Folks (1989, Chapter 10), Seshadri (1999, Part II), and references therein.

\subsubsection{Matuszewska Indices}

In this subsection we introduce Matuszewska indices of a distribution, which are connected with many useful properties of heavy-tailed distribution classes. As was done by Tang and Tsitsiashvili (2003b), for each $v>0$, we set

$$
\bar{F}_{*}(v)=\liminf _{x \rightarrow \infty} \frac{\bar{F}(v x)}{\bar{F}(x)}, \quad \bar{F}^{*}(v)=\limsup _{x \rightarrow \infty} \frac{\bar{F}(v x)}{\bar{F}(x)}
$$

and then define

$$
\mathbb{J}_{F}^{+}=\inf _{v>1}\left\{-\frac{\log \bar{F}_{*}(v)}{\log v}\right\}, \quad \mathbb{J}_{F}^{-}=\sup _{v>1}\left\{-\frac{\log \bar{F}^{*}(v)}{\log v}\right\} .
$$

In the terminology of Bingham et al. (1987), $\mathbb{J}_{F}^{+}$and $\mathbb{J}_{F}^{-}$are the upper and lower Matuszewska indices of the nonnegative and nondecreasing function $f=1 / \bar{F}$. Following Tang and Tsitsiashvili (2003b), we call the quantities $\mathbb{J}_{F}^{+}$and $\mathbb{J}_{F}^{-}$the upper and lower Matuszewska indices of the distribution $F$, respectively. For more details of the Matuszewska indices, see Bingham et al. (1987, Chapter 2.1) and Cline and 
Samorodnitsky (1994). Trivially, for a distribution $F$ on $(-\infty, \infty)$ its Matuszewska indices $\mathbb{J}_{F}^{ \pm}$satisfy $0 \leq \mathbb{J}_{F}^{-} \leq \mathbb{J}_{F}^{+} \leq \infty$. The following lemma is very useful:

Lemma 2.16 (Proposition 2.2.1 of Bingham et al. (1987)). Let $F$ be a distribution on $(-\infty, \infty)$. Then,

(i) for every $\alpha^{\prime}, 0<\alpha^{\prime}<\mathbb{J}_{F}^{-} \leq \infty$, there are positive constants $c_{1}$ and $d_{1}$ such that the inequality

$$
\frac{\bar{F}(y)}{\bar{F}(x)} \leq c_{1}\left(\frac{y}{x}\right)^{-\alpha^{\prime}}
$$

holds whenever $y \geq x \geq d_{1}$;

(ii) for every $\beta^{\prime}, 0 \leq \mathbb{J}_{F}^{+}<\beta^{\prime}<\infty$, there are positive constants $c_{2}$ and $d_{2}$ such that the inequality

$$
\frac{\bar{F}(y)}{\bar{F}(x)} \geq c_{2}\left(\frac{y}{x}\right)^{-\beta^{\prime}}
$$

holds whenever $y \geq x \geq d_{2}$.

For a distribution $F \in \operatorname{ERV}(-\alpha,-\beta)$, it is easy to see that $\mathbb{J}_{F}^{-} \geq \alpha$ and $\mathbb{J}_{F}^{+} \leq \beta$. Hence, inequalities (2.19) and (2.20) apply to $F \in \operatorname{ERV}(-\alpha,-\beta)$.

Corollary 2.17. If $F \in \operatorname{ERV}(-\alpha,-\beta)$ for some $0<\alpha \leq \beta<\infty$, then for arbitrarily chosen $0<\alpha^{\prime}<\alpha \leq \beta<\beta^{\prime}<\infty$, there are some $c>0$ and $x_{0}>0$ such that

$$
\frac{\bar{F}(y)}{\bar{F}(x)} \leq c \max \left\{\left(\frac{y}{x}\right)^{-\alpha^{\prime}},\left(\frac{y}{x}\right)^{-\beta^{\prime}}\right\} \quad \text { for all } x, y \geq x_{0}
$$

Corollary 2.17 is very similar to the well-known Potter's bound for distributions in the class $\mathcal{R}$. 
Lemma 2.18 (Theorem 1.5.6 of Bingham et al. (1987)). If $F \in \mathcal{R}_{-\alpha}$ for some $\alpha \geq 0$, then for arbitrarily chosen $c>1, \delta>0$, there exists $x_{0}>0$ such that

$$
\frac{\bar{F}(y)}{\bar{F}(x)} \leq c \max \left\{\left(\frac{y}{x}\right)^{-\alpha+\delta},\left(\frac{y}{x}\right)^{-\alpha-\delta}\right\} \quad \text { for all } x, y \geq x_{0}
$$

\subsubsection{Other Properties}

The following interrelations hold for heavy-tailed distribution classes:

(a) $\mathcal{R} \subset \mathrm{ERV} \subset \mathcal{L} \cap \mathcal{D} \subset \mathcal{S} \subset \mathcal{L} \subset \mathcal{K}$;

(b) $\mathcal{D} \not \subset \mathcal{S}$ and $\mathcal{S} \not \subset \mathcal{D}$.

The inclusions $\mathcal{R} \subset$ ERV $\subset \mathcal{L} \cap \mathcal{D}$ are immediate consequences of their definitions. For the other interrelations above, see Embrechts et al. (1997) and Embrechts and Omey (1984) for detailed discussions. Figure 2.1 below clearly shows the interrelations between these heavy-tailed distribution classes.

Subexponential distributions are good candidates for modeling large claim sizes in insurance. Suppose that $X_{1}, \ldots, X_{n}$ are i.i.d. random variables with distribution $F$. Denote the partial sum by $S_{n}=X_{1}+\cdots+X_{n}$ and the maximum by $M_{n}=\max \left(X_{1}, \ldots, X_{n}\right)$. Then for every $n=2,3, \ldots$,

$$
\begin{aligned}
& \mathbb{P}\left(S_{n}>x\right)=\overline{F^{n *}}(x) \\
& \mathbb{P}\left(M_{n}>x\right)=\overline{F^{n}}(x)=\bar{F}(x) \sum_{k=0}^{n-1} F^{k}(x) \sim n \bar{F}(x) .
\end{aligned}
$$

If $F \in \mathcal{S}$, then by relation (2.11), we have

$$
\mathbb{P}\left(S_{n}>x\right) \sim \mathbb{P}\left(M_{n}>x\right)
$$




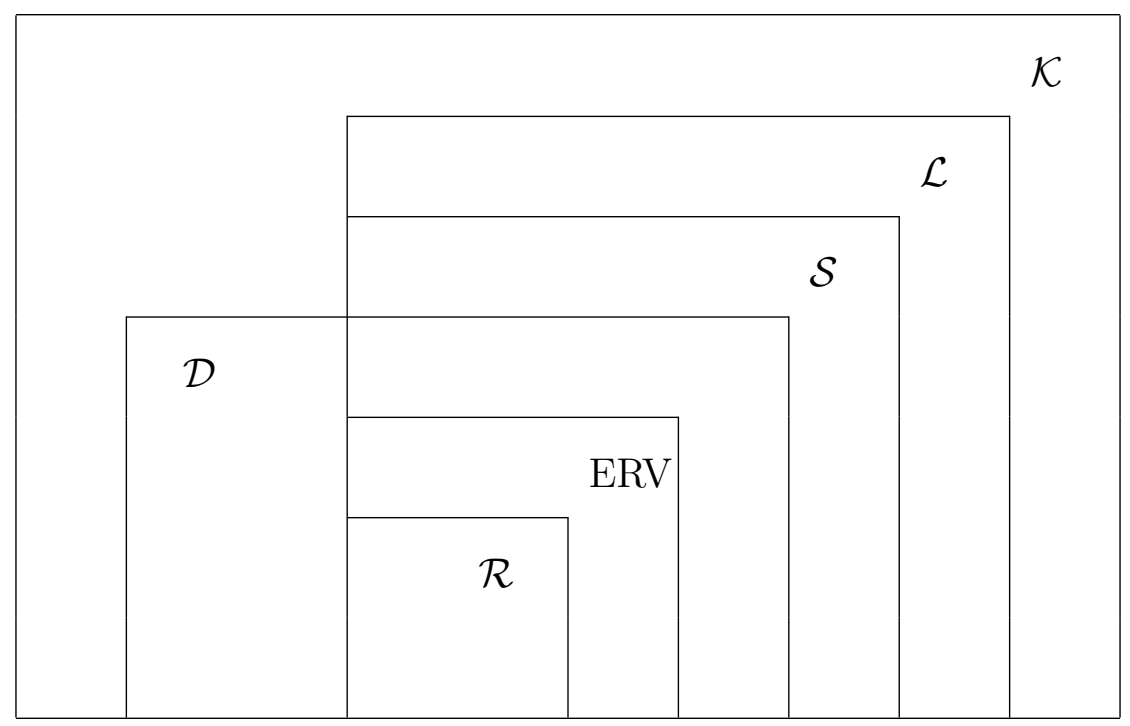

Figure 2.1: Classes of heavy-tailed distributions

which is called the principle of a single big jump in the literature. Actually, relation (2.21) is one of the intuitive notions of large claims in insurance business stating that extreme event occurs due to an unusually big jump.

This feature has been discussed in many publications. For instance, when doing a case study on the accumulated loss in the most severe storms encountered by a Swedish insurance group over the period 1982-1993, Rootzén and Tajvidi (1997) wrote that: "It can be seen that the most costly storm contributes about $25 \%$ of the total amount for the period, that it is 2.7 times bigger than the second worst storm, and that four storms together make up about half of the claims." Also, the 20-80 rule-of-thumb used by practicing actuaries when large claims are involved states that $20 \%$ of the individual claims are responsible for more than $80 \%$ of the total claim amount in a well defined portfolio. 
Next we will present several properties of the distribution classes introduced in Subsection 2.3.1 that are to be used in the following chapters. The following two lemmas are for heavy-tailed distributions:

Lemma 2.19 (Theorem 1.5.2 of Bingham et al. (1987)). If a distribution $F \in \mathcal{R}_{-\alpha}$ for some $\alpha>0$, then for every $0<a<\infty$, the convergence

$$
\lim _{x \rightarrow \infty} \frac{\bar{F}(v x)}{\bar{F}(x)}=v^{-\alpha}
$$

holds uniformly in $v$ on $[a, \infty)$, where the uniformity of the convergence means

$$
\lim _{x \rightarrow \infty} \sup _{v \geq a}\left|\frac{\bar{F}(v x)}{\bar{F}(x)}-v^{-\alpha}\right|=0
$$

Lemma 2.20 (Theorem 3.1 of Su and Tang (2003)). Consider a distribution F on $[0, \infty)$ with finite mean. If either $F \in \mathcal{L}$ or $F \in \mathcal{D}$, then the relation

$$
\lim _{x \rightarrow \infty} \frac{\bar{F}(x)}{\int_{x}^{\infty} \bar{F}(y) \mathrm{d} y}=\alpha
$$

holds with $\alpha=0$.

Then we give some properties of the classes $\mathcal{L}(\alpha)$ and $\mathcal{S}(\alpha)$ :

Lemma 2.21 (Lemma 3.1 of Tang (2007a)). For each $\alpha>0$, the following three assertions are equivalent:

$$
\text { (i) } F \in \mathcal{L}(\alpha) ; \quad \text { (ii) } \lim _{x \rightarrow \infty} \frac{\bar{F}(x)}{\int_{x}^{\infty} \bar{F}(y) \mathrm{d} y}=\alpha ; \quad \text { (iii) } F_{e} \in \mathcal{L}(\alpha) \text {. }
$$

Lemma 2.22. Assume that $F$ on $(-\infty, \infty)$ satisfies $0<\int_{0}^{\infty} \bar{F}(y) \mathrm{d} y<\infty$. For each $\alpha \geq 0, F_{e} \in \mathcal{L}(\alpha)$ if and only if relation (2.22) holds. 
Proof. For $\alpha>0$, see Lemma 2.21. For $\alpha=0$, observe that

$$
0 \leq \frac{\overline{F_{e}}(x)-\overline{F_{e}}(x+1)}{\overline{F_{e}}(x)} \leq \frac{\bar{F}(x)}{\int_{x}^{\infty} \bar{F}(y) \mathrm{d} y} \leq \frac{\overline{F_{e}}(x-1)-\overline{F_{e}}(x)}{\overline{F_{e}}(x)},
$$

from which the desired equivalence follows.

Lemma 2.23. If $F \in \mathcal{L}(\alpha)$ for some $\alpha \geq 0$, then for every $\beta>\alpha$,

(i) there exist some positive constants $c_{0}$ and $x_{0}$ such that, for all $x \geq y \geq x_{0}$,

$$
\frac{\bar{F}(y)}{\bar{F}(x)} \leq c_{0} \mathrm{e}^{\beta(x-y)}
$$

(ii) $\mathrm{e}^{-\beta x}=o(\bar{F}(x))$.

Proof. (i) Note that $F \in \mathcal{L}(\alpha)$ if and only if $\bar{F}(\ln x)$ is regularly varying of index $-\alpha$.

Thus, the desired conclusion is a straightforward consequence of Lemma 2.18.

(ii) For some $\beta^{\prime}, \alpha<\beta^{\prime}<\beta$, by item (i) there exist some positive constants $c_{0}$ and $x_{0}$ such that, for all $x \geq x_{0}$,

$$
\frac{\bar{F}\left(x_{0}\right)}{\bar{F}(x)} \leq c_{0} \mathrm{e}^{\beta^{\prime}\left(x-x_{0}\right)}
$$

Hence, the relation $\mathrm{e}^{-\beta x}=o(\bar{F}(x))$ holds.

Lemma 2.24. Let $F, G, G_{1}, G_{2}$ be distributions on $(-\infty, \infty)$.

(i) If $F \in \mathcal{L}(\alpha)$ for some $\alpha \geq 0$ and $\int_{-\infty}^{\infty} \mathrm{e}^{\beta y} G(\mathrm{~d} y)<\infty$ for some $\beta>\alpha$, then

$$
\lim _{x \rightarrow \infty} \frac{\overline{F * G}(x)}{\bar{F}(x)}=\int_{-\infty}^{\infty} \mathrm{e}^{\alpha y} G(\mathrm{~d} y) .
$$

(ii) If $F \in \mathcal{S}(\alpha)$ for some $\alpha \geq 0$ and the limit $c_{i}=\lim \overline{G_{i}}(x) / \bar{F}(x)$ exists and belongs to $[0, \infty)$ for $i=1,2$, then

$$
\lim _{x \rightarrow \infty} \frac{\overline{G_{1} * G_{2}}(x)}{\bar{F}(x)}=c_{1} \int_{-\infty}^{\infty} \mathrm{e}^{\alpha y} G_{2}(\mathrm{~d} y)+c_{2} \int_{-\infty}^{\infty} \mathrm{e}^{\alpha y} G_{1}(\mathrm{~d} y) .
$$


Proof. (i) See Lemma 2.1 of Pakes (2004). Notice that, under the current conditions, the relation $\bar{G}(x)=o(\bar{F}(x))$, as required in Lemma 2.1 of Pakes (2004), holds automatically by Lemma 2.23(ii).

(ii) See Proposition 2 of Rogozin and Sgibnev (1999).

Finally, we give two analogous upper bound properties of the distribution class $\mathcal{S}(\alpha)$ and the density class $\mathcal{S} d(\alpha)$.

Lemma 2.25. If $F \in \mathcal{S}(\alpha)$ for some $\alpha \geq 0$, then for every $n=1,2, \ldots$,

$$
\lim _{x \rightarrow \infty} \frac{\overline{F^{n *}}(x)}{\bar{F}(x)}=n\left(\int_{-\infty}^{\infty} \mathrm{e}^{\alpha y} F(\mathrm{~d} y)\right)^{n-1}
$$

Furthermore, for every $\varepsilon>0$ there exists some $K_{\varepsilon}>0$ such that for all $n=1,2, \ldots$ and $x>0$,

$$
\overline{F^{n *}}(x) \leq K_{\varepsilon}\left(\varepsilon+\int_{-\infty}^{\infty} \mathrm{e}^{\alpha y} F(\mathrm{~d} y)\right)^{n} \bar{F}(x) .
$$

Proof. For $F$ on $[0, \infty)$, relations (2.23) and (2.24) are known in Cline (1986, Corollary 1) and Embrechts and Goldie (1980). For extension to $F$ on $(-\infty, \infty)$, see Lemma 3.2 of Tang and Tsitsiashvili (2003b) for the proof of (2.23) and Lemma 5.3 of Pakes (2004) for the proof of (2.24).

Lemma 2.26 (Chover et al. (1973); Klüppelberg (1989)). If $f \in \mathcal{S} d(\alpha)$ for some $\alpha \geq 0$, then for every $n=1,2, \ldots$,

$$
\lim _{x \rightarrow \infty} \frac{f^{n \star}(x)}{f(x)}=n\left(\int_{0}^{\infty} \mathrm{e}^{\alpha y} f(y) \mathrm{d} y\right)^{n-1} .
$$

Furthermore, if $f$ is bounded, then for every $\varepsilon>0$ there exists some $K_{\varepsilon}>0$ such that 
for all $n=1,2, \ldots$ and $x>0$,

$$
f^{n \star}(x) \leq K_{\varepsilon}\left(\varepsilon+\int_{0}^{\infty} \mathrm{e}^{\alpha y} f(y) \mathrm{d} y\right)^{n} f(x) .
$$




\section{CHAPTER 3 DISCOUNTED AGGREGATE CLAIMS WITH HEAVY TAILS}

In this chapter, we introduce a constant force of interest in the renewal risk model and study the tail probability of discounted aggregate claims. Since it is usually not possible to get closed-form expressions except for few ideal cases, we instead aim at asymptotic formulas. The question is of much practical interest in insurance risk management. The study can provide an easy and precise approximation when measuring the risk of large losses via Value-at-Risk or Conditional Tail Expectation. Also, such an approximation usually plays a crucial role in pricing some insurance products. We derive for the tail probability of discounted aggregate claims an asymptotic formula, which holds uniformly for all time horizons. A key assumption in our model is that the claim-size distribution is subexponential. This chapter is based on the joint research paper Hao and Tang (2008).

\subsection{Introduction}

Consider the renewal risk model in which claim sizes $X_{k}, k=1,2, \ldots$, constitute a sequence of i.i.d. nonnegative random variables with common distribution $F$, while their arrival times $\tau_{k}, k=1,2, \ldots$, independent of $X_{k}, k=1,2, \ldots$, constitute a renewal counting process

$$
N_{t}=\#\left\{k=1,2, \ldots: \tau_{k} \leq t\right\}, \quad t \geq 0 .
$$

According to Definition 2.2, the inter-arrival times $\theta_{1}=\tau_{1}, \theta_{k}=\tau_{k}-\tau_{k-1}, k=2,3, \ldots$, constitute another sequence of i.i.d., nonnegative, and not-degenerate-at-zero random 
variables. If $\left(N_{t}\right)_{t \geq 0}$ is a Poisson process, then this model reduces to the commonly used compound Poisson model. Aggregate claims form a random sum $S_{t}=\sum_{k=1}^{N_{t}} X_{k}$, $t \geq 0$. Regarding to the distribution of $S_{t}$, we have the following lemma connecting subexponentiality and compound distributions:

Lemma 3.1 (Theorem A3.20 of Embrechts et al. (1997)). Suppose $p_{n}, n=0,1, \ldots$, form a probability measure on the set of nonnegative integers such that for some $\varepsilon>0$,

$$
\sum_{n=0}^{\infty} p_{n}(1+\varepsilon)^{n}<\infty
$$

and set

$$
G(x)=\sum_{n=0}^{\infty} p_{n} F^{n *}(x), \quad x \geq 0 .
$$

(i) If $F \in \mathcal{S}$, then $G \in \mathcal{S}$, and

$$
\lim _{x \rightarrow \infty} \frac{\bar{G}(x)}{\bar{F}(x)}=\sum_{n=1}^{\infty} n p_{n}
$$

(ii) Conversely, if (3.2) holds and there exists $n \geq 2$ such that $p_{n}>0$, then $F \in \mathcal{S}$.

For example, if $\left(N_{t}\right)_{t \geq 0}$ is a Poisson process with rate $\lambda$, then for fixed $t>0$, the tail probability of $S_{t}$ is equivalent to $\lambda \bar{F}$ as long as $F$ is subexponential.

We want to investigate the impact of interest rate on the tail behavior of the aggregate claims process. Suppose that there is a constant force of interest $r>0$. The discounted (actuarial present value of) aggregate claims are expressed as the stochastic process

$$
D_{r}(t)=\int_{0-}^{t} \mathrm{e}^{-r s} \mathrm{~d} S_{s}=\sum_{k=1}^{\infty} X_{k} \mathrm{e}^{-r \tau_{k}} 1_{\left(\tau_{k} \leq t\right)}, \quad t \geq 0
$$


From (3.3) we see that $\left(D_{r}(t)\right)_{t \geq 0}$ corresponds to a special case of the stochastic integral

$$
Z_{t}=\int_{0-}^{t} \mathrm{e}^{-R_{s}} \mathrm{~d} P_{s}, \quad t \geq 0
$$

where $\left(R_{t}\right)_{t \geq 0}$ and $\left(P_{t}\right)_{t \geq 0}$ are two independent stochastic processes fulfilling certain requirements so that $Z_{\infty}$ is well defined; see Subsection 2.2.3 for stochastic integral. When both of them are Lévy processes, Gjessing and Paulsen (1997) gave a wealth of examples showing the exact distribution or asymptotic tail probability of $Z_{\infty}$. Related discussions on the distribution of $Z_{\infty}$ can also be found in Dufresne (1990), Paulsen (1993, 1997), and Nilsen and Paulsen (1996), among others. However, we notice that all these references did not pay particular attention to the important case that $\left(P_{t}\right)_{t \geq 0}$ has heavy-tailed jumps.

In this chapter, we are interested in the asymptotic tail behavior of $D_{r}(t)$ for all $t$ for which the renewal function

$$
\lambda_{t}=\mathbb{E} N_{t}=\sum_{k=1}^{\infty} \mathbb{P}\left(\tau_{k} \leq t\right)
$$

is positive. Define $\Lambda=\left\{t: \lambda_{t}>0\right\} \cup\{\infty\}=\left\{t: \mathbb{P}\left(\tau_{1} \leq t\right)>0\right\} \cup\{\infty\}$ for later use.

For two positive bivariate functions $a(\cdot, \cdot)$ and $b(\cdot, \cdot)$, we say the asymptotic relation $a(x, t) \sim b(x, t)$ holds uniformly over all $t$ in a nonempty set $\Delta$ if

$$
\lim _{x \rightarrow \infty} \sup _{t \in \Delta}\left|\frac{a(x, t)}{b(x, t)}-1\right|=0 .
$$

Tang (2007b) investigated the tail probability of the stochastic process $D_{r}(t)$ in $(3.3)$ and gave the following result: 
Proposition 3.2 (Theorem 1.1 of Tang (2007b)). Consider the renewal risk model introduced above. If $F \in \mathrm{ERV}$, then the relation

$$
\mathbb{P}\left(D_{r}(t)>x\right) \sim \int_{0-}^{t} \bar{F}\left(x \mathrm{e}^{r s}\right) \mathrm{d} \lambda_{s}
$$

holds uniformly for all $t \in \Lambda$.

Formula (3.4) transparently captures all stochastic information of the claim sizes and their arrival times. However, we point out that the assumption $F \in$ ERV unfortunately excludes some important distributions such as lognormal and Weibull distributions. In the context of ruin theory, Tang (2005) and Wang (2008) obtained some similar asymptotic results as (3.4) for the finite-time ruin probability with a fixed time horizon $t \in \Lambda$.

Our goal in this chapter is to extend Proposition 3.2 from the class ERV to the subexponential class $\mathcal{S}$ so that lognormal and heavy-tailed Weibull distributions are included. The class ERV enjoys some favorable properties like inequalities (2.19) and (2.20), which play a crucial role in establishing Proposition 3.2, but the class $\mathcal{S}$ does not possess such properties. Therefore, to achieve the desired extension we have to employ different approaches.

The rest of this chapter consists of three sections: Section 3.2 presents our main results, Section 3.3 proves them, in turn, after preparing some necessary lemmas, and Section 3.4 gives a simulation result.

\subsection{Main Results}

The first main result of this chapter is given below: 
Theorem 3.3. Consider the discounted aggregate claims described in relation (3.3). If $F \in \mathcal{S}$, then relation (3.4) holds uniformly for all $t \in \Lambda_{T}=\Lambda \cap[0, T]$ for arbitrarily fixed finite $T \in \Lambda$.

In the next two main results below, we extend the set over which relation (3.4) holds uniformly to the maximal set $\Lambda$.

Theorem 3.4. Consider the discounted aggregate claims described in relation (3.3). If $F \in \mathcal{A}$ and $\mathbb{P}\left(\theta_{1}>\delta\right)=1$ for some $\delta>0$, then relation (3.4) holds uniformly for all $t \in \Lambda$.

Recalling the structure of $D_{r}(t)$ in (3.3), the purpose of the technical assumption on the distribution of $\theta_{1}$ in Theorem 3.4 is to let the series $D_{r}(t)$ converge more easily. Though not nice-looking, it causes no trouble for real applications since $\delta$ can be arbitrarily close to 0 . Nevertheless, we can get rid of this assumption if we slightly reduce the scope of the claim-size distribution $F$, as shown below:

Theorem 3.5. Consider the discounted aggregate claims described in relation (3.3). If $F \in \mathcal{S} \cap \mathcal{R}_{-\alpha}$ for some $0<\alpha \leq \infty$ and $\theta_{1}$ is positive, then relation (3.4) holds uniformly for all $t \in \Lambda$.

Our last main result is given below:

Theorem 3.6. Consider the discounted aggregate claims described in relation (3.3), in which $\left(N_{t}\right)_{t \geq 0}$ is a Poisson process with intensity $\lambda>0$. If $F \in \mathcal{S}$ and $F_{e} \in \mathcal{A}$, then the relation

$$
\mathbb{P}\left(D_{r}(t)>x\right) \sim \lambda \int_{0}^{t} \bar{F}\left(x \mathrm{e}^{r s}\right) \mathrm{d} s
$$


holds uniformly for all $t \in(0, \infty]$.

We remark that the assumptions $F \in \mathcal{S}$ and $F_{e} \in \mathcal{A}$ in Theorem 3.6 are satisfied by almost all useful heavy-tailed distributions such as Pareto (with finite expectation), lognormal, and heavy-tailed Weibull distributions.

Let us illustrate the usefulness of the uniformity of (3.5). Denote by

$$
\tau(x)=\inf \left\{t \geq 0: D_{r}(t)>x\right\}, \quad x>0,
$$

the time when $D_{r}(t)$ first up-crosses the level $x$. Clearly, $\tau(x)$ is a defective random variable with total mass $\mathbb{P}(\tau(x)<\infty)=\mathbb{P}\left(D_{r}(\infty)>x\right)<1$.

Let all conditions of Theorem 3.6 hold. We first consider the asymptotic behavior of the Laplace transform of $\tau(x)$. For every $u>0$, use integration by parts and the identity $\mathbb{P}(\tau(x) \leq t)=\mathbb{P}\left(D_{r}(t)>x\right)$ for all $t \geq 0$ to get

$$
\mathbb{E e}^{-u \tau(x)}=u \int_{0}^{\infty} \mathbb{P}\left(D_{r}(t)>x\right) \mathrm{e}^{-u t} \mathrm{~d} t
$$

Substituting the uniform asymptotic relation (3.5) into the above then changing the order of integrals, we have

$$
\mathbb{E} \mathrm{e}^{-u \tau(x)} \sim \lambda \int_{0}^{\infty} \mathrm{e}^{-u s \bar{F}}\left(x \mathrm{e}^{r s}\right) \mathrm{d} s
$$

This gives an explicit asymptotic expression for the Laplace transform of $\tau(x)$.

We then consider the limiting distribution of $\tau(x)$ conditional on $(\tau(x)<\infty)$.

For every fixed $t>0$, by Theorem 3.6,

$$
\mathbb{P}(\tau(x) \leq t \mid \tau(x)<\infty)=\frac{\mathbb{P}\left(D_{r}(t)>x\right)}{\mathbb{P}\left(D_{r}(\infty)>x\right)} \sim \frac{\int_{0}^{t} \bar{F}\left(x \mathrm{e}^{r s}\right) \mathrm{d} s}{\int_{0}^{\infty} \bar{F}\left(x \mathrm{e}^{r s}\right) \mathrm{d} s}
$$


If $F \in \mathcal{R}_{-\alpha}$ for some $\alpha>0$, then using Lemma 2.19 we see that the convergence

$$
\frac{\bar{F}\left(x \mathrm{e}^{r s}\right)}{\bar{F}(x)} \rightarrow \mathrm{e}^{-\alpha r s}
$$

holds uniformly for all $s \in[0, \infty)$. Therefore, dividing both integrands on the righthand side of (3.6) by $\bar{F}(x)$ then plugging (3.7), we obtain

$$
\mathbb{P}(\tau(x) \leq t \mid \tau(x)<\infty) \rightarrow 1-\mathrm{e}^{-\alpha r t}
$$

meaning that the limiting distribution under discussion is exponential.

\subsection{Proofs}

\subsubsection{Proof of Theorem 3.3}

Lemma 3.7. Let $X_{1}, \ldots, X_{n}$ be $n$ independent random variables, each $X_{k}$ distributed by $F_{k}$. If there are $n$ positive constants $l_{1}, \ldots, l_{n}$, and a distribution $F \in \mathcal{S}$ such that $\overline{F_{k}}(x) \sim l_{k} \bar{F}(x)$ holds for $k=1, \ldots, n$, then for arbitrarily fixed numbers a and $b$, $0<a \leq b<\infty$, the relation

$$
\mathbb{P}\left(\sum_{k=1}^{n} c_{k} X_{k}>x\right) \sim \sum_{k=1}^{n} \overline{F_{k}}\left(x / c_{k}\right)
$$

holds uniformly for all $\left(c_{1}, \ldots, c_{n}\right) \in[a, b] \times \cdots \times[a, b]$.

Proof. The proof can be given by going along the same lines of the proof of Proposition 5.1 of Tang and Tsitsiashvili (2003a) with some obvious modifications.

Lemma 3.8 (Lemma 2.2 of Cai and Kalashnikov (2000)). Consider the renewal counting process $\left(N_{t}\right)_{t \geq 0}$ defined in (3.1). For any $t_{1} \geq 0, t_{2} \geq 0$, there exists a random variable $\widehat{N}_{t_{2}}$ such that $\widehat{N}_{t_{2}}$ and $N_{t_{1}}$ are independent, $\widehat{N}_{t_{2}} \stackrel{d}{=} N_{t_{2}}$, and

$$
N_{t_{1}+t_{2}} \leq N_{t_{1}}+\widehat{N}_{t_{2}}+1
$$


Lemma 3.9. Consider the renewal counting process $\left(N_{t}\right)_{t \geq 0}$ defined in (3.1). There exists some $h>0$ such that $\mathbb{E} \mathrm{e}^{h N_{t}}<\infty$ holds for all $t \geq 0$.

Proof. It is shown in Stein (1946) that, for arbitrarily fixed $t_{0}>0$, there exists some $h>0$ such that $\mathbb{E}^{h N_{t_{0}}}<\infty$. For every $t \geq 0$, we can find a positive integer $k$ such that $(k-1) t_{0} \leq t<k t_{0}$. Inductively applying Lemma 3.8, we can obtain i.i.d. random variables $\widehat{N}_{t_{0}}(1), \ldots, \widehat{N}_{t_{0}}(k)$ with common distribution as that of $N_{t_{0}}$ such that

$$
N_{t} \leq N_{k t_{0}} \leq \sum_{i=1}^{k} \widehat{N}_{t_{0}}(i)+k-1
$$

where for two random variables $X$ and $Y$, the relation $X \stackrel{d}{\leq} Y$ means that $\mathbb{P}(X>$ $x) \leq \mathbb{P}(Y>x)$ for all $x$. Therefore, $\mathbb{E} \mathrm{e}^{h N_{t}}<\infty$, as claimed.

Now we are ready to give the proof for Theorem 3.3.

Proof of Theorem 3.3. Arbitrarily choose some positive integer $N$. Clearly, for $t \in \Lambda_{T}$

$$
\begin{aligned}
\mathbb{P}\left(D_{r}(t)>x\right) & =\left(\sum_{n=1}^{N}+\sum_{n=N+1}^{\infty}\right) \mathbb{P}\left(\sum_{k=1}^{n} X_{k} \mathrm{e}^{-r \tau_{k}}>x, N_{t}=n\right) \\
& =I_{1}(x, t, N)+I_{2}(x, t, N) .
\end{aligned}
$$


First consider $I_{2}(x, t, N)$. We have

$$
\begin{aligned}
I_{2}(x, t, N) & \leq \sum_{n=N+1}^{\infty} \mathbb{P}\left(\sum_{k=1}^{n} X_{k} \mathrm{e}^{-r \tau_{1}}>x, \tau_{n} \leq t<\tau_{n+1}\right) \\
& =\sum_{n=N+1}^{\infty} \int_{0-}^{t} \mathbb{P}\left(\sum_{k=1}^{n} X_{k} \mathrm{e}^{-r s}>x, \tau_{n}-\tau_{1} \leq t-s<\tau_{n+1}-\tau_{1}\right) \mathbb{P}\left(\tau_{1} \in \mathrm{d} s\right) \\
& =\sum_{n=N+1}^{\infty} \int_{0-}^{t} \mathbb{P}\left(\sum_{k=1}^{n} X_{k}>x \mathrm{e}^{r s}\right) \mathbb{P}\left(N_{t-s}=n-1\right) \mathbb{P}\left(\tau_{1} \in \mathrm{d} s\right) \\
& \leq \sum_{n=N}^{\infty} \int_{0-}^{t} \mathbb{P}\left(\sum_{k=1}^{n+1} X_{k}>x \mathrm{e}^{r s}\right) \mathbb{P}\left(N_{t-s}=n\right) \mathrm{d} \lambda_{s} .
\end{aligned}
$$

Applying Lemma 2.25 to the above, for every $\varepsilon>0$ and some $c_{\varepsilon}>0$,

$$
\begin{aligned}
I_{2}(x, t, N) & \leq c_{\varepsilon}(1+\varepsilon) \int_{0-}^{t} \bar{F}\left(x \mathrm{e}^{r s}\right) \mathbb{E}(1+\varepsilon)^{N_{t-s}} 1_{\left\{N_{t-s} \geq N\right\}} \mathrm{d} \lambda_{s} \\
& \leq c_{\varepsilon}(1+\varepsilon) \mathbb{E}(1+\varepsilon)^{N_{T}} 1_{\left\{N_{T} \geq N\right\}} \int_{0-}^{t} \bar{F}\left(x \mathrm{e}^{r s}\right) \mathrm{d} \lambda_{s} .
\end{aligned}
$$

By Lemma 3.9, we can choose some $\varepsilon$ sufficiently small such that $\mathbb{E}(1+\varepsilon)^{N_{T}}<\infty$. It follows that $\mathbb{E}(1+\varepsilon)^{N_{T}} 1_{\left\{N_{T} \geq N\right\}} \rightarrow 0$ as $N \rightarrow \infty$. Therefore, for all $x>0$,

$$
\lim _{N \rightarrow \infty} \sup _{t \in \Lambda_{T}} \frac{I_{2}(x, t, N)}{\int_{0-}^{t} \bar{F}\left(x \mathrm{e}^{r s}\right) \mathrm{d} \lambda_{s}}=0
$$

Next consider $I_{1}(x, t, N)$. Using Lemma 3.7, it holds uniformly for all $t \in \Lambda_{T}$ that

$$
\begin{aligned}
I_{1}(x, t, N) & \sim\left(\sum_{n=1}^{\infty} \sum_{k=1}^{n}-\sum_{n=N+1}^{\infty} \sum_{k=1}^{n}\right) \mathbb{P}\left(X_{k} \mathrm{e}^{-r \tau_{k}}>x, N_{t}=n\right) \\
& =I_{11}(x, t)-I_{12}(x, t, N) .
\end{aligned}
$$

Clearly, for all $t \in \Lambda_{T}$,

$$
I_{11}(x, t)=\sum_{k=1}^{\infty} \mathbb{P}\left(X_{k} \mathrm{e}^{-r \tau_{k}}>x, N_{t} \geq k\right)=\int_{0-}^{t} \bar{F}\left(x \mathrm{e}^{r s}\right) \mathrm{d} \lambda_{s} .
$$


For $I_{12}(x, t, N)$, similarly to the derivation for $I_{2}(x, t, N)$, we have

$$
\begin{aligned}
I_{12}(x, t, N) & \leq \sum_{n=N+1}^{\infty} \sum_{k=1}^{n} \mathbb{P}\left(X_{k} \mathrm{e}^{-r \tau_{1}}>x, N_{t}=n\right) \\
& \leq \sum_{n=N}^{\infty} \sum_{k=1}^{n+1} \int_{0-}^{t} \bar{F}\left(x \mathrm{e}^{r s}\right) \mathbb{P}\left(N_{t-s}=n\right) \mathrm{d} \lambda_{s} \\
& \leq \int_{0-}^{t} \bar{F}\left(x \mathrm{e}^{r s}\right) \mathrm{d} \lambda_{s} \sum_{n=N}^{\infty}(n+1) \mathbb{P}\left(N_{T} \geq n\right) .
\end{aligned}
$$

It follows that, for all $x>0$,

$$
\lim _{N \rightarrow \infty} \sup _{t \in \Lambda_{T}} \frac{I_{12}(x, t, N)}{\int_{0-}^{t} \bar{F}\left(x \mathrm{e}^{r s}\right) \mathrm{d} \lambda_{s}}=0
$$

From (3.8), (3.9), and (3.10) we conclude that the asymptotic relation (3.4) holds uniformly for all $t \in \Lambda_{T}$.

\subsubsection{Proof of Theorem 3.4}

Lemma 3.10. If a distribution $F$ on $[0, \infty)$ satisfies (2.12) for some $v>1$, then

$$
\lim _{t \rightarrow \infty} \limsup _{x \rightarrow \infty} \frac{\int_{t}^{\infty} \bar{F}\left(x \mathrm{e}^{r s}\right) \mathrm{d} \lambda_{s}}{\int_{0}^{t} \bar{F}\left(x \mathrm{e}^{r s}\right) \mathrm{d} \lambda_{s}}=0
$$

where the positive constant $r$ and the renewal function $\lambda_{s}, s \geq 0$, are the same as introduced in Section 3.1.

Proof. For every $t \in \Lambda$, apply inequality (2.19) to obtain that, for $x \geq x_{0}$,

$$
\begin{aligned}
\frac{\int_{t}^{\infty} \bar{F}\left(x \mathrm{e}^{r s}\right) \mathrm{d} \lambda_{s}}{\int_{0}^{t} \bar{F}\left(x \mathrm{e}^{r s}\right) \mathrm{d} \lambda_{s}} & =\frac{\int_{t}^{\infty} \bar{F}\left(x \mathrm{e}^{r s}\right) / \bar{F}\left(x \mathrm{e}^{r t}\right) \mathrm{d} \lambda_{s}}{\int_{0}^{t} \bar{F}\left(x \mathrm{e}^{r s}\right) / \bar{F}\left(x \mathrm{e}^{r t}\right) \mathrm{d} \lambda_{s}} \\
& \leq c^{2} \frac{\int_{t}^{\infty} \mathrm{e}^{-p r(s-t)} \mathrm{d} \lambda_{s}}{\int_{0}^{t} \mathrm{e}^{p r(t-s)} \mathrm{d} \lambda_{s}}=c^{2} \frac{\int_{t}^{\infty} \mathrm{e}^{-p r s} \mathrm{~d} \lambda_{s}}{\int_{0}^{t} \mathrm{e}^{-p r s} \mathrm{~d} \lambda_{s}}
\end{aligned}
$$

This implies (3.11). 
Lemma 3.11 (Corollary 3.1 of Chen et al. (2005)). Let $X_{k}, k=1,2, \ldots$, be a sequence of i.i.d. nonnegative random variables with common distribution $F \in \mathcal{A}$. If

$$
\sum_{k=1}^{\infty} \omega_{k}^{\delta}<\infty, \quad \text { for some } 0<\delta<\frac{\mathbb{J}_{F}^{-}}{1+\mathbb{J}_{F}^{-}}
$$

where $\mathbb{J}_{F}^{-}$is the lower Matuszewska index of $F$, then

$$
\mathbb{P}\left(\sum_{k=1}^{\infty} \omega_{k} X_{k}>x\right) \sim \sum_{k=1}^{\infty} \mathbb{P}\left(\omega_{k} X_{k}>x\right)
$$

Before presenting the following lemmas, we need to define the association of random variables. We use the definition given by Esary et al. (1967) here:

Definition 3.12. Random variables $X_{1}, \ldots, X_{n}$ are associated if

$$
\operatorname{Cov}(f(\mathbf{X}), g(\mathbf{X})) \geq 0, \quad \text { where } \mathbf{X}=\left(X_{1}, \ldots, X_{n}\right)
$$

for all nondecreasing functions $f$ and $g$ for which $\mathbb{E} f(\mathbf{X}), \mathbb{E} g(\mathbf{X})$, and $\mathbb{E} f(\mathbf{X}) g(\mathbf{X})$ exist.

Lemma 3.13 (Theorem 2.1 of Esary et al. (1967)). Independent random variables are associated.

Lemma 3.14. Under the conditions of Theorem 3.4, we have

$$
\mathbb{P}\left(D_{r}(\infty)>x\right) \lesssim \int_{0}^{\infty} \bar{F}\left(x \mathrm{e}^{r s}\right) \mathrm{d} \lambda_{s}
$$

Proof. Arbitrarily choose some positive integer $N$ such that $N \delta \in \Lambda$. Since $\mathbb{P}\left(\theta_{1}>\delta\right)=$ 1, we have

$$
\mathbb{P}\left(D_{r}(\infty)>x\right) \leq \mathbb{P}\left(\sum_{k=1}^{N} X_{k} \mathrm{e}^{-r \tau_{k}}+\left(\sum_{k=N+1}^{\infty} X_{k} \mathrm{e}^{-r(k-N) \delta}\right) \mathrm{e}^{-r \tau_{N}}>x\right)
$$


Write $\Sigma_{\delta}=\sum_{k=N+1}^{\infty} X_{k} \mathrm{e}^{-r(k-N) \delta}$, whose distribution does not depend on $N$. Applying Lemma 3.11,

$$
\mathbb{P}\left(\Sigma_{\delta}>x\right)=\mathbb{P}\left(\sum_{k=1}^{\infty} X_{k} \mathrm{e}^{-r k \delta}>x\right) \sim \bar{F}(x) \sum_{k=1}^{\infty} \frac{\bar{F}\left(x \mathrm{e}^{r k \delta}\right)}{\bar{F}(x)} .
$$

Hence, by inequality $(2.19)$, there is some constant $c_{*}>0$ such that $\mathbb{P}\left(\Sigma_{\delta}>x\right) \leq$ $c_{*} \bar{F}(x)$ for all $x \in[0, \infty)$. Next we come back to (3.13). Introduce a new random variable $\widetilde{\Sigma_{\delta}}$ independent of $\left\{X_{k}, k=1,2, \ldots\right\}$ and $\left\{\tau_{k}, k=1,2, \ldots\right\}$ with a tail satisfying

$$
\mathbb{P}\left(\widetilde{\Sigma_{\delta}}>x\right)=\min \left\{C_{*} \bar{F}(x), 1\right\}, \quad x \geq 0
$$

Therefore, $\Sigma_{\delta} \stackrel{d}{\leq} \widetilde{\Sigma_{\delta}}$, and

$$
\mathbb{P}\left(D_{r}(\infty)>x\right) \leq \mathbb{P}\left(\sum_{k=1}^{N} X_{k} \mathrm{e}^{-r \tau_{k}}+\widetilde{\Sigma_{\delta}} \mathrm{e}^{-r \tau_{N}}>x\right)
$$

To apply Lemma 3.7 , we choose some $M_{1}>0$ and derive

$$
\begin{aligned}
\mathbb{P}\left(\sum_{k=1}^{N} X_{k} \mathrm{e}^{-r \tau_{k}}+\widetilde{\Sigma_{\delta}} \mathrm{e}^{-r \tau_{N}}>x\right)= & \mathbb{P}\left(\sum_{k=1}^{N} X_{k} \mathrm{e}^{-r \tau_{k}}+\widetilde{\Sigma_{\delta}} \mathrm{e}^{-r \tau_{N}}>x, \bigcup_{i=1}^{N}\left(\theta_{i} \geq M_{1}\right)\right) \\
& +\mathbb{P}\left(\sum_{k=1}^{N} X_{k} \mathrm{e}^{-r \tau_{k}}+\widetilde{\Sigma_{\delta}} \mathrm{e}^{-r \tau_{N}}>x, \bigcap_{i=1}^{N}\left(\theta_{i}<M_{1}\right)\right) \\
= & J_{1}\left(x, N, M_{1}\right)+J_{2}\left(x, N, M_{1}\right) .
\end{aligned}
$$

Since $\theta_{1}, \ldots, \theta_{N}$ are i.i.d. random variables, by Lemma 3.13 they are associated. By the definition of association we have

$$
J_{1}\left(x, N, M_{1}\right) \leq \mathbb{P}\left(\sum_{k=1}^{N} X_{k} \mathrm{e}^{-r \tau_{k}}+\widetilde{\Sigma_{\delta}} \mathrm{e}^{-r \tau_{N}}>x\right) \mathbb{P}\left(\bigcup_{i=1}^{N}\left(\theta_{i} \geq M_{1}\right)\right)
$$

Substituting (3.16) into (3.15) and rearranging the resulting inequality, we have

$$
\mathbb{P}\left(\sum_{k=1}^{N} X_{k} \mathrm{e}^{-r \tau_{k}}+\widetilde{\Sigma_{\delta}} \mathrm{e}^{-r \tau_{N}}>x\right) \leq \frac{J_{2}\left(x, N, M_{1}\right)}{1-\mathbb{P}\left(\bigcup_{i=1}^{N}\left(\theta_{i} \geq M_{1}\right)\right)}
$$


Further substituting this into (3.14), applying Lemma 3.7 to $J_{2}\left(x, N, M_{1}\right)$, and letting $M_{1} \rightarrow \infty$, we obtain that

$$
\begin{aligned}
\mathbb{P}\left(D_{r}(\infty)>x\right) & \lesssim \sum_{k=1}^{N} \mathbb{P}\left(X_{k} \mathrm{e}^{-r \tau_{k}}>x\right)+\mathbb{P}\left(\widetilde{\Sigma_{\delta}} \mathrm{e}^{-r \tau_{N}}>x\right) \\
& \leq \sum_{k=1}^{\infty} \mathbb{P}\left(X_{k} \mathrm{e}^{-r \tau_{k}}>x\right)+\int_{N \delta}^{\infty} \mathbb{P}\left(\widetilde{\Sigma_{\delta}}>x \mathrm{e}^{r s}\right) \mathbb{P}\left(\tau_{N} \in \mathrm{d} s\right) \\
& \leq \int_{0}^{\infty} \bar{F}\left(x \mathrm{e}^{r s}\right) \mathrm{d} \lambda_{s}+c_{*} \int_{N \delta}^{\infty} \bar{F}\left(x \mathrm{e}^{r s}\right) \mathbb{P}\left(\tau_{N} \in \mathrm{d} s\right) .
\end{aligned}
$$

Apply inequality (2.19) again to obtain that, for some $M_{2} \in \Lambda \cap(0, N \delta]$ and all large $x$,

$$
\begin{aligned}
\frac{\int_{N \delta}^{\infty} \bar{F}\left(x \mathrm{e}^{r s}\right) \mathbb{P}\left(\tau_{N} \in \mathrm{d} s\right)}{\int_{0}^{\infty} \bar{F}\left(x \mathrm{e}^{r s}\right) \mathrm{d} \lambda_{s}} & \leq \frac{c \bar{F}\left(x \mathrm{e}^{r M_{2}}\right) \mathbb{E} \mathrm{e}^{-p r\left(\tau_{N}-M_{2}\right)}}{\int_{0}^{M_{2}} \bar{F}\left(x \mathrm{e}^{r s}\right) \mathrm{d} \lambda_{s}} \\
& \leq \frac{c}{\lambda_{M_{2}}} \mathbb{E} \mathrm{e}^{-p r\left(\tau_{N}-M_{2}\right)} \rightarrow 0
\end{aligned}
$$

as $N \rightarrow \infty$. From (3.17) and (3.18), the asymptotic relation (3.12) follows immediately.

Now we are ready to give the proof for Theorem 3.4.

Proof of Theorem 3.4. According to Lemma 3.10, for every $\varepsilon>0$ there exists some $T_{0}>0$ such that the inequality

$$
\int_{T_{0}}^{\infty} \bar{F}\left(x \mathrm{e}^{r s}\right) \mathrm{d} \lambda_{s} \leq \varepsilon \int_{0}^{T_{0}} \bar{F}\left(x \mathrm{e}^{r s}\right) \mathrm{d} \lambda_{s}
$$

holds for all large $x$. By Theorem 3.3 and inequality (3.19), it holds uniformly for all 
$t \in\left(T_{0}, \infty\right]$ that

$$
\begin{aligned}
\mathbb{P}\left(D_{r}(t)>x\right) & \geq \mathbb{P}\left(D_{r}\left(T_{0}\right)>x\right) \\
& \sim \int_{0}^{T_{0}} \bar{F}\left(x \mathrm{e}^{r s}\right) \mathrm{d} \lambda_{s} \\
& \geq\left(\int_{0}^{t}-\int_{T_{0}}^{\infty}\right) \bar{F}\left(x \mathrm{e}^{r s}\right) \mathrm{d} \lambda_{s} \\
& \geq(1-\varepsilon) \int_{0}^{t} \bar{F}\left(x \mathrm{e}^{r s}\right) \mathrm{d} \lambda_{s} .
\end{aligned}
$$

Likewise, by Lemma 3.14 and inequality (3.19), it holds uniformly for all $t \in\left(T_{0}, \infty\right]$ that

$$
\begin{aligned}
\mathbb{P}\left(D_{r}(t)>x\right) & \leq \mathbb{P}\left(D_{r}(\infty)>x\right) \\
& \lesssim \int_{0}^{\infty} \bar{F}\left(x \mathrm{e}^{r s}\right) \mathrm{d} \lambda_{s} \\
& \leq\left(\int_{0}^{t}+\int_{T_{0}}^{\infty}\right) \bar{F}\left(x \mathrm{e}^{r s}\right) \mathrm{d} \lambda_{s} \\
& \leq(1+\varepsilon) \int_{0}^{t} \bar{F}\left(x \mathrm{e}^{r s}\right) \mathrm{d} \lambda_{s} .
\end{aligned}
$$

Hence, for all $t \in\left(T_{0}, \infty\right]$ and all large $x$,

$$
(1-2 \varepsilon) \int_{0}^{t} \bar{F}\left(x \mathrm{e}^{r s}\right) \mathrm{d} \lambda_{s} \leq \mathbb{P}\left(D_{r}(t)>x\right) \leq(1+2 \varepsilon) \int_{0}^{t} \bar{F}\left(x \mathrm{e}^{r s}\right) \mathrm{d} \lambda_{s} .
$$

By Theorem 3.3 again, the inequalities in (3.20) also hold for all $t \in \Lambda_{T_{0}}$ (hence for all $t \in \Lambda$ ) and all large $x$. As $\varepsilon>0$ is arbitrary, we complete the proof.

\subsubsection{Proof of Theorem 3.5}

For a distribution $F \in \mathcal{R}_{-\alpha}$ for some $0<\alpha \leq \infty$, relation (2.12) obviously holds. Hence, Lemma 3.10 still works under the conditions of Theorem 3.5. We need to prepare the following lemma to replace Lemma 3.14: 
Lemma 3.15. Consider the discounted aggregate claims described in relation (3.3). If $F \in \mathcal{S} \cap \mathcal{R}_{-\alpha}$ for some $0<\alpha \leq \infty$ and $\theta_{1}$ is positive, then we have

$$
\mathbb{P}\left(D_{r}(\infty)>x\right) \sim \int_{0}^{\infty} \bar{F}\left(x \mathrm{e}^{r s}\right) \mathrm{d} \lambda_{s}
$$

Proof. When $F \in \mathcal{R}_{-\alpha}$ for some $0<\alpha<\infty$, relation (3.21) holds by Proposition 3.2. We only need to consider the case $F \in \mathcal{S} \cap \mathcal{R}_{-\infty}$.

The following proof is based on the work of Konstantinides and Tang (2009). Denote $Y_{k}=\mathrm{e}^{-r \theta_{k}}, k=1,2, \ldots$ Then $\left(X_{k}, Y_{k}\right), k=1,2, \ldots$, are i.i.d. random copies, say, of the generic random pair $(X, Y)$. It is clear that

$$
D_{r}(\infty)=\sum_{k=1}^{\infty} X_{k} \prod_{i=1}^{k} Y_{i}
$$

Furthermore, $D_{r}(\infty)$ satisfies the random functional equation

$$
R \stackrel{d}{=} Y(X+R)
$$

where $R$ is a random variable independent of $(X, Y)$.

We turn to find out the asymptotic tail probability of the solution $R$ for the random equation (3.23). From (3.22) it is obvious that

$$
\mathbb{P}(R>x) \geq \mathbb{P}\left(X_{1} Y_{1}>x\right), \quad-\infty<x<\infty
$$

For the upper bound, we consider the random difference equation

$$
R_{n+1}=Y_{n+1}\left(X_{n+1}+R_{n}\right), \quad n=0,1,2, \ldots
$$

where $R_{0}$ is independent of $\left\{\left(X_{k}, Y_{k}\right), k=1,2, \ldots\right\}$. Since $Y \in(0,1), R_{n}$ converges in distribution as $n \rightarrow \infty$ to $R$, the unique solution of the random equation (3.23). 
Moreover, the convergence does not depend on $R_{0}$. See Theorem 1.5 of Vervaat (1979) for more details.

Let $X^{\prime}$ be independent of $(X, Y)$ and have the same distribution of $X$. We have

$$
\begin{aligned}
\mathbb{P}\left(Y\left(X+X^{\prime}\right)>x\right) & =\int_{0}^{1} \mathbb{P}\left(X+X^{\prime}>\frac{x}{y}\right) \mathbb{P}(Y \in \mathrm{d} y) \\
& \sim 2 \int_{0}^{1} \bar{F}\left(\frac{x}{y}\right) \mathbb{P}(Y \in \mathrm{d} y) \\
& =o(1) \bar{F}(x),
\end{aligned}
$$

where we used $F \in \mathcal{S}$ in the second step and $F \in \mathcal{R}_{-\infty}$ and $\theta_{1}>0$ in the last step. Thus, there exists some $x_{0}>0$ large enough such that for all $x>x_{0}$,

$$
\mathbb{P}\left(Y\left(X+X^{\prime}\right)>x\right) \leq \bar{F}(x)
$$

Letting $R_{0}=X^{\prime} \mid X^{\prime}>x_{0}$, we claim that

$$
Y\left(X+R_{0}\right) \stackrel{d}{\leq} R_{0}
$$

Actually, when $x \leq x_{0}$, it is clear that $\mathbb{P}\left(Y\left(X+R_{0}\right)>x\right) \leq 1=\mathbb{P}\left(R_{0}>x\right)$. When $x \leq x_{0}$, by $(3.25)$ we have

$$
\begin{aligned}
\mathbb{P}\left(Y\left(X+R_{0}\right)>x\right) & =\frac{\mathbb{P}\left(Y\left(X+X^{\prime}\right)>x, X^{\prime}>x_{0}\right)}{\mathbb{P}\left(X^{\prime}>x_{0}\right)} \\
& \leq \frac{\mathbb{P}\left(Y\left(X+X^{\prime}\right)>x\right)}{\mathbb{P}\left(X^{\prime}>x_{0}\right)} \\
& \leq \frac{\bar{F}(x)}{\mathbb{P}\left(X^{\prime}>x_{0}\right)} \\
& =\mathbb{P}\left(R_{0}>x\right)
\end{aligned}
$$

Hence, relation (3.26) holds for each case. From (3.26) it is clear that

$$
R_{n} \stackrel{d}{\leq} R_{n-1}, \quad n=1,2, \ldots
$$


Therefore,

$$
R \stackrel{d}{\leq} R_{n} \stackrel{d}{=} R_{0} \prod_{i=1}^{n} Y_{i}+\sum_{k=1}^{n} X_{k} \prod_{i=1}^{k} Y_{i}, \quad n=0,1,2, \ldots
$$

When $n=2$, the above random inequality gives that, for all $x$,

$$
\begin{aligned}
\mathbb{P}(R>x) & \leq \mathbb{P}\left(R_{0} Y_{1} Y_{2}+X_{1} Y_{1}+X_{2} Y_{1} Y_{2}>x\right) \\
& =\int_{0}^{1} \mathbb{P}\left(R_{0} Y_{2}+X_{1}+X_{2} Y_{2}>\frac{x}{y}\right) \mathbb{P}\left(Y_{1} \in \mathrm{d} y\right) .
\end{aligned}
$$

By Lemma 2.24(ii),

$$
\begin{aligned}
\mathbb{P}\left(R_{0} Y_{2}+X_{2} Y_{2}>x\right) & =\int_{0}^{1} \mathbb{P}\left(R_{0}+X_{2}>\frac{x}{y}\right) \mathbb{P}\left(Y_{2} \in \mathrm{d} y\right) \\
& \sim\left(\frac{1}{\bar{F}\left(x_{0}\right)}+1\right) \int_{0}^{1} \bar{F}\left(\frac{x}{y}\right) \mathbb{P}\left(Y_{2} \in \mathrm{d} y\right) \\
& =o(1) \bar{F}(x)
\end{aligned}
$$

Then, applying Lemma 2.24(ii) again to (3.27) yields that

$$
\mathbb{P}(R>x) \lesssim \int_{0}^{1} \bar{F}\left(\frac{x}{y}\right) \mathbb{P}\left(Y_{1} \in \mathrm{d} y\right)=\mathbb{P}\left(X_{1} Y_{1}>x\right)
$$

A combination of (3.24) and (3.28) gives that

$$
\mathbb{P}(R>x) \sim \mathbb{P}\left(X_{1} Y_{1}>x\right)
$$

It remains to prove that

$$
\int_{0}^{\infty} \bar{F}\left(x \mathrm{e}^{r s}\right) \mathrm{d} \lambda_{s} \sim \mathbb{P}\left(X_{1} Y_{1}>x\right)
$$

Since $Y_{1} \in(0,1)$, it is easy to verify that the distribution of $X_{1} Y_{1}$ still belongs to the class $\mathcal{R}_{-\infty}$. By relation $(2.19)$, for arbitrarily chosen $p>0$ there are $x_{0}>0$ and $c>0$ such that, uniformly for all $k=1,2, \ldots$ and $x \geq x_{0}$,

$$
\frac{1}{\mathbb{P}\left(X_{1} Y_{1}>x\right)} \mathbb{P}\left(X_{k} \prod_{i=1}^{k} Y_{i}>x\right) \leq c \mathbb{E}\left(\prod_{i=2}^{k} Y_{i}\right)^{p}=c\left(\mathbb{E} Y_{1}^{p}\right)^{k-1}
$$


Therefore, by the dominated convergence theorem,

$$
\begin{aligned}
\lim _{x \rightarrow \infty} \frac{\int_{0}^{\infty} \bar{F}\left(x \mathrm{e}^{r s}\right) \mathrm{d} \lambda_{s}}{\mathbb{P}\left(X_{1} Y_{1}>x\right)} & =\lim _{x \rightarrow \infty} \frac{\sum_{k=1}^{\infty} \mathbb{P}\left(X_{k} \prod_{i=1}^{k} Y_{i}>x\right)}{\mathbb{P}\left(X_{1} Y_{1}>x\right)} \\
& =1+\sum_{k=2}^{\infty} \lim _{x \rightarrow \infty} \frac{\mathbb{P}\left(X_{k} \prod_{i=1}^{k} Y_{i}>x\right)}{\mathbb{P}\left(X_{1} Y_{1}>x\right)} \\
& =1 .
\end{aligned}
$$

From (3.29) and (3.30) we obtain relation (3.21).

Proof of Theorem 3.5. The proof can be given by copying the proof of Theorem

3.4 with the only modification that we use Lemma 3.15 instead of Lemma 3.14.

\subsubsection{Proof of Theorem 3.6}

Konstantinides et al. (2002) investigated the asymptotic behavior of the ruin probability of the compound Poisson model. In their model, the surplus process is expressed as

$$
S_{r}(t)=x \mathrm{e}^{r t}+p \int_{0}^{t} \mathrm{e}^{r(t-s)} \mathrm{d} s-\sum_{k=1}^{\infty} X_{k} \mathrm{e}^{r\left(t-\tau_{k}\right)} 1_{\left(\tau_{k} \leq t\right)}, \quad t \geq 0,
$$

where $x \geq 0$ is the initial surplus, $p>0$ is the constant premium rate, and $\left\{X_{k}, k=\right.$ $1,2, \ldots\},\left\{\tau_{k}, k=1,2, \ldots\right\}$, and $r$ are the same as appearing in relation (3.3). The counting process $\left(N_{t}\right)_{t \geq 0}$ generated by $\left\{\tau_{k}, k=1,2, \ldots\right\}$ is a Poisson process with intensity $\lambda>0$. The ruin probability is defined as

$$
\psi_{r}(x)=\mathbb{P}\left(\inf _{0<t<\infty} S_{r}(t)<0\right) .
$$

Theorem 2.1 of Konstantinides et al. (2002) shows that, if $F_{e} \in \mathcal{A}$, then

$$
\psi_{r}(x) \sim \frac{\lambda}{r} \int_{x}^{\infty} \frac{\bar{F}(y)}{y} \mathrm{~d} y .
$$


Based on relation (3.31) we produce the following result:

Lemma 3.16. Consider the discounted aggregate claims described in relation (3.3), in which $\left(N_{t}\right)_{t \geq 0}$ is a Poisson process with intensity $\lambda>0$. If $F_{e} \in \mathcal{A}$, then

$$
\mathbb{P}\left(D_{r}(\infty)>x\right) \sim \frac{\lambda}{r} \int_{x}^{\infty} \frac{\bar{F}(y)}{y} \mathrm{~d} y
$$

Proof. In terms of the model of Konstantinides et al. (2002),

$$
\psi_{r}(x)=\mathbb{P}\left(\sup _{0<t<\infty}\left(D_{r}(t)-p \int_{0}^{t} \mathrm{e}^{-r s} \mathrm{~d} s\right)>x\right)
$$

It follows that

$$
\psi_{r}(x) \leq \mathbb{P}\left(D_{r}(\infty)>x\right) \leq \psi_{r}(x-p / r)
$$

By (3.31) and integration by parts,

$$
\psi_{r}(x) \sim \frac{\mu \lambda}{r}\left(\frac{\overline{F_{e}}(x)}{x}-\int_{x}^{\infty} \frac{\overline{F_{e}}(y)}{y^{2}} \mathrm{~d} y\right)=\frac{\mu \lambda}{r}\left(K_{11}(x)-K_{12}(x)\right)
$$

Changing $x$ into $x-p / r$ in the above yields that

$$
\psi_{r}(x-p / r) \sim \frac{\mu \lambda}{r}\left(\frac{\overline{F_{e}}(x-p / r)}{x-p / r}-\int_{x-p / r}^{\infty} \frac{\overline{F_{e}}(y)}{y^{2}} \mathrm{~d} y\right)=\frac{\mu \lambda}{r}\left(K_{21}(x)-K_{22}(x)\right)
$$

Since $F_{e} \in \mathcal{A} \subset \mathcal{L}$

$$
K_{11}(x) \sim K_{21}(x), \quad K_{12}(x) \sim K_{22}(x)
$$

In order to infer $\psi_{r}(x) \sim \psi_{r}(x-p / r)$, it suffices to show that

$$
\limsup _{x \rightarrow \infty} \frac{K_{12}(x)}{K_{11}(x)}<1
$$


Since $F_{e} \in \mathcal{A}$, there exist some $v$ and $\varepsilon, v>1$ and $0<\varepsilon<1$, such that $\overline{F_{e}}(v x) / \overline{F_{e}}(x) \leq$ $1-\varepsilon$ holds for all large $x$. Hence, for all large $x$,

$$
\begin{aligned}
\frac{K_{12}(x)}{K_{11}(x)} & =\sum_{n=1}^{\infty} \int_{x v^{n-1}}^{x v^{n}} \frac{\overline{F_{e}}(y)}{\overline{F_{e}}(x)} \frac{x}{y^{2}} \mathrm{~d} y \leq \sum_{n=1}^{\infty} \int_{x v^{n-1}}^{x v^{n}} \frac{\overline{F_{e}}\left(x v^{n-1}\right)}{\overline{F_{e}}(x)} \frac{x}{y^{2}} \mathrm{~d} y \\
& \leq \sum_{n=1}^{\infty}(1-\varepsilon)^{n-1} \int_{x v^{n-1}}^{x v^{n}} \frac{x}{y^{2}} \mathrm{~d} y=\frac{v-1}{v-1+\varepsilon} .
\end{aligned}
$$

This proves (3.34). Therefore by (3.31) and (3.33), relation (3.32) follows immediately.

Lemma 3.17. For a distribution $F$ on $[0, \infty)$ with a finite positive expectation, if relation (2.12) with $F$ replaced by $F_{e}$ holds for some $v>1$, then

$$
\lim _{t \rightarrow \infty} \limsup _{x \rightarrow \infty} \frac{\int_{t}^{\infty} \bar{F}\left(x \mathrm{e}^{r s}\right) \mathrm{d} s}{\int_{0}^{\infty} \bar{F}\left(x \mathrm{e}^{r s}\right) \mathrm{d} s}=0 .
$$

Proof. Clearly,

$$
\frac{\int_{t}^{\infty} \bar{F}\left(x \mathrm{e}^{r s}\right) \mathrm{d} s}{\int_{0}^{\infty} \bar{F}\left(x \mathrm{e}^{r s}\right) \mathrm{d} s}=\frac{-\int_{x \mathrm{e}^{r t}}^{\infty} \frac{1}{y} \mathrm{~d} \overline{F_{e}}(y)}{-\int_{x}^{\infty} \frac{1}{y} \mathrm{~d} \overline{F_{e}}(y)}=\frac{\frac{\overline{F_{e}}\left(x \mathrm{e}^{r t}\right)}{x \mathrm{e}^{r t}}-\int_{x \mathrm{e}^{r t}}^{\infty} \frac{\overline{F_{e}}(y)}{y^{2}} \mathrm{~d} y}{\frac{\overline{F_{e}}(x)}{x}-\int_{x}^{\infty} \frac{\overline{F_{e}}(y)}{y^{2}} \mathrm{~d} y} .
$$

By (3.34), there is some constant $c^{*}>0$ such that, uniformly for all $t>0$,

$$
\frac{\int_{t}^{\infty} \bar{F}\left(x \mathrm{e}^{r s}\right) \mathrm{d} s}{\int_{0}^{\infty} \bar{F}\left(x \mathrm{e}^{r s}\right) \mathrm{d} s} \leq c^{*} \frac{\overline{F_{e}}\left(x \mathrm{e}^{r t}\right)}{\frac{x \mathrm{e}^{r t}}{\overline{F_{e}}(x)}} \leq c^{*} \mathrm{e}^{-r t} .
$$

Therefore, (3.35) holds.

Proof of Theorem 3.6. The proof can be given by copying the proof of Theorem 3.4 with the only modification that we use Lemmas 3.16 and 3.17 instead of Lemmas 3.10 and 3.14 . 


\subsection{Simulation}

In this section, we simulate the uniform convergence in (3.5) on a finite time interval $(0, T]$. Set $T=60$, i.e., the maximum horizon we consider is 60 years. Assume that the constant force of interest is $r=0.1$, claims arrive according to a Poisson process with intensity $\lambda=5$, and the claim sizes are i.i.d. with common Pareto distribution $F$ satisfying $\bar{F}(x)=1 /(1+x)^{2}$ for $x \geq 0$. It is obvious that $F \in \mathcal{S}$ and $F_{e} \in \mathcal{A}$.

For each $x$, we find the supremum

$$
\sup _{t \in(0, T]}\left|\frac{\mathbb{P}\left(D_{r}(t)>x\right)}{\lambda \int_{0}^{t} \bar{F}\left(x \mathrm{e}^{r s}\right) \mathrm{d} s}-1\right|
$$

Then we let $x$ increase to see whether, as predicted by relation (3.5), the above supremum goes to 0 and how fast the convergence is. We execute our simulation in $\mathrm{R}$ software. The simulation result is shown in Figure 3.1 below.

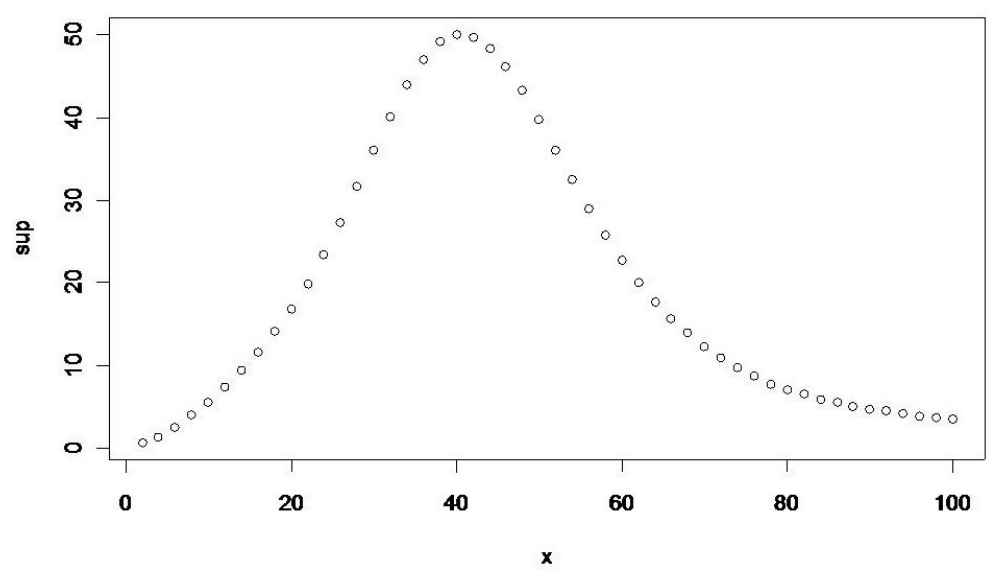

Figure 3.1: Uniform convergence on $(0, T]$ with Pareto $F$ 
From Figure 3.1 we see that as $x$ increases the supremum given in (3.36) converges to 0 . The convergence speed is reasonable seeing that the expected claim size is 1 . Indeed, the supremum is very close to 0 when $x$ comes up to 100 . 


\section{CHAPTER 4 \\ THE MAXIMUM EXCEEDANCE OVER A RANDOM WALK}

In this chapter, we consider a problem in the field of probability. Motivated by the observations that many problems in applied fields, including corporate finance, insurance risk, and production systems, can be reduced to the study of the distribution of the maximum exceedance of a sequence of random variables over a renewal threshold, we derive a unified asymptotic formula for the tail probability of such a maximum exceedance for both light-tailed and heavy-tailed cases. An application of the main result to corporate finance is proposed in Section 4.2. The main result will play an important role in the proofs of two light-tailed cases in Chapter 5. This chapter is based on the joint research paper Hao et al. (2009).

\subsection{Introduction and Main Result}

Let $\left\{Y_{n}, n=1,2, \ldots\right\}$ be a sequence of i.i.d. random variables with generic random variable $Y$, common distribution $F$ on $(-\infty, \infty)$, and $0<\nu_{F}=\int_{0}^{\infty} \bar{F}(y) \mathrm{d} y<$ $\infty$. For every constant $\mu>0$, the maximum

$$
M_{0}=\sup _{n \geq 1}\left(Y_{n}-(n-1) \mu\right)
$$

is finite almost surely. If the equilibrium distribution $F_{e}$ is long tailed, then it is easy to check that

$$
\lim _{x \rightarrow \infty} \frac{\mathbb{P}\left(M_{0}>x\right)}{\int_{x}^{\infty} \bar{F}(y) \mathrm{d} y}=\frac{1}{\mu}
$$


Actually, on the one hand,

$$
\mathbb{P}\left(M_{0}>x\right) \leq \sum_{n=1}^{\infty} \bar{F}(x+(n-1) \mu) \leq \frac{1}{\mu} \int_{x-\mu}^{\infty} \bar{F}(y) \mathrm{d} y \sim \frac{1}{\mu} \int_{x}^{\infty} \bar{F}(y) \mathrm{d} y .
$$

On the other hand,

$$
\begin{aligned}
\mathbb{P}\left(M_{0}>x\right) & \geq \sum_{n=1}^{\infty} \bar{F}(x+(n-1) \mu)-\sum_{1 \leq n<m<\infty} \bar{F}(x+(n-1) \mu) \bar{F}(x+(m-1) \mu) \\
& \geq \sum_{n=1}^{\infty} \bar{F}(x+(n-1) \mu)-\left(\sum_{n=1}^{\infty} \bar{F}(x+(n-1) \mu)\right)^{2} \\
& \geq \frac{1}{\mu} \int_{x}^{\infty} \bar{F}(y) \mathrm{d} y-\left(\frac{1}{\mu} \int_{x-\mu}^{\infty} \bar{F}(y) \mathrm{d} y\right)^{2} \\
& \sim \frac{1}{\mu} \int_{x}^{\infty} \bar{F}(y) \mathrm{d} y .
\end{aligned}
$$

Hence, relation (4.1) holds.

Motivated by the observation above, in this chapter we study the tail probability of the maximum exceedance of the sequence $\left\{Y_{n}, n=1,2, \ldots\right\}$ over a random walk with positive drift. Precisely, let $\left\{\left(X_{n}, Y_{n}\right), n=1,2, \ldots\right\}$ be a sequence of i.i.d. random pairs with generic random pair $(X, Y)$. Assume that $\mathbb{E} X=\mu>0$ and that $Y$ follows a distribution $F$ on $(-\infty, \infty)$. Then, the maximum

$$
M=\sup _{n \geq 1}\left(Y_{n}-S_{n-1}\right)
$$

with $S_{n-1}=\sum_{i=1}^{n-1} X_{i}$, is finite almost surely.

For the sake of consistency, for a random variable $X$ with mean $\mu>0$ we make a convention that

$$
\left.\frac{\alpha}{1-\mathbb{E} \mathrm{e}^{-\alpha X}}\right|_{\alpha=0}=\frac{1}{\mu}
$$

The main result of this chapter is given below: 
Theorem 4.1. Consider the i.i.d. sequence $\left\{\left(X_{n}, Y_{n}\right), n=1,2, \ldots\right\}$ and the maximum $M$ defined in (4.2), where $\mathbb{E} X=\mu>0$ and $Y$ is distributed by $F$. Then, the relation

$$
\lim _{x \rightarrow \infty} \frac{\mathbb{P}(M>x)}{\int_{x}^{\infty} \bar{F}(y) \mathrm{d} y}=\frac{\alpha}{1-\mathbb{E}^{-\alpha X}}
$$

holds under one of the following groups of conditions:

(i) $F_{e} \in \mathcal{L}(\alpha)$ for some $\alpha \geq 0, \mathbb{E} X^{2}<\infty$, and $\mathbb{E}^{-\beta X}<1$ for some $\beta>\alpha$;

(ii) $F_{e} \in \mathcal{S}(\alpha)$ for some $\alpha \geq 0, \mathbb{P}(-X>x)=o(\bar{F}(x))$, and $\mathbb{E} \mathrm{e}^{-\alpha X}<1$ provided $\alpha>0$.

Clearly, $\mathbb{E} \mathrm{e}^{-\gamma X}$, as a function of $\gamma$, is convex over all $\gamma$ for which $\mathbb{E}^{-\gamma X}$ is finite. Hence for case (i), $\mathbb{E} \mathrm{e}^{-\gamma X}<1$ for every $\gamma \in(0, \beta]$.

As shown in Lemma 2.22, for every $\alpha \geq 0$, the condition $F_{e} \in \mathcal{L}(\alpha)$ is equivalent to relation $(2.22)$, i.e.,

$$
\lim _{x \rightarrow \infty} \frac{\bar{F}(x)}{\int_{x}^{\infty} \bar{F}(y) \mathrm{d} y}=\alpha .
$$

In particular, the condition $F_{e} \in \mathcal{L}$ (or, equivalently, relation $(2.22)$ with $\alpha=0$ ) is fulfilled by most cited heavy-tailed distributions including all long-tailed or dominatedlyvarying-tailed distributions with finite mean; see Lemma 2.20.

It is worth mentioning that Theorem 4.1 allows $X$ and $Y$ to be arbitrarily dependent. However, the asymptotic relation for $\mathbb{P}(M>x)$ completely eliminates impact of the dependence of $(X, Y)$.

Some closely related works are summarized as follows:

(i) Robert (2005) considered a special case of our Theorem 4.1(i) with $\alpha=0$ and $X$ positive and proposed an application to ruin theory in the presence of dividends 
paid out at a sequence of random epochs. He considered the renewal risk model for an insurance company. Assume that the insurance company uses a stopping time $\tau_{1}$ to decide when the surplus $S_{\tau_{1}}>0$ is sufficiently large that a part $f\left(S_{\tau_{1}}\right)$ is distributed to the shareholders and the other part $S_{\tau_{1}}-f\left(S_{\tau_{1}}\right)$ kept to reinforce the solvency margin. At this time, the surplus reduces to 0 . The same rule is then used to define $\tau_{2}$. In this way, we obtain a sequence of stopping times $\left\{\tau_{n}, n=1,2, \ldots\right\}$ and a sequence of dividends $\left\{f\left(S_{\tau_{n}}-S_{\tau_{n-1}}\right), n=1,2, \ldots\right\}$, where $\tau_{0}=0$. If we set $X_{n}=S_{\tau_{n}}-S_{\tau_{n-1}}-f\left(S_{\tau_{n}}-S_{\tau_{n-1}}\right)$ and $Y_{n}=\sup _{\tau_{n-1} \leq t<\tau_{n}}\left(S_{\tau_{n-1}}-S_{t}\right)$, then the ruin probability, i.e., the probability that the surplus process $S$ goes below 0 sometime is $\psi(x)=\mathbb{P}(M>x)$, where $M$ is defined in (4.2). According to Theorem 4.1(i), we can give an explicit asymptotic expression for the ruin probability $\psi(x)$ as

$$
\psi(x) \sim \frac{1}{\mathbb{E} X_{1}} \int_{x}^{\infty} \mathbb{P}(Y>y) \mathrm{d} y .
$$

(ii) Araman and Glynn (2006) systematically studied the same problem in the framework of a perturbed random walk for various cases. Their Theorem 3 corresponds to a special case of our Theorem 4.1(i) with $\alpha>0, X, Y$ independent, and $F$ exponential, but under the condition $\mathbb{E}^{-\alpha X}<1$, which is slightly weaker than our condition $\mathbb{E} \mathrm{e}^{-\beta X}<1$ for some $\beta>\alpha$. Their Theorem 4 , assuming that $F$ has a continuous hazard rate function converging to 0 , corresponds to a special case of our Theorem 4.1(i) with $\alpha=0$ and $X, Y$ independent. Indeed, by L'Hôspital's rule the assumption that $F$ has a continuous hazard rate function converging to 0 implies that $F_{e} \in \mathcal{L}(0)$.

(iii) Palmowski and Zwart (2007) also studied the same problem but in the 
framework of a regenerative process. In terms of their model in which the regenerative process $S=\left(S_{t}\right)_{t \geq 0}$ has renewal epochs $0=T_{0}<T_{1}<\cdots$, the random variables $X_{n}$ and $Y_{n}$ in our theorems correspond to $S_{T_{n-1}}-S_{T_{n}}$ and $\sup _{T_{n-1} \leq t<T_{n}} S_{t}-S_{T_{n-1}}$, respectively. In particular, their Theorem 1 corresponds to our Theorem 4.1(ii) with $\alpha=0$ under the assumption that the equilibrium distribution of $(-X) \vee Y$ is subexponential and their Theorem 2 corresponds to our Theorem 4.1 with $\alpha>0$ under the following three assumptions: (1) $F \in \mathcal{L}(\alpha)$, (2) $\mathbb{E} \mathrm{e}^{-\alpha X}<1$, and (3) $\mathbb{P}(Y-\tilde{X}>x) \sim \mathbb{P}(Y>x) \mathbb{E} \mathrm{e}^{-\alpha \widetilde{X}}$ with $\widetilde{X}$ identically distributed as $X$ and independent of $(X, Y)$. We need to point out that the assumptions they used in their Theorem 2 are slightly more general than ours.

The rest of this chapter is organized as follows: Section 4.2 proposes an application to corporate finance, Section 4.3 prepares several lemmas, Sections 4.4 and 4.5 respectively prove cases (i) and (ii) of Theorem 4.1, and at last Section 4.6 gives two simulation results.

\subsection{Application to Corporate Finance}

Consider an incorporated firm whose profit during the $n$th fiscal year is denoted by $Z_{n}, n=1,2, \ldots$. At the end of each fiscal year, the firm will pay out to shareholders a part of its profit as dividend if it earns money in that year; otherwise, it will issue new equity to raise money. More precisely, introduce two constants $\Delta$ and $\epsilon, 0<\Delta<1$ and $\epsilon>0$, such that the amount $\Delta Z_{n}$ will be paid out if $Z_{n}>0$ and the amount $-\epsilon Z_{n}$ will be raised otherwise. We assume that at the end of each fiscal year the firm 
liquidates its capital and restarts operation in the very beginning of the next fiscal year. For each real number $a$, write $a^{+}=a \vee 0$ and $a^{-}=-(a \wedge 0)$. Then, the increment of capital amount during the $n$th fiscal year after liquidation will be

$$
X_{n}=Z_{n}-\Delta Z_{n}^{+}+\epsilon Z_{n}^{-}, \quad n=1,2, \ldots
$$

and the capital amount up to the end of the $n$th fiscal year before liquidation will be

$$
R_{n}=x+\sum_{i=1}^{n-1} X_{i}+Z_{n}, \quad n=1,2, \ldots,
$$

with $R_{0}=x>0$ being the initial capital of the firm.

We are interested in the probability of the so-called bankrupt event. Such an event describes that the financial situation of the firm becomes too bad to survive from budget deficits, or, in other words, the capital surplus of the firm goes below some critical level $b$. Denote this probability by $\psi(x, b)$, which, when $b=0$, is called the ruin probability in risk theory. Then,

$$
\psi(x, b)=\mathbb{P}\left(\inf _{n \geq 1} R_{n}<b \mid R_{0}=x\right) .
$$

In order to use Theorem 4.1 to derive an asymptotic estimate for $\psi(x, b)$, assume that $\left\{Z_{n}, n=1,2, \ldots\right\}$ is a sequence of i.i.d. random variables, implying that the operation of the firm in each year does not depend on its financial situation in the beginning of that year. Let $Y_{n}=-Z_{n}$ for $n=1,2, \ldots$ Therefore, $\left(X_{n}, Y_{n}\right), n=1,2, \ldots$, constitute a sequence of i.i.d. random pairs, and

$$
\psi(x, b)=\mathbb{P}\left\{\sup _{n \geq 1}\left(Y_{n}-\sum_{i=1}^{n-1} X_{i}\right)>x-b\right\}
$$


Denote by $Z, X$, and $Y$ the generic random variables for $Z_{n}, X_{n}$, and $Y_{n}$, respectively, and denote by $F$ the distribution of $Y$. Then under the conditions of Theorem 4.1, we have

$$
\lim _{x \rightarrow b \rightarrow \infty} \frac{\psi(x, b)}{\int_{x-b}^{\infty} \bar{F}(y) \mathrm{d} y}=\frac{\alpha}{1-\mathbb{E} \mathrm{e}^{-\alpha X}}
$$

To illustrate the conditions of Theorem 4.1(i), assume that $Z$ follows the distribution

$$
\mathbb{P}(Z \leq z)= \begin{cases}1-\frac{\alpha}{\tilde{\alpha}+\alpha} \mathrm{e}^{-\tilde{\alpha} z}, & z \geq 0, \\ \frac{\tilde{\alpha}}{\tilde{\alpha}+\alpha} \mathrm{e}^{\alpha z}, & z<0,\end{cases}
$$

for some $\alpha>\tilde{\alpha}>0$. Recall $X=Z-\Delta Z^{+}+\epsilon Z^{-}$and $Y=-Z$. Clearly, $F \in \mathcal{L}(\alpha)$ and $0<\int_{0}^{\infty} \bar{F}(y) \mathrm{d} y<\infty$. If $\epsilon \geq 1$, then all conditions of Theorem 4.1 are obviously satisfied. As for $0<\epsilon<1$, choose $\Delta$ and $\epsilon$ satisfying

$$
0<\Delta<1 \quad \text { and } \quad \frac{\tilde{\alpha}^{2}+\tilde{\alpha} \alpha(1-\Delta)}{\tilde{\alpha}^{2}+\tilde{\alpha} \alpha(1-\Delta)+\alpha^{2}(1-\Delta)}<\epsilon<1
$$

Then,

$$
\mathbb{E} X=\frac{(1-\Delta) \alpha^{2}-(1-\epsilon) \tilde{\alpha}^{2}}{\tilde{\alpha} \alpha(\tilde{\alpha}+\alpha)}>0,
$$

and for all $\beta \in\left(\alpha, \frac{(1-\Delta) \alpha^{2}-(1-\epsilon) \tilde{\gamma}^{2}}{(1-\Delta)(1-\epsilon)(\tilde{\gamma}+\alpha)}\right)$,

$$
\mathbb{E}^{-\beta X}=\frac{\tilde{\alpha} \alpha}{\tilde{\alpha}+\alpha}\left(\frac{1}{\tilde{\alpha}+\beta(1-\Delta)}+\frac{1}{\alpha-\beta(1-\epsilon)}\right)<1
$$

Therefor, all conditions of Theorem 4.1(i) are satisfied.

In sum, if $Z$ follows the distribution given in $(4.5)$, then for $(\Delta, \epsilon)$ belonging to $(0,1) \times[1, \infty)$ or satisfying $(4.6)$, by relation $(4.4)$ we have

$$
\lim _{x \rightarrow b \rightarrow \infty} \frac{\psi(x, b)}{\mathrm{e}^{-\alpha(x-b)}}=\frac{\tilde{\alpha}^{2} \epsilon+\alpha \tilde{\alpha}(1-\Delta) \epsilon}{\alpha^{2}(1-\Delta) \epsilon-\alpha \tilde{\alpha}(1-\Delta)(1-\epsilon)-\tilde{\alpha}^{2}(1-\epsilon)} .
$$


It is even easier to construct a distribution for the random variable $Z$ such that all conditions of Theorem 4.1(ii) are satisfied.

\subsection{Lemmas}

Lemma 4.2 (Lemma 3.1 of Robert (2005)). Let $\left\{\xi_{n}, n=1,2, \ldots\right\}$ be a sequence of i.i.d. random variables with generic random variable $\xi$ satisfying $-\infty<\mathbb{E} \xi<0$ and $\mathbb{P}(\xi>0)>0$. Then, $\mathbb{E}\left(\xi^{+}\right)^{2}<\infty$ if and only if

$$
\sum_{n=1}^{\infty} \mathbb{P}\left(\sum_{i=1}^{n} \xi_{i}>0\right)<\infty
$$

Lemma 4.3. Let $\left\{\xi_{n}, n=1,2, \ldots\right\}$ be a sequence of i.i.d. random variables with finite mean $\mu$. Then for arbitrarily small $\varepsilon, \delta>0$, there exists some constant $c>0$ such that

$$
\mathbb{P}\left(\bigcap_{n=1}^{\infty}\left(n(\mu-\delta)-c \leq \sum_{i=1}^{n} \xi_{i} \leq n(\mu+\delta)+c\right)\right)>1-\varepsilon
$$

Proof. Follow the proof of Lemma 3.1 of Asmussen et al. (1999) with some obvious modifications.

Lemma 4.4 (Theorem 2 of Veraverbeke (1977)). Let $\left\{\xi_{n}, n=1,2, \ldots\right\}$ be a sequence of i.i.d. random variables with common distribution $F$ and finite mean $\mu$. Let $-\alpha$ be the left abscissa of convergence of $f(\lambda)=\int_{-\infty}^{\infty} \mathrm{e}^{-\lambda x} F(\mathrm{~d} x)$. Denote by $W(\cdot)$ the distribution of $\sup _{n \geq 0} \sum_{i=1}^{n} \xi_{i}$.

(i) Suppose $\alpha>0$. If $f(-\alpha)<1$ then $\bar{W}(x)=o\left(\mathrm{e}^{-\alpha x}\right)$ and $F \in \mathcal{S}(\alpha) \Longleftrightarrow$ $W \in \mathcal{S}(\alpha)$ each of which implies that

$$
\lim _{x \rightarrow \infty} \frac{\bar{W}(x)}{\bar{F}(x)}=c(\alpha)
$$


holds for some constant $c(\alpha)>0$.

(ii) Suppose $\alpha=0$. If $\mu<0$, then $F_{e} \in \mathcal{S} \Longleftrightarrow W \in \mathcal{S}$ each of which implies

$$
\lim _{x \rightarrow \infty} \frac{\bar{W}(x)}{\int_{x}^{\infty} \bar{F}(y) \mathrm{d} y}=-\frac{1}{\mu}
$$

Lemma 4.5. Assume $F_{e} \in \mathcal{S}(\alpha)$ for some $\alpha \geq 0$. Let $\left\{\xi_{n}, n=1,2, \ldots\right\}$ be a sequence of i.i.d. random variables with generic random variable $\xi$ satisfying $-\infty<\mathbb{E} \xi<0$, $\mathbb{P}(\xi>x)=o(\bar{F}(x))$, and $\mathbb{E} \mathrm{e}^{\alpha \xi}<1$ provided $\alpha>0$. Then,

$$
\mathbb{P}\left(\sup _{n \geq 0} \sum_{i=1}^{n} \xi_{i}>x\right)=o\left(\overline{F_{e}}(x)\right)
$$

Proof. For arbitrarily fixed $N$, we have

$$
\begin{aligned}
\mathbb{P}\left(\sup _{n \geq 0} \sum_{i=1}^{n} \xi_{i}>x\right) & \leq \mathbb{P}\left(\sup _{0 \leq n \leq N} \sum_{i=1}^{n} \xi_{i}>x\right)+\mathbb{P}\left(\sum_{i=1}^{N} \xi_{i}+\sup _{n \geq N} \sum_{i=N+1}^{n} \xi_{i}>x\right) \\
& =I_{1}(x, N)+I_{2}(x, N) .
\end{aligned}
$$

By Lemma 2.22, $\mathbb{P}(\xi>x)=o(\bar{F}(x))=o\left(\overline{F_{e}}(x)\right)$. Then by Lemma 2.24(ii),

$$
I_{1}(x, N) \leq \mathbb{P}\left(\sum_{i=1}^{N}\left(\xi_{i} \vee 0\right)>x\right)=o\left(\overline{F_{e}}(x)\right)
$$

To consider $I_{2}(x, N)$, for arbitrarily small $\varepsilon>0$, introduce a random variable $\eta$ satisfying

$$
\mathbb{P}(\eta>x)=\mathbb{P}(\xi>x) \vee \varepsilon \bar{F}(x)
$$

Clearly, $\mathbb{P}(\eta>x) \sim \varepsilon \bar{F}(x)$. Since $\eta=\eta(\varepsilon)$ converges to $\xi$ in distribution as $\varepsilon \searrow 0$, for all small $\varepsilon>0$ we have $\mathbb{E} \eta<0$ and $\mathbb{E} \mathrm{e}^{\alpha \eta}<1$ provided $\alpha>0$. Let $\left\{\eta_{n}, n=1,2, \ldots\right\}$ be a sequence of i.i.d. copies of $\eta$ independent of $\left\{\xi_{n}, n=1,2, \ldots\right\}$. By Lemma 4.4, it holds for some constant $c(\alpha, \varepsilon)>0$ that

$$
\mathbb{P}\left(\sup _{n \geq N} \sum_{i=N+1}^{n} \eta_{i}>x\right)=\mathbb{P}\left(\sup _{n \geq 0} \sum_{i=1}^{n} \eta_{i}>x\right) \sim c(\alpha, \varepsilon) \overline{F_{e}}(x) .
$$


When $\alpha>0$, the expression of $c(\alpha, \varepsilon)$ is rather involved. However, when $\alpha=0$, we have the transparent expression $c(0, \varepsilon)=-\varepsilon \nu_{F} / \mathbb{E} \eta$. Then by Lemma 2.24(ii),

$$
I_{2}(x, N) \leq \mathbb{P}\left(\sum_{i=1}^{N} \xi_{i}+\sup _{n \geq N} \sum_{i=N+1}^{n} \eta_{i}>x\right) \sim\left(\mathbb{E} \mathrm{e}^{\alpha \xi}\right)^{N} c(\alpha, \varepsilon) \overline{F_{e}}(x)
$$

Plugging (4.9) and (4.10) into (4.8) yields that

$$
\limsup _{x \rightarrow \infty} \frac{1}{\overline{F_{e}}(x)} \mathbb{P}\left(\sup _{n \geq 0} \sum_{i=1}^{n} \xi_{i}>x\right) \leq\left(\mathbb{E} \mathrm{e}^{\alpha \xi}\right)^{N} c(\alpha, \varepsilon)
$$

If $\alpha>0$ with $\varepsilon$ fixed we let $N \rightarrow \infty$, while if $\alpha=0$ we let $\varepsilon \searrow 0$. Thus, in any case, the right-hand side of the above goes to 0 and the proof is complete.

\subsection{Proof of Theorem 4.1(i)}

\subsubsection{Preliminary Results}

Proposition 4.6. Under the conditions of Theorem 4.1(i), it holds for arbitrarily small $\varepsilon>0$, all $0<\delta<1$, and all large $k$ that

$$
\sum_{n=k+1}^{\infty} \mathbb{P}\left(Y_{n}-S_{n-1}>x, S_{n-1}<(n-1) \mu(1-\delta)\right) \lesssim \varepsilon \int_{x}^{\infty} \bar{F}(y) \mathrm{d} y
$$

Proof. Let $0<\delta<1$ and $D>0$ be arbitrarily fixed. For all $x>D$, according to the range of $S_{n-1}$ we split the left-hand side of (4.11) into three parts as

$$
\begin{aligned}
& \sum_{n=k+1}^{\infty}\left\{\mathbb{P}\left(Y_{n}-S_{n-1}>x, S_{n-1} \in(0,(n-1) \mu(1-\delta))\right)\right. \\
& \quad+\mathbb{P}\left(Y_{n}-S_{n-1}>x, S_{n-1} \in(-x+D, 0]\right) \\
& \left.\quad+\mathbb{P}\left(Y_{n}-S_{n-1}>x, S_{n-1} \in(-\infty,-x+D]\right)\right\} \\
& =J_{1}(x, k, \delta)+J_{2}(x, k, D)+J_{3}(x, k, D) .
\end{aligned}
$$


Using Lemmas 2.22 and 4.2, for arbitrarily small $\varepsilon>0$ and all large $k$,

$$
J_{1}(x, k, \delta) \leq \bar{F}(x) \sum_{n=k+1}^{\infty} \mathbb{P}\left(\sum_{i=1}^{n-1}\left(\mu(1-\delta)-X_{i}\right)>0\right) \lesssim \frac{\varepsilon}{2} \int_{x}^{\infty} \bar{F}(y) \mathrm{d} y
$$

Furthermore, by Lemma 2.23(i), there exist some constants $c_{0}, D>0$ such that for all $x \geq x+y-1 \geq D-1$ and all large $k$,

$$
\begin{aligned}
J_{2}(x, k, D) & =\sum_{n=k+1}^{\infty} \int_{-x+D}^{0} \bar{F}(x+y) \mathbb{P}\left(S_{n-1} \in \mathrm{d} y\right) \\
& \leq \sum_{n=k+1}^{\infty} \int_{-x+D}^{0}\left(\int_{x+y-1}^{x+y} \bar{F}(u) \mathrm{d} u\right) \mathbb{P}\left(S_{n-1} \in \mathrm{d} y\right) \\
& \leq \int_{x}^{\infty} \bar{F}(y) \mathrm{d} y \sum_{n=k+1}^{\infty} \int_{-x+D}^{0} \frac{\overline{F_{e}}(x+y-1)}{\overline{F_{e}}(x)} \mathbb{P}\left(S_{n-1} \in \mathrm{d} y\right) \\
& \leq c_{0} \int_{x}^{\infty} \bar{F}(y) \mathrm{d} y \sum_{n=k+1}^{\infty} \mathbb{E} \mathrm{e}^{-\beta\left(S_{n-1}-1\right)} \\
& \leq \frac{\varepsilon}{2} \int_{x}^{\infty} \bar{F}(y) \mathrm{d} y .
\end{aligned}
$$

For $D$ specified in (4.14) and for all $k$, employ Markov's inequality and Lemma 2.23(ii) to obtain that

$$
J_{3}(x, k, D) \leq \sum_{n=k+1}^{\infty} \mathbb{P}\left(S_{n-1} \leq-x+D\right) \leq \sum_{n=k+1}^{\infty} \frac{\mathbb{E} \mathrm{e}^{-\beta S_{n-1}}}{\mathrm{e}^{\beta(x-D)}}=o\left(\overline{F_{e}}(x)\right)
$$

Plugging (4.13)-(4.15) into (4.12) yields (4.11).

Proposition 4.7. Under the conditions of Theorem 4.1(i), it holds for each $k=$ $2,3, \ldots$ that

$$
\sum_{1 \leq n<m \leq k} \mathbb{P}\left(Y_{n}-S_{n-1}>x, Y_{m}-S_{m-1}>x\right)=o\left(\overline{F_{e}}(x)\right)
$$


Proof. Let $\delta>0$ be a constant satisfying $\beta(1-\delta)>\alpha$. For $1=n<m \leq k$,

$$
\begin{aligned}
& \mathbb{P}\left(Y_{1}>x, Y_{m}-S_{m-1}>x\right) \\
\leq & \mathbb{P}\left(-S_{m-1}>(1-\delta) x\right)+\mathbb{P}\left(Y_{1}>x, Y_{m}-S_{m-1}>x,-S_{m-1} \leq(1-\delta) x\right) \\
\leq & \mathrm{e}^{-\beta(1-\delta) x} \mathbb{E}^{-\beta S_{m-1}}+\mathbb{P}\left(Y_{1}>x, Y_{m}>\delta x\right) \\
= & o\left(\overline{F_{e}}(x)\right)
\end{aligned}
$$

where we used Markov's inequality and Lemmas 2.22 and 2.23(ii). Similarly, for $1<n<m \leq k$,

$$
\begin{aligned}
& \mathbb{P}\left(Y_{n}-S_{n-1}>x, Y_{m}-S_{m-1}>x\right) \\
\leq & \mathbb{P}\left(-S_{n-1}>(1-\delta) x\right)+\mathbb{P}\left(Y_{n}-S_{n-1}>x, Y_{m}-S_{m-1}>x,-S_{n-1} \leq(1-\delta) x\right) \\
\leq & \mathrm{e}^{-\beta(1-\delta) x} \mathbb{E} \mathrm{e}^{-\beta S_{n-1}} \\
& +\int_{-\infty}^{(1-\delta) x} \mathbb{P}\left(Y_{n}>x-y, Y_{m}-S_{n, m-1}>x-y\right) \mathbb{P}\left(-S_{n-1} \in \mathrm{d} y\right),
\end{aligned}
$$

where $S_{n, m-1}=\sum_{i=n}^{m-1} X_{i}$. By (4.17), it holds uniformly for all $y \leq(1-\delta) x$ that

$$
\mathbb{P}\left(Y_{n}>x-y, Y_{m}-S_{n, m-1}>x-y\right)=o(1) \overline{F_{e}}(x-y)
$$

Hence by Lemmas 2.23(ii) and 2.24(i),

$$
\begin{aligned}
& \mathbb{P}\left(Y_{n}-S_{n-1}>x, Y_{m}-S_{m-1}>x\right) \\
= & o\left(\overline{F_{e}}(x)\right)+o(1) \int_{-\infty}^{(1-\delta) x} \overline{F_{e}}(x-y) \mathbb{P}\left(-S_{n-1} \in \mathrm{d} y\right) \\
= & o\left(\overline{F_{e}}(x)\right) .
\end{aligned}
$$

A combination of (4.17) and (4.18) gives (4.16). 


\subsubsection{Proof of Theorem 4.1(i) for $\alpha>0$}

We first prove the asymptotic upper bound. For some $0<\delta<1$ and each $k=1,2, \ldots$,

$$
\begin{aligned}
\mathbb{P}(M>x) \leq & \left(\sum_{n=1}^{k}+\sum_{n=k+1}^{\infty}\right) \mathbb{P}\left(Y_{n}-S_{n-1}>x\right) \\
\leq & \sum_{n=1}^{k} \mathbb{P}\left(Y_{n}-S_{n-1}>x\right)+\sum_{n=k+1}^{\infty} \bar{F}(x+(n-1) \mu(1-\delta)) \\
& \quad+\sum_{n=k+1}^{\infty} \mathbb{P}\left(Y_{n}-S_{n-1}>x, S_{n-1}<(n-1) \mu(1-\delta)\right) \\
= & K_{1}(x, k)+K_{2}(x, k, \delta)+K_{3}(x, k, \delta) .
\end{aligned}
$$

By Proposition 4.6, it holds for arbitrarily small $\varepsilon>0$ and all large $k$ that

$$
K_{3}(x, k, \delta) \lesssim \frac{\varepsilon}{2} \int_{x}^{\infty} \bar{F}(y) \mathrm{d} y
$$

Since $F_{e} \in \mathcal{L}(\alpha)$, it holds for all large $k$ that

$$
K_{2}(x, k, \delta) \leq \frac{1}{\mu(1-\delta)} \int_{x+(k-1) \mu(1-\delta)}^{\infty} \bar{F}(y) \mathrm{d} y \lesssim \frac{\varepsilon}{2} \int_{x}^{\infty} \bar{F}(y) \mathrm{d} y .
$$

With $k$ specified in (4.20) and (4.21), by Lemma 2.24(i) and relation (2.22) we have

$$
K_{1}(x, k) \sim \bar{F}(x) \sum_{n=1}^{k} \mathbb{E} \mathrm{e}^{-\alpha S_{n-1}} \lesssim \frac{\alpha}{1-\mathbb{E} \mathrm{e}^{-\alpha X}} \int_{x}^{\infty} \bar{F}(y) \mathrm{d} y .
$$

Plugging (4.20)-(4.22) into (4.19) and using the arbitrariness of $\varepsilon>0$, we obtain that

$$
\limsup _{x \rightarrow \infty} \frac{\mathbb{P}(M>x)}{\int_{x}^{\infty} \bar{F}(u) \mathrm{d} u} \leq \frac{\alpha}{1-\mathbb{E e}^{-\alpha X}} .
$$

Next, we turn to prove the asymptotic lower bound. Obviously, for each $k=1,2, \ldots$, using Bonferroni's inequality,

$$
\begin{aligned}
\mathbb{P}(M>x) & \geq \mathbb{P}\left(\bigcup_{n=1}^{k}\left(Y_{n}-S_{n-1}>x\right)\right) \\
& \geq K_{1}(x, k)-\sum_{1 \leq n<m \leq k} \mathbb{P}\left(Y_{n}-S_{n-1}>x, Y_{m}-S_{m-1}>x\right),
\end{aligned}
$$


where $K_{1}(x, k)$ is the same as in (4.19). Similar to (4.22), for arbitrarily small $\varepsilon>0$ and all large $k$,

$$
K_{1}(x, k) \gtrsim(1-\varepsilon) \frac{\alpha}{1-\mathbb{E} \mathrm{e}^{-\alpha X}} \int_{x}^{\infty} \bar{F}(u) \mathrm{d} u
$$

By Proposition 4.7, relation (4.16) holds. Plugging (4.24) and (4.16) into (4.23) and using the arbitrariness of $\varepsilon>0$, we have

$$
\liminf _{x \rightarrow \infty} \frac{\mathbb{P}(M>x)}{\int_{x}^{\infty} \bar{F}(y) \mathrm{d} y} \geq \frac{\alpha}{1-\mathbb{E} \mathrm{e}^{-\alpha X}}
$$

\subsubsection{Proof of Theorem 4.1(i) for $\alpha=0$}

For $\alpha=0$, relation (4.3) becomes

$$
\lim _{x \rightarrow \infty} \frac{\mathbb{P}(M>x)}{\int_{x}^{\infty} \bar{F}(y) \mathrm{d} y}=\frac{1}{\mu}
$$

To derive the asymptotic upper bound, we still use (4.19). By Proposition 4.6, relation (4.20) holds for arbitrarily small $\varepsilon, \delta>0$ and all large $k$. With $k$ specified in (4.20), by $F_{e} \in \mathcal{L}$ we have

$$
K_{2}(x, k, \delta) \leq \frac{1}{\mu(1-\delta)} \int_{x+(k-1) \mu(1-\delta)}^{\infty} \bar{F}(u) \mathrm{d} u \sim \frac{1}{\mu(1-\delta)} \int_{x}^{\infty} \bar{F}(y) \mathrm{d} y
$$

while by Lemmas 2.24(i) and 2.22,

$$
K_{1}(x, k) \sim k \bar{F}(x)=o(1) \int_{x}^{\infty} \bar{F}(y) \mathrm{d} y
$$

Plugging (4.20), (4.26), and (4.27) into (4.19) and using the arbitrariness of $\varepsilon, \delta>0$, we have

$$
\limsup _{x \rightarrow \infty} \frac{\mathbb{P}(M>x)}{\int_{x}^{\infty} \bar{F}(y) \mathrm{d} y} \leq \frac{1}{\mu}
$$


Next, we consider the asymptotic lower bound. For arbitrarily small $\varepsilon, \delta>0$, by Lemma 4.3 there exists some constant $C>0$ such that inequality (4.7) holds. Write $E_{n}=\left\{n(\mu-\delta)-C \leq S_{n} \leq n(\mu+\delta)+C\right\}$ for $n=0,1, \ldots$ Then by Bonferroni's inequality again,

$$
\begin{aligned}
\mathbb{P}(M>x) \geq & \mathbb{P}\left(\bigcup_{n=1}^{\infty}\left(\left(Y_{n}-S_{n-1}>x\right) \bigcap E_{n-1}\right)\right) \\
\geq & \sum_{n=1}^{\infty} \mathbb{P}\left(\left(Y_{n}-S_{n-1}>x\right) \bigcap E_{n-1}\right) \\
& -\sum_{1 \leq n<m<\infty} \mathbb{P}\left(\left(Y_{n}-S_{n-1}>x\right) \bigcap\left(Y_{m}-S_{m-1}>x\right) \bigcap E_{n-1} \bigcap E_{m-1}\right) \\
\geq & (1-\varepsilon) \sum_{n=1}^{\infty} \bar{F}(x+(n-1)(\mu+\delta)+C) \\
& -\sum_{1 \leq n<m<\infty} \bar{F}(x+(n-1)(\mu-\delta)-C) \bar{F}(x+(m-1)(\mu-\delta)-C) \\
\geq & \frac{1-\varepsilon}{\mu+\delta} \int_{x+C}^{\infty} \bar{F}(y) \mathrm{d} y-\left(\frac{1}{\mu-\delta} \int_{x-(\mu-\delta)-C}^{\infty} \bar{F}(y) \mathrm{d} y\right)^{2} .
\end{aligned}
$$

Since $F_{e} \in \mathcal{L}$, by the arbitrariness of $\varepsilon, \delta>0$ it follows that

$$
\liminf _{x \rightarrow \infty} \frac{\mathbb{P}(M>x)}{\int_{x}^{\infty} \bar{F}(y) \mathrm{d} y} \geq \frac{1}{\mu}
$$

\subsection{Proof of Theorem 4.1(ii)}

\subsubsection{Preliminary Results}

We establish the counterparts of Propositions 4.6 and 4.7 for the case $\alpha>0$, respectively.

Proposition 4.8. Under the conditions of Theorem 4.1(ii) for the case $\alpha>0$, relation (4.11) holds for arbitrarily small $\varepsilon>0,0<\delta<1$ arbitrarily close to 1 , and all large $k$. 
Proof. For $0<\delta, d<1$, introduce the maximum $M_{\delta}=\sup _{n \geq 1} \sum_{i=1}^{n-1}\left(\mu(1-d \delta)-X_{i}\right)$, which is finite almost surely. For every $n \geq k+1$, we derive

$$
S_{n-1}=(n-1) \mu(1-d \delta)-\sum_{i=1}^{n-1}\left(\mu(1-d \delta)-X_{i}\right) \geq(n-1) \mu(1-d \delta)-M_{\delta}
$$

Therefore, for every $k \geq 1$,

$$
\begin{aligned}
& \sum_{n=k+1}^{\infty} \mathbb{P}\left(Y_{n}-S_{n-1}>x, S_{n-1}<(n-1) \mu(1-\delta)\right) \\
\leq & \sum_{n=k+1}^{\infty} \mathbb{P}\left(Y_{n}-(n-1) \mu(1-d \delta)+M_{\delta}>x, M_{\delta}>k \mu(1-d) \delta\right) \\
= & \int_{k \mu(1-d) \delta}^{\infty} \sum_{n=k+1}^{\infty} \bar{F}(x-y+(n-1) \mu(1-d \delta)) \mathbb{P}\left(M_{\delta} \in \mathrm{d} y\right) \\
\leq & \frac{1}{\mu(1-d \delta)}\left(\int_{k \mu(1-d) \delta}^{x}+\int_{x}^{\infty}\right)\left(\int_{x-y}^{\infty} \bar{F}(u) \mathrm{d} u\right) \mathbb{P}\left(M_{\delta} \in \mathrm{d} y\right) \\
\leq & \frac{1}{\mu(1-d \delta)}\left(\nu_{F} \int_{k \mu(1-d) \delta}^{x} \overline{F_{e}}(x-y)+\int_{x}^{\infty}\left(y-x+\nu_{F}\right)\right) \mathbb{P}\left(M_{\delta} \in \mathrm{d} y\right) .
\end{aligned}
$$

To apply Lemma 4.5 , we need to choose $\delta$ and $d$ close to 1 such that $\mathbb{E}^{\alpha(\mu(1-d \delta)-X)}<1$. Let $F^{*}$ be a distribution defined as $F^{*}(x)=F(x-\mu(1-d \delta))$. Then, $\mathbb{P}(\mu(1-d \delta)-$ $X>x)=o\left(\overline{F^{*}}(x)\right)$ and $F_{e}^{*} \in \mathcal{S}(\alpha)$. By Lemma 4.5, we have

$$
\mathbb{P}\left(M_{\delta}>x\right)=o\left(\overline{F_{e}^{*}}(x)\right)=o\left(\overline{F_{e}}(x)\right)
$$

By Lemma 2.24(ii) and the local uniformity of the convergence in relation (2.14), it holds for arbitrarily fixed $k \geq 1$ that

$$
\begin{aligned}
\int_{k \mu(1-d) \delta}^{x} \overline{F_{e}}(x-y) \mathbb{P}\left(M_{\delta} \in \mathrm{d} y\right) & \leq\left(\int_{0-}^{\infty}-\int_{0-}^{k \mu(1-d) \delta}\right) \overline{F_{e}}(x-y) \mathbb{P}\left(M_{\delta} \in \mathrm{d} y\right) \\
& \sim \overline{F_{e}}(x) \mathbb{E}^{\alpha M_{\delta}} 1_{\left\{M_{\delta}>k \mu(1-d) \delta\right\}}
\end{aligned}
$$


Moreover, by Lemma 2.22,

$$
\int_{x}^{\infty}(y-x) \mathbb{P}\left(M_{\delta} \in \mathrm{d} y\right)=\int_{x}^{\infty} \mathbb{P}\left(M_{\delta}>y\right) \mathrm{d} y=o(1) \int_{x}^{\infty} \overline{F_{e}}(y) \mathrm{d} y=o\left(\overline{F_{e}}(x)\right) .
$$

Plugging (4.29)-(4.31) into (4.28) yields the desired assertion.

Proposition 4.9. Under the conditions of Theorem 4.1(ii) for the case $\alpha>0$, relation (4.16) holds for each $k=2,3, \ldots$

Proof. By Lemma 2.22, $F_{e} \in \mathcal{S}(\alpha)$ for some $\alpha>0$ implies $F \in \mathcal{S}(\alpha)$. When $1=n<$ $m \leq k$, for arbitrarily fixed $D>0$, we have

$$
\begin{aligned}
& \mathbb{P}\left(Y_{1}>x, Y_{m}-S_{m-1}>x\right) \\
\leq & \mathbb{P}\left(-S_{m-1}>x-D\right)+\mathbb{P}\left(Y_{1}>x, Y_{m}-S_{m-1}>x,-S_{m-1} \leq x-D\right) \\
\leq & \mathbb{P}\left(-S_{m-1}>x-D\right)+\mathbb{P}\left(Y_{1}>x, Y_{m}>D\right) .
\end{aligned}
$$

By Lemma 2.24(ii),

$$
\mathbb{P}\left(-S_{m-1}>x-D\right)=o(\bar{F}(x-D))=o(\bar{F}(x))
$$

Substitute this into (4.32) then notice that $D$ can be arbitrarily large. It follows that

$$
\mathbb{P}\left(Y_{1}>x, Y_{m}-S_{m-1}>x\right)=o(\bar{F}(x))=o\left(\overline{F_{e}}(x)\right)
$$

Similarly, when $1<n<m \leq k$, for arbitrarily fixed $D>0$,

$$
\begin{aligned}
& \mathbb{P}\left(Y_{n}-S_{n-1}>x, Y_{m}-S_{m-1}>x\right) \\
\leq & \mathbb{P}\left(-S_{n-1}>x-D\right)+\int_{-\infty}^{x-D} \mathbb{P}\left(Y_{n}>x-y, Y_{m}-S_{n, m-1}>x-y\right) \mathbb{P}\left(-S_{n-1} \in \mathrm{d} y\right),
\end{aligned}
$$


where $S_{n, m-1}=\sum_{i=n}^{m-1} X_{i}$ as before. By (4.33), for arbitrarily small $\varepsilon>0$, choose $D>0$ such that

$$
\mathbb{P}\left(Y_{n}>x, Y_{m}-S_{n, m-1}>x\right) \leq \varepsilon \overline{F_{e}}(x)
$$

for $1<n<m \leq k$ and all $x \geq D$. Using this inequality and Lemma 2.24(ii), we obtain that

$$
\begin{aligned}
\mathbb{P}\left(Y_{n}-S_{n-1}>x, Y_{m}-S_{m-1}>x\right) & \leq o\left(\overline{F_{e}}(x)\right)+\varepsilon \int_{-\infty}^{x-D} \overline{F_{e}}(x-y) \mathbb{P}\left(-S_{n-1} \in \mathrm{d} y\right) \\
& \lesssim \varepsilon \mathbb{E}^{-\alpha S_{n-1}} \overline{F_{e}}(x)
\end{aligned}
$$

This proves that

$$
\mathbb{P}\left(Y_{n}-S_{n-1}>x, Y_{m}-S_{m-1}>x\right)=o\left(\overline{F_{e}}(x)\right)
$$

A combination of (4.33) and (4.34) gives (4.16).

\subsubsection{Proof of Theorem 4.1(ii)}

The proof for the case $\alpha>0$ can be given by copying the proof of Theorem 4.1(i) for the case $\alpha>0$ with only modifications that we use Lemma 2.24(ii) and Propositions 4.8 and 4.9 instead of Lemma 2.24(i) and Propositions 4.6 and 4.7.

Consider the case $\alpha=0$ and we aim at relation (4.25). The proof of the asymptotic lower bound is the same as that in Theorem 4.1(i). The proof of the asymptotic upper bound can be found in Palmowski and Zwart (2007). Nevertheless, for the sake of self-containedness, we copy their proof here.

For some arbitrarily large but fixed $\zeta>0$, define

$$
Z=(-X) 1_{\{(-X) \vee Y \leq \zeta\}}+((-X) \vee Y) 1_{\{(-X) \vee Y>\zeta\}}
$$


Clearly, $Z=Z(\zeta)$ converges to $-X$ in distribution as $\zeta \rightarrow \infty$ and $\mathbb{E} Z<0$ for all large $\zeta$. Moreover, it is easy to see that the relation $\mathbb{P}(Z>x) \sim \bar{F}(x)$ holds for arbitrarily fixed $\zeta$. Define $Z_{n}$ in a similar way in terms of $X_{n}$ and $Y_{n}, n=1,2, \ldots$, so that $\left\{Z_{n}, n=1,2, \ldots\right\}$ forms a sequence of i.i.d. copies of $Z$. Then, we arrive at a key inequality of Palmowski and Zwart (2007) that

$$
M=\sup _{n \geq 1}\left(Y_{n}-S_{n-1}\right) \leq \sup _{n \geq 1} \sum_{i=1}^{n-1} Z_{i}+\zeta .
$$

Therefore, by Lemma 4.4(ii),

$$
\mathbb{P}(M>x) \leq \mathbb{P}\left(\sup _{n \geq 1} \sum_{i=1}^{n-1} Z_{i}>x-\zeta\right) \sim-\frac{1}{\mathbb{E} Z} \int_{x-\zeta}^{\infty} \bar{F}(y) \mathrm{d} y .
$$

Since $F_{e} \in \mathcal{S}$ and $\zeta$ can be arbitrarily large, it follows that

$$
\mathbb{P}(M>x) \lesssim \frac{1}{\mu} \int_{x}^{\infty} \bar{F}(y) \mathrm{d} y
$$

\subsection{Simulations}

In this section, we present two simulation results for Theorem 4.1, one for light-tailed $F$ and the other for heavy-tailed $F$. We want to see from the two special cases how fast the convergence in relation (4.3) is.

\section{(i) Light-tailed Case:}

Let $(X, Y)$ have the following marginal density functions

$$
f_{X}(x)=\mathrm{e}^{4 x} 1_{\{x<0\}}+\frac{3}{2} \mathrm{e}^{-2 x} 1_{\{x \geq 0\}}, \quad f_{Y}(y)=\mathrm{e}^{-x} 1_{\{x \geq 0\}} .
$$

Then, $F_{Y} \in \mathcal{L}(1), \mathbb{E} X=5 / 16>0, \mathbb{E} X^{2}<\infty$, and $\mathbb{E} \mathrm{e}^{-\beta X}<1$ for all $\beta \in(1,5 / 2)$. Hence, all conditions of Theorem 4.1(i) are satisfied. To model the dependence structure between $X$ and $Y$, we apply the so-called Farlie-Gumbel-Morgenstern copula, 
i.e., $C\left(u_{1}, u_{2}\right)=u_{1} u_{2}\left(1+c\left(1-u_{1}\right)\left(1-u_{2}\right)\right)$ with $c \in[-1,1]$ and $u_{1}, u_{2} \in[0,1]$. In other words,

$$
F_{X, Y}(x, y)=F_{X}(x) F_{Y}(y)\left(1+c \overline{F_{X}}(x) \overline{F_{Y}}(y)\right)
$$

where $F_{X, Y}(\cdot), F_{X}(\cdot)$, and $F_{Y}(\cdot)$ are the corresponding joint and marginal distributions. We use the following algorithm (see Johnson (1986)) to generate random values for $F_{X}(\cdot)$ and $F_{Y}(\cdot)$ :

1. Generate two independent uniform $(0,1)$ variates $v_{1}, v_{2}$;

2. Set $a=1+c\left(1-2 v_{1}\right), b=\sqrt{a^{2}-4(a-1) v_{2}}$;

3. Set $u_{1}=v_{1}, u_{2}=2 v_{2} /(a+b)$;

4. Then $\left(u_{1}, u_{2}\right)$ is one outcome of $\left(F_{X}(\cdot), F_{Y}(\cdot)\right)$.

We set $c=1 / 2$ and execute our simulation in $\mathrm{R}$ software. The simulation result is shown in Figure 4.1 below. 


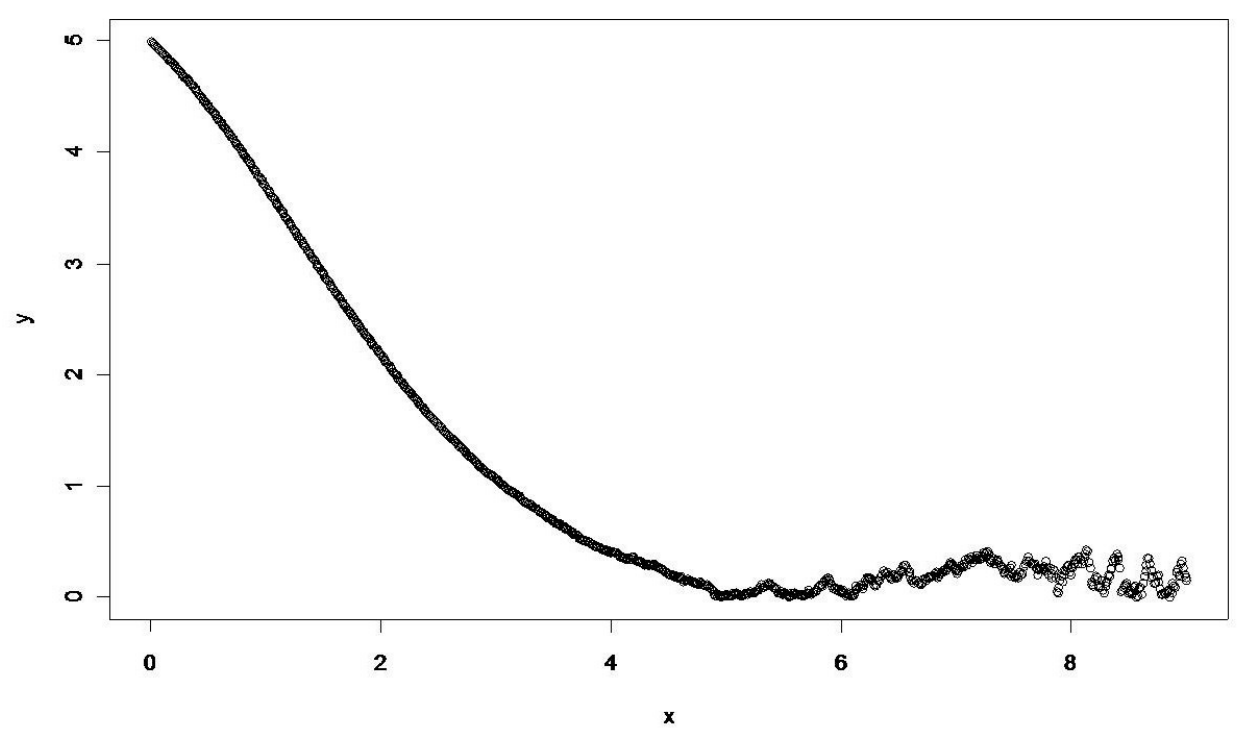

Figure 4.1: $y$-axis represents for each fixed $x$ the LHS substracts the RHS in (4.3) when $Y$ is $\operatorname{Exp}(1)$ distributed.

\section{(ii) Heavy-tailed Case:}

Let $(X, Y)$ have the following marginal distributions

$$
F_{X}(x)=\frac{1}{2} \mathrm{e}^{x} 1_{\{x<0\}}+\left(1-\frac{1}{2} \mathrm{e}^{-x / 2}\right) 1_{\{x \geq 0\}}, \quad F_{Y}(y)=\left(1-\mathrm{e}^{-\sqrt{y}}\right) 1_{\{x \geq 0\}} .
$$

Here $Y$ follows a heavy-tailed Weibull distribution with shape parameter $\tau=1 / 2$. It is easy to see that the equilibrium distribution of $F_{Y}$ is subexponential (see Example 1.4.7 of Embrechts et al. (1997)), $\mathbb{E} X=1 / 2>0$, and $\mathbb{P}(-X>x)=o\left(\overline{F_{Y}}(x)\right)$. Hence, all conditions of Theorem 4.1(ii) are satisfied. We use the same Farlie-GumbelMorgenstern copula as above to model the dependence structure between $X$ and $Y$. The simulation result is shown in Figure 4.2 below. 


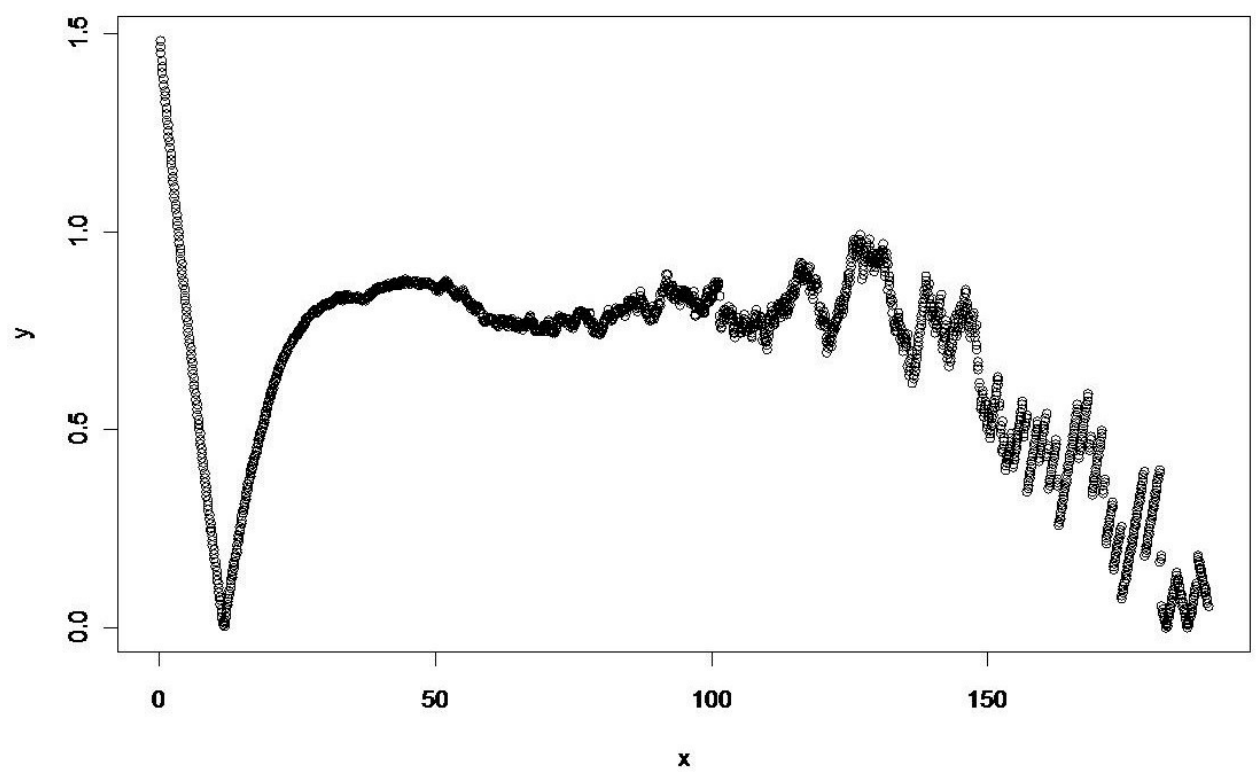

Figure 4.2: $y$-axis represents for each fixed $x$ the LHS substracts the RHS in (4.3) when $Y$ is heavy-tailed Weibull distributed. 


\section{CHAPTER 5 THE LÉVY INSURANCE RISK MODEL UNDER TAXATION}

In this chapter, we use a general Lévy process to model the underlying surplus process of an insurance company in a world without economic factors. This so-called Lévy risk model has recently attracted a lot of attention in the insurance literature. We are particularly interested in how to capture the impact of tax payments on the ruin probability. In a series of recent papers by Albrecher and his coauthors, it is assumed that taxes are paid at a certain fixed rate immediately when the surplus of the company is at a running maximum. In reality, however, taxes are usually paid periodically (e.g. monthly, semi-annually, or annually). Therefore, we introduce periodic taxation under which the company pays tax at a fixed rate on its net income during each period. As main results, we derive for the ruin probability several explicit asymptotic relations, in which the prefactor varies with the tax rate, reflecting the impact of tax payments. This chapter is based on the joint research paper Hao and Tang (2009).

\section{$5.1 \quad$ Introduction}

As mentioned in Chapter 4, the ruin probability of an insurance company is the probability that its surplus process falls below 0 at some time. Let $U=\left(U_{t}\right)_{t \geq 0}$ be a stochastic process, with $U_{0}=x>0$, representing the underlying surplus process in a world without economic factors (tax, reinsurance, investment, etc.) of an insurance

company. Assuming that $U$ is a compound Poisson process with positive drift and 
that taxes are paid at a fixed rate $\gamma \in[0,1)$ whenever $U$ is at a running maximum (called the loss-carry-forward taxation), Albrecher and Hipp (2007) and Albrecher et al. (2009) proved the following strikingly simple relationship between $\psi_{\gamma}(x)$ and $\psi_{0}(x)$, the ruin probabilities with and without tax:

$$
\psi_{\gamma}(x)=1-\left(1-\psi_{0}(x)\right)^{1 /(1-\gamma)}
$$

Albrecher et al. (2008b) further showed that the tax identity (5.1) still holds for a spectrally negative Lévy surplus process $U$ under the loss-carry-forward taxation. Also, Albrecher et al. (2008a) proved a similar tax identity for a dual surplus process $U$ with general inter-innovation times and exponential innovation sizes under the same type of taxation.

All these papers cited above assume the loss-carry-forward taxation. In reality, however, taxes are usually paid periodically (e.g. monthly, semi-annually, or annually). Furthermore, if the surplus process contains a diffusion part, then the moments of running maxima do not form any continuous time interval. In this case, the losscarry-forward type taxation is rather unrealistic, as was also commented by Albrecher and Hipp (2007). Figure 5.1 below shows how the loss-carry-forward taxation affects the underlying surplus process in the compound Poisson model.

In this chapter, we introduce periodic taxation as well as reinsurance to the risk model. Precisely, we assume that at each discrete moment $n=1,2, \ldots$, the company, given that it survives at that moment, pays tax at rate $\gamma \in[0,1)$ on its net income during the period $(n-1, n]$ and it gets paid by reinsurance at rate $\delta \in[0,1)$ on its net loss during the period $(n-1, n]$. We are interested in the influence of such 


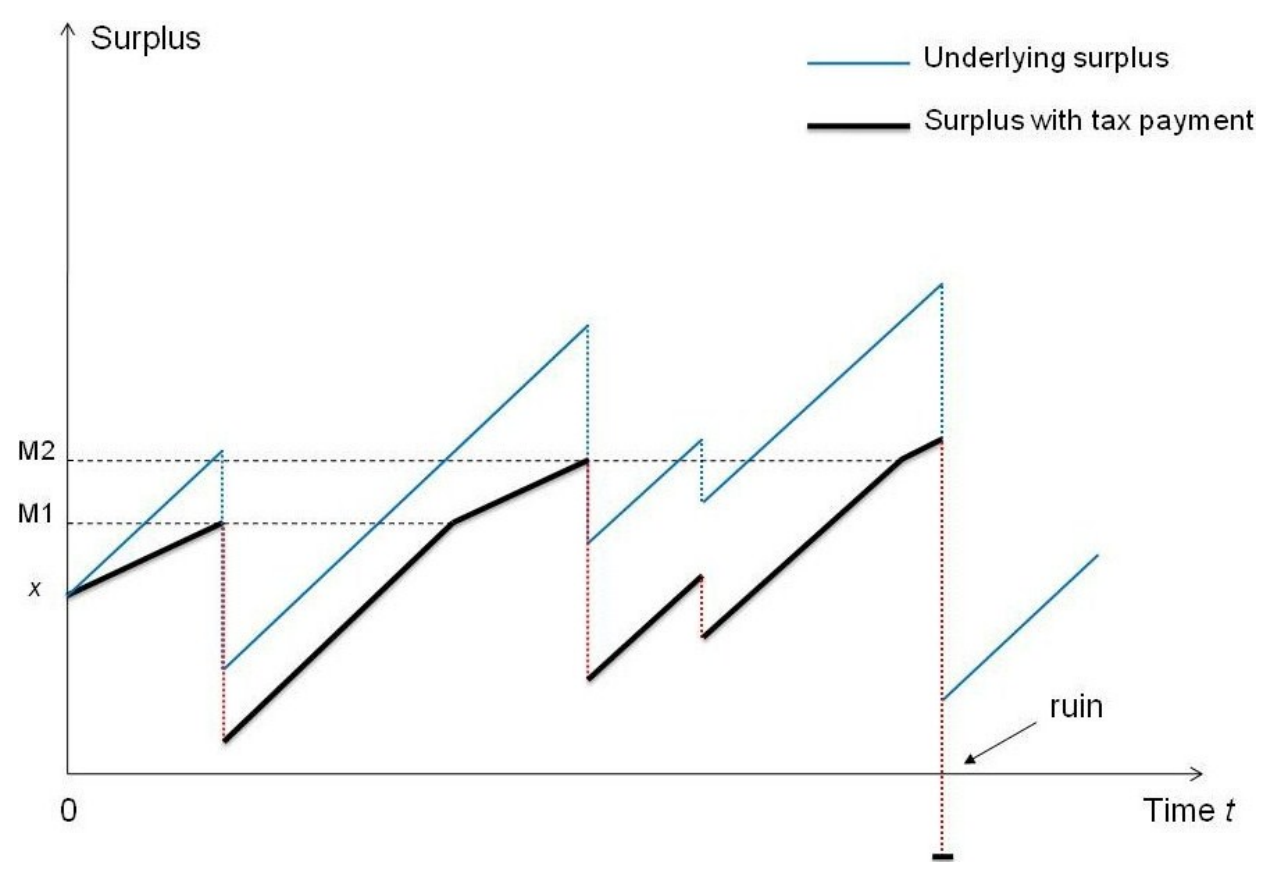

Figure 5.1: Loss-carry-forward taxation

a scheme of taxation rule and reinsurance policy on the asymptotic behavior of the ruin probability. Figure 5.2 below shows how the periodic taxation affects the same underlying surplus process as that in Figure 5.1.

Let us briefly compare these two types of taxation. Under the loss-carryforward taxation, as long as the surplus does not hit its historical peak, the insurance company can legally evade any tax payment possibly for a long time, even if it makes profits every single period during that time. While under the periodic taxation, the insurance company has to pay tax whenever it survives and its net income is positive in that period. Hence, the latter imposes a more strict taxation rule and produces more significant impact on the ruin probability than the former does. This will be demonstrated in Section 5.2. 


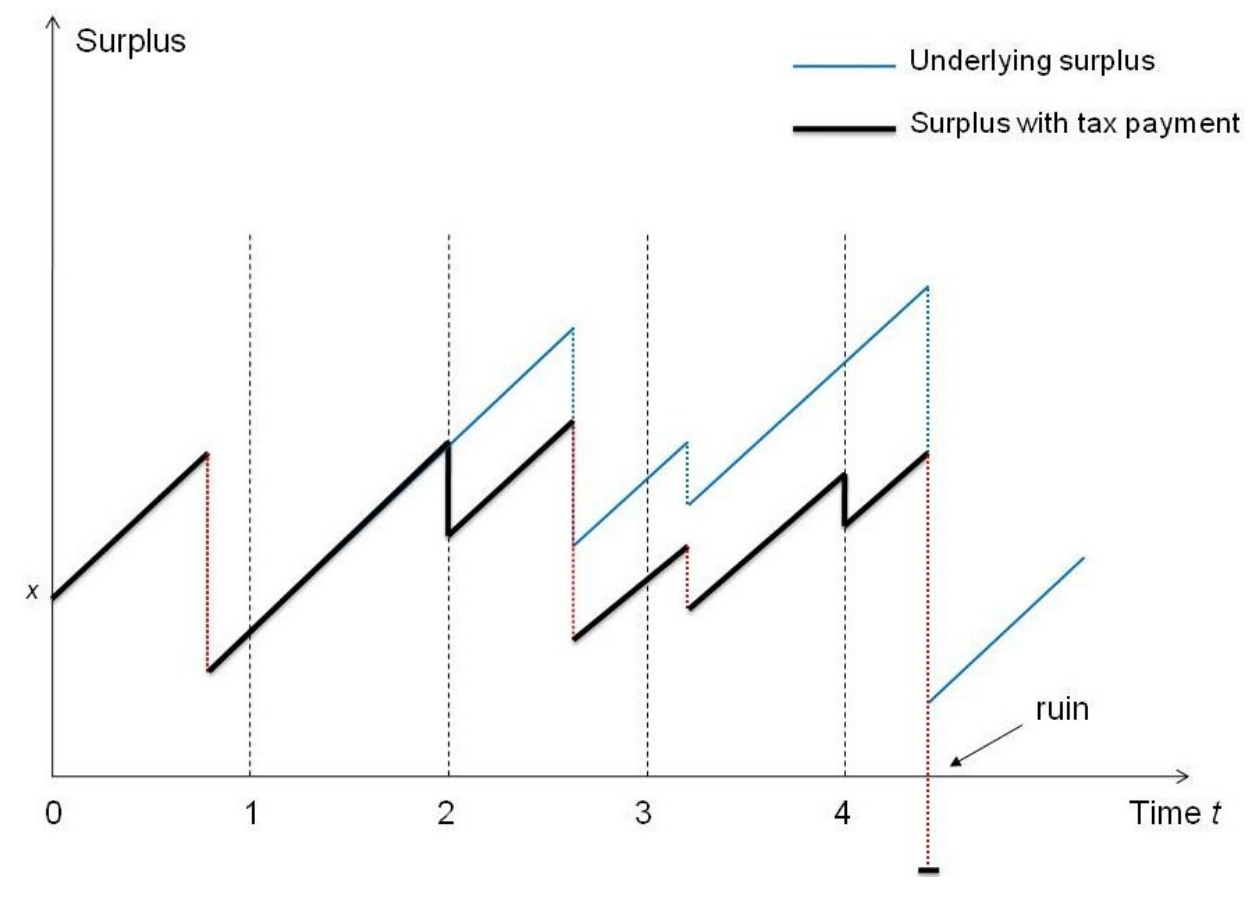

Figure 5.2: Periodic taxation

It is convenient for us to look at the loss process before tax and reinsurance,

$$
L_{t}=x-U_{t}, \quad t \geq 0 .
$$

For each $n=1,2, \ldots$, the maximal net loss and the net loss within the period $(n-1, n]$ are, respectively,

$$
Y_{n}=\sup _{n-1 \leq t \leq n}\left(L_{t}-L_{n-1}\right), \quad Z_{n}=L_{n}-L_{n-1} .
$$

After introducing the periodic taxation at rate $0 \leq \gamma<1$ and reinsurance at rate $0 \leq \delta<1$, the loss of the company within the period $(n-1, n]$ becomes

$$
X_{n}=Z_{n}+\gamma Z_{n}^{-}-\delta Z_{n}^{+}=(1-\delta) Z_{n}^{+}-(1-\gamma) Z_{n}^{-} .
$$


Then, it is easy to see that the ruin probability in this situation is equal to

$$
\psi_{\gamma, \delta}(x)=\mathbb{P}\left(\sup _{n \geq 1}\left(\sum_{k=1}^{n-1} X_{k}+Y_{n}\right)>x\right) .
$$

Notice that we have used $\psi_{\gamma}(x)$ (with only one subscript) for the ruin probability under the loss-carry-forward taxation and used $\psi_{\gamma, \delta}(x)$ (with two subscripts) for the ruin probability under the periodic taxation and reinsurance. We shall let the notation speak for itself.

In this chapter, we shall assume that the loss process $L$ is a Lévy process with mean $\mathbb{E} L_{1}=-\mu<0$ (so that it converges to $-\infty$ almost surely). Consequently, the random pairs $\left(X_{n}, Y_{n}\right), n=1,2, \ldots$, appearing in (5.2) are i.i.d. copies of the random pair

$$
(X, Y) \stackrel{d}{=}\left((1-\delta) L_{1}^{+}-(1-\gamma) L_{1}^{-}, \sup _{0 \leq t \leq 1} L_{t}\right)
$$

Write $\mu_{+}=\mathbb{E} L_{1}^{+}$and $\mu_{-}=\mathbb{E} L_{1}^{-}$, which are assumed to be finite. Throughout this chapter, we always choose $\gamma \in[0,1)$ and $\delta \in[0,1)$ such that

$$
\mathbb{E} X=(1-\delta) \mu_{+}-(1-\gamma) \mu_{-}<0
$$

so that the insurance company still has positive expected profits under such a scheme of taxation rule and reinsurance policy and that the ruin is not certain.

For a Lévy measure $\rho$, write $\bar{\rho}(x)=\rho((x, \infty))$ for $x \geq 0$. When $\bar{\rho}(1)>0$, introduce $\Pi(\cdot)=(\bar{\rho}(1))^{-1} \rho(\cdot) 1_{(1, \infty)}$, which is a proper probability measure on $(1, \infty)$. We shall assume that the Lévy measure $\rho$ has a tail $\bar{\rho}$ asymptotic to a subexponential tail, a convolution-equivalent tail, and an exponential-like tail. These are natural assumptions when studying the tail probability of the Lévy process. In risk theory, 
these assumptions have recently been used by e.g. Klüppelberg et al. (2004) and Doney and Kyprianou (2006).

In the rest of this chapter we present our main results in Sections 5.2-5.4 for the cases that the Lévy measure $\rho$ of the loss process $L$ has a subexponential tail, a convolution-equivalent tail, and an exponential-like tail, respectively.

\subsection{The Case of Subexponential Tails}

In our first main result below we look at the case that the Lévy measure $\rho$ has a subexponential tail.

Theorem 5.1. Consider the Lévy insurance model introduced in Section 5.1. If both $\Pi$ and $\Pi_{e}$ belong to the class $\mathcal{S}$ (which are satisfied when $\Pi \in \mathcal{S}^{*}$ ), then for every $0 \leq \gamma<1$ and $0 \leq \delta<1$ for which relation (5.4) holds, we have

$$
\psi_{\gamma, \delta}(x) \sim \frac{1}{(1-\gamma) \mu_{-}-(1-\delta) \mu_{+}} \int_{x}^{\infty} \bar{\rho}(y) \mathrm{d} y .
$$

Klüppelberg et al. (2004) systematically studied the asymptotic behavior of the ruin probability in the Lévy insurance model without tax or reinsurance. Restricting to the case that $L$ is spectrally positive with Lévy measure $\rho$ such that $\Pi \in \mathcal{S}^{*}$, we see that Theorem 6.2(i) of Klüppelberg et al. (2004) corresponds to our Theorem 5.1 with $\gamma=\delta=0$.

Clearly, the tax identity (5.1) under the loss-carry-forward taxation implies that

$$
\psi_{\gamma}(x) \sim \frac{1}{1-\gamma} \psi_{0}(x)
$$


see also Albrecher and Hipp (2007). While under our periodic taxation, substituting $\delta=0$ to $(5.5)$ yields that

$$
\psi_{\gamma, 0}(x) \sim \frac{1}{1-\gamma \frac{\mu_{-}}{\mu_{-}-\mu_{+}}} \psi_{0,0}(x)
$$

Note that $\psi_{0}(x)$ in (5.6) and $\psi_{0,0}(x)$ in (5.7) are identical. The coefficients in relations (5.6) and (5.7) respectively capture the impact of the two taxation rules on the asymptotic behavior of the ruin probability. Now that $\mu_{-} /\left(\mu_{-}-\mu_{+}\right)>1$ in (5.7), comparing (5.6) with (5.7) we conclude that periodic taxation produces more significant impact on the ruin probability than the loss-carry-forward taxation does.

To prove Theorem 5.1, we need the following two lemmas:

Lemma 5.2. Let $L$ be a Lévy process with Lévy measure $\rho$ such that $\Pi \in \mathcal{S}$. Then,

$$
\mathbb{P}\left(\sup _{0 \leq t \leq 1} L_{t}>x\right) \sim \bar{\rho}(x)
$$

Lemma 5.3 (Theorem 1 of Palmowski and Zwart (2007)). Let random pairs $\left(X_{n}, Y_{n}\right)$, $n=1,2, \ldots$, be i.i.d. copies of a random pair $(X, Y)$. Denote $M=X \vee Y$. If $-\infty<\mathbb{E} X<0, \mathbb{E} M<\infty$, and $\int_{x}^{\infty} \mathbb{P}(M>y) \mathrm{d} y$ is asymptotic to a subexponential tail, then,

$$
\mathbb{P}\left(\sup _{n \geq 1}\left(\sum_{k=1}^{n-1} X_{k}+Y_{n}\right)>x\right) \sim \frac{1}{|\mathbb{E} X|} \int_{x}^{\infty} \mathbb{P}(M>y) \mathrm{d} y
$$

Lemma 5.2 is an implication of Theorem 3.1 of Rosiński and Samorodnitsky (1993) (see the example of Lévy motion on their page 1006).

Proof of Theorem 5.1. Recall (5.2), where the random pairs $\left(X_{n}, Y_{n}\right), n=1,2, \ldots$, are i.i.d. copies of the random pair $(X, Y)$ given in (5.3). Use the notation $M=X \vee Y$ 
in Lemma 5.3. Since $\Pi \in \mathcal{S}$, from Lemma 5.2 we have

$$
\mathbb{P}(Y>x) \sim \bar{\rho}(x)
$$

It is clear that $Y \geq L_{1}^{+} \geq X^{+}$. Hence by $(5.9)$ and $\Pi_{I} \in \mathcal{S}$,

$$
\int_{x}^{\infty} \mathbb{P}(M>y) \mathrm{d} y=\int_{x}^{\infty} \mathbb{P}(Y>y) \mathrm{d} y \sim \int_{x}^{\infty} \bar{\rho}(y) \mathrm{d} y
$$

a subexponential tail. Then by Lemma 5.3, we obtain (5.5).

\subsection{The Case of Convolution-equivalent Tails}

Next, we consider the case that the Lévy measure $\rho$ has a light tail such that $\Pi \in \mathcal{S}(\alpha)$ for some $\alpha>0$.

Theorem 5.4. Consider the Lévy insurance model introduced in Section 5.1. Assume $\mathbb{E} L_{1}^{2}<\infty$ and $\Pi \in \mathcal{S}(\alpha)$ for some $\alpha>0$. If $0 \leq \gamma<1$ and $0<\delta<1$ are such that

$$
\mathbb{E e}^{\alpha^{\prime}\left((1-\delta) L_{1}^{+}-(1-\gamma) L_{1}^{-}\right)}<1
$$

for some $\alpha^{\prime}>\alpha$, then,

$$
\psi_{\gamma, \delta}(x) \sim \frac{c_{\alpha}}{1-\mathbb{E} \mathrm{e}^{\alpha\left((1-\delta) L_{1}^{+}-(1-\gamma) L_{1}^{-}\right)}} \bar{\rho}(x)
$$

where the constant $c_{\alpha}$ is defined as

$$
c_{\alpha}=\lim _{x \rightarrow \infty} \frac{\mathbb{P}\left(\sup _{0 \leq t \leq 1} L_{t}>x\right)}{\bar{\rho}(x)} \in(0, \infty) .
$$

The existence of the limit $c_{\alpha}$ in (5.12) was proved by Braverman and Samorodnitsky (1995); see the following lemma. Condition (5.10) is feasible because $\mathbb{E} \mathrm{e}^{\alpha L_{1}^{+}}<$ $\infty$ and $0<\delta<1$ 
Lemma 5.5 (Theorem 3.1 of Braverman and Samorodnitsky (1995)). Let L be a Lévy process with Lévy measure $\rho$ such that $\Pi \in \mathcal{S}(\alpha)$ for some $\alpha>0$. Then,

$$
\lim _{x \rightarrow \infty} \frac{\mathbb{P}\left(\sup _{0 \leq t \leq 1} L_{t}>x\right)}{\bar{\rho}(x)}=c \quad \text { for some } c \in(0, \infty)
$$

By Lemma 2.21, relation (5.11) may be rewritten as

$$
\psi_{\gamma, \delta}(x) \sim \frac{\alpha c_{\alpha}}{1-\mathbb{E} \mathrm{e}^{\alpha\left((1-\delta) L_{1}^{+}-(1-\gamma) L_{1}^{-}\right)}} \int_{x}^{\infty} \bar{\rho}(y) \mathrm{d} y
$$

With the understanding that $c_{0}=1$ by relation (5.8) and that the coefficient in the right-hand side of relation (5.13) converges to $\left((1-\gamma) \mu_{-}-(1-\delta) \mu_{+}\right)^{-1}$ as $\alpha \rightarrow 0$, relation (5.5) in Theorem 5.1 indicates that relation (5.13) still holds when $\alpha=0$.

Recalling the light-tailed case of Theorem 4.1(i) in Chapter 4, we give the proof of Theorem 5.4:

Proof of Theorem 5.4. Use the notation in (5.3). By relation (5.12) and closure of the class $\mathcal{S}(\alpha)$ under tail equivalence, the distribution of $Y$ also belongs to the class $\mathcal{S}(\alpha)$. The moment conditions on $X$ required in Theorem 4.1(i) are clearly satisfied. Then, using Theorem 4.1(i) we immediately obtain (5.11).

To apply Theorem 5.4, a direct problem is how to determine the constant $c_{\alpha}$ in (5.12). This has been a very difficult problem for a Lévy process $L$ whose Lévy measure $\rho$ has a convolution-equivalent tail. For related discussions see Albin and Sundén (2009) and references therein. The following lemma gives an expression for $c_{\alpha}$ : 
Lemma 5.6. Let $L$ be a Lévy process with Lévy measure $\rho$ such that $\Pi \in \mathcal{S}(\alpha)$ for some $\alpha>0$. Then for all $t>0$,

$$
\lim _{x \rightarrow \infty} \frac{\mathbb{P}\left(L_{t}>x\right)}{\bar{\rho}(x)}=t \mathbb{E} \mathrm{e}^{\alpha L_{t}}:=h(t)
$$

There is a unique probability distribution $G$ on $[0,1]$ satisfying $\int_{0}^{1} t^{-1} G(\mathrm{~d} t)<\infty$ with moments given by

$$
\mu_{n}(G)=\frac{v_{n}(n+1) !}{\int_{0}^{1} h(t) \mathrm{d} t}, \quad n=1,2, \ldots
$$

where

$$
v_{n}=\int_{0<t_{1} \leq \cdots \leq t_{n+1} \leq 1} t_{1} \mathbb{E}^{\alpha \min _{1 \leq k \leq n+1} L_{t}} \mathrm{~d} t_{1} \cdots \mathrm{d} t_{n+1} .
$$

Finally,

$$
\lim _{x \rightarrow \infty} \frac{\mathbb{P}\left(\sup _{0 \leq t \leq 1} L_{t}>x\right)}{\bar{\rho}(x)}=\int_{0}^{1} t^{-1} G(\mathrm{~d} t) \int_{0}^{1} h(t) \mathrm{d} t:=c_{\alpha} .
$$

Lemma 5.6 is a combination of Proposition 1.3 and Theorem 2.1 of Braverman (1997). Here we need to point out that the constants $v_{n}$ defined by Braverman (1997) are not correct. This is due to a calculation error in his Lemma 3.1. Indeed, under his assumptions and notation, instead of his relation (3.1) we should have

$$
\mathbb{P}\left(\Sigma_{k}>x, 1 \leq k \leq n\right) \sim \overline{F_{1}}(x) \mathbb{E} \mathrm{e}^{\alpha\left(\min _{1 \leq k \leq n} \Sigma_{k}-X_{1}\right)},
$$

where $\Sigma_{k}=\sum_{i=1}^{k} X_{i}, 1 \leq k \leq n$. Therefore, to qualify his Theorem 2.1, the constants $v_{n}$ should be given by our (5.14) above. However, we remark that the expression for $c_{\alpha}$ given in (5.15) is far from being explicit and can not be evaluated unless $L$ is a subordinator.

To pursue a more explicit expression for $c_{\alpha}$, we then restrict the Lévy process $L$ to a compound Poisson process with negative drift. 
Corollary 5.7. Consider the Lévy insurance model introduced in Section 5.1. Assume

$$
L_{t}=V_{t}-p t, \quad t \geq 0
$$

where $p>0$ represents the constant premium rate and $V=\left(V_{t}\right)_{t \geq 0}$ is a compound Poisson process as given in Section 2.1, i.e., $V_{t}=\sum_{k=1}^{N_{t}} \xi_{k}$, where $N=\left(N_{t}\right)_{t \geq 0}$ is a Poisson process with intensity $\lambda>0$ and $\xi_{1}, \xi_{2}, \ldots$ are i.i.d. random variables independent of $N$ and with common distribution $F$ on $(-\infty, \infty)$. Suppose that $F$ has a bounded density $f \in \mathcal{S} d(\alpha)$ for some $\alpha>0$ and that condition (5.10) holds. Then,

$$
\psi_{\gamma, \delta}(x) \sim \frac{\lambda c_{\alpha}}{1-\mathbb{E} \mathrm{e}^{\alpha\left((1-\delta) L_{1}^{+}-(1-\gamma) L_{1}^{-}\right)}} \bar{F}(x)
$$

with the constant $c_{\alpha}$ given by

$$
c_{\alpha}=\mathrm{e}^{\lambda\left(\mathbb{E} \mathrm{e}^{\alpha \xi}-1\right)-\alpha p}+\alpha \int_{0}^{1}\left(\int_{0}^{t} \mathbb{P}\left(\sum_{k=1}^{N_{t}} \xi_{k} \leq p s\right) \mathrm{d} s\right) \frac{1-t}{t} \mathrm{e}^{\lambda(1-t)\left(\mathbb{E} \mathrm{e}^{\alpha \xi}-1\right)-\alpha p(1-t)} \mathrm{d} t .
$$

For example, if $F=I G(\mu, \nu)$ with density given by (2.18), i.e.,

$$
f(x)=\left(\frac{\nu}{2 \pi x^{3}}\right)^{1 / 2} \exp \left\{\frac{-\nu(x-\mu)^{2}}{2 \mu^{2} x}\right\}, \quad \mu, \nu, x>0,
$$

then we can appropriately choose the constants $p, \gamma$, and $\delta$ such that condition $(5.10)$ is satisfied.

While the expression for $c_{\alpha}$ defined in (5.17) is still not completely explicit, with the only unknown part $\int_{0}^{t} \mathbb{P}\left(\sum_{k=1}^{N_{t}} \xi_{k} \leq p s\right) \mathrm{d} s$ for $0<t \leq 1$, it is simple enough for simulations, especially when $\xi$ follows an inverse Gaussian distribution.

Next we prove Corollary 5.7. Let $F(\cdot, t)$ be the distribution of aggregate claims,

$$
F(x, t)=\mathbb{P}\left(\sum_{k=1}^{N_{t}} \xi_{k} \leq x\right)
$$


and let $f(\cdot, t)$ be its density. Write $Y_{t}=\sup _{0 \leq s \leq t} L_{s}$. Then $Y_{1}=Y$. The lemma below is a restatement of Theorems 2.1 and 2.2 of Asmussen (2000):

Lemma 5.8. For the compound Poisson model (5.16), we have

$$
\mathbb{P}\left(Y_{t} \leq 0\right)=\frac{1}{t} \int_{0}^{t} F(p s, t) \mathrm{d} s, \quad t>0
$$

and

$$
1-\mathbb{P}\left(Y_{T}>x\right)=F(x+p T, T)-\int_{0}^{T} \mathbb{P}\left(Y_{T-t} \leq 0\right) f(x+p t, t) \mathrm{d} t, \quad T>0
$$

Proof of Corollary 5.7. By Theorem 5.4, it suffices to verify (5.17). By Lemma 5.8 , we have

$$
\mathbb{P}(Y>x)=\bar{F}(x+p, 1)+\int_{0}^{1} \mathbb{P}\left(Y_{1-t} \leq 0\right) f(x+p t, t) \mathrm{d} t
$$

Since $f \in \mathcal{S} d(\alpha)$ for $\alpha>0$ implies $F \in \mathcal{S}(\alpha)$, we apply the dominated convergence theorem justified by Lemma 2.25 to obtain that

$$
\begin{aligned}
\bar{F}(x+p, 1) & =\sum_{n=1}^{\infty} \mathbb{P}\left(\sum_{k=1}^{n} \xi_{k}>x+p\right) \mathbb{P}\left(N_{1}=n\right) \\
& \sim \lambda \mathrm{e}^{\lambda\left(\mathbb{E} \mathrm{e}^{\alpha \xi}-1\right)} \bar{F}(x+p) \sim \lambda \mathrm{e}^{\lambda\left(\mathbb{E}^{\alpha \xi}-1\right)-\alpha p} \bar{F}(x) .
\end{aligned}
$$

Similarly, by Lemma 2.26, for each fixed $t \in(0,1]$,

$$
f(x+p t, t) \sim \sum_{n=1}^{\infty} \frac{(\lambda t)^{n}}{n !} \mathrm{e}^{-\lambda t} \cdot n\left(\mathbb{E} \mathrm{e}^{\alpha \xi}\right)^{n-1} f(x+p t) \sim \lambda t \mathrm{e}^{\lambda t\left(\mathbb{E} \mathrm{e}^{\alpha \xi}-1\right)-\alpha p t} f(x) .
$$

Substitute (5.20) into the integral in (5.18). In order to apply the dominated convergence theorem here, we notice that, by Lemma 2.26 again, there exists some $K>0$ 
such that for all $x \geq 0$ for which $f(x)>0$ and for all $t \in(0,1]$,

$$
\begin{aligned}
\frac{f(x+p t, t)}{f(x)} & =\sum_{n=1}^{\infty} \frac{(\lambda t)^{n}}{n !} \mathrm{e}^{-\lambda t} \cdot \frac{f^{n \star}(x+p t)}{f(x+p t)} \cdot \frac{f(x+p t)}{f(x)} \\
& \leq \sum_{n=1}^{\infty} \frac{(\lambda t)^{n}}{n !} \mathrm{e}^{-\lambda t} \cdot K\left(\mathbb{E} \mathrm{e}^{\alpha \xi}+1\right)^{n} \leq K \mathrm{e}^{\lambda t \mathbb{E} \mathrm{e}^{\alpha \xi}}
\end{aligned}
$$

where in the last but one step we used the local uniformity of the convergence of $f(x+y) / f(x)$ to $\mathrm{e}^{-\alpha y}$. Hence,

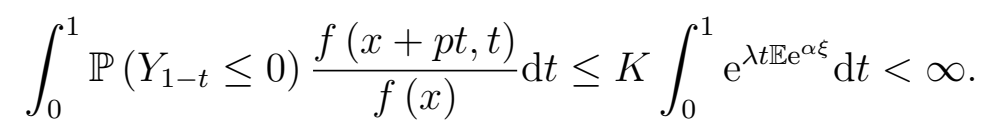

Then, using Lemma 5.8 and the dominated convergence theorem,

$$
\begin{aligned}
& \int_{0}^{1} \mathbb{P}\left(Y_{1-t} \leq 0\right) f(x+p t, t) \mathrm{d} t \\
\sim & f(x) \int_{0}^{1}\left(\frac{1}{1-t} \int_{0}^{1-t} F(p s, 1-t) \mathrm{d} s\right) \lambda t \mathrm{e}^{\lambda t\left(\mathbb{E} \mathrm{e}^{\alpha \xi}-1\right)-\alpha p t} \mathrm{~d} t .
\end{aligned}
$$

Plugging (5.19) and (5.21) into (5.18) and using the facts that $\bar{\rho}(\cdot)=\lambda \bar{F}(\cdot)$ and $f(x) / \bar{F}(x) \rightarrow \alpha$, we obtain $(5.17)$.

\subsection{The Case of Exponential-like Tails}

Finally, we consider the case that the Lévy measure $\rho$ has a light tail such that $\Pi \in \mathcal{L}(\alpha) \backslash \mathcal{S}(\alpha)$ for some $\alpha>0$.

Theorem 5.9. Consider the Lévy insurance model introduced in Section 5.1. Assume $\mathbb{E} L_{1}^{2}<\infty, \Pi \in \mathcal{L}(\alpha)$ for some $\alpha>0$, and $\bar{\Pi}(x)=o\left(\overline{\Pi^{2 *}}(x)\right)$. If $0 \leq \gamma<1$ and $0<\delta<1$ are such that condition (5.10) holds, then,

$$
\psi_{\gamma, \delta}(x) \sim \frac{1}{1-\mathbb{E} \mathrm{e}^{\alpha\left((1-\delta) L_{1}^{+}-(1-\gamma) L_{1}^{-}\right)}} \mathbb{P}\left(L_{1}>x\right)
$$


We need the following result, which is a combination of Theorem 3.3 and Corollary 6.2 of Albin and Sundén (2009):

Lemma 5.10. Let $L$ be a Lévy process with Lévy measure $\rho$ such that $\Pi \in \mathcal{L}(\alpha)$ for some $\alpha>0$ and $\bar{\Pi}(x)=o\left(\overline{\Pi^{2 *}}(x)\right)$. Then for all $t>0$, the distribution of $L_{t}$ belongs to $\mathcal{L}(\alpha) \backslash \mathcal{S}(\alpha)$ and

$$
\mathbb{P}\left(\sup _{0 \leq s \leq t} L_{s}>x\right) \sim \mathbb{P}\left(L_{t}>x\right)
$$

Note that the conditions on the Lévy measure $\rho$ in Lemma 5.10 are fulfilled if $\bar{\rho}$ is asymptotic to the tail of an exponential distribution, an Erlang distribution, or, more generally, a Gamma distribution.

Proof of Theorem 5.9. Use the notation in (5.3). By Lemma 5.10 we know that the distribution of $L_{1}$ belongs to $\mathcal{L}(\alpha) \backslash \mathcal{S}(\alpha)$ and

$$
\mathbb{P}(Y>x) \sim \mathbb{P}\left(L_{1}>x\right)
$$

Hence, the distribution of $Y$ belongs to $\mathcal{L}(\alpha) \backslash \mathcal{S}(\alpha)$ as well. Finally, using Theorem 4.1(i) again we obtain relation (5.22).

The asymptotic relation (5.22) is in terms of the tail of $L_{1}$ instead of the tail of the Lévy measure $\rho$. In case the tail of $L_{1}$ is unknown, relation (5.22) is not completely explicit. We are going to show two special, but important, cases of Theorem 5.9 in which a completely explicit asymptotic relation for the ruin probability is given.

First, we consider a gamma process $U=\left(U_{t}\right)_{t \geq 0}$ as given in Section 2.1. For the gamma process $U$, it is easy to verify that $\bar{\Pi}(x)=o\left(\overline{\Pi^{2 *}}(x)\right)$. By Theorem 5.9, we immediately have the following: 
Corollary 5.11. Consider the Lévy insurance model introduced in Section 5.1. Assume

$$
L_{t}=\Gamma_{t}-p t, \quad t \geq 0,
$$

where $p>0$ and $\Gamma=\left(\Gamma_{t}\right)_{t \geq 0}$ is a gamma process as given in Section 2.1 with parameters $\alpha, \beta>0$, i.e., $\Gamma$ starts from 0 with stationary and independent increments and $\Gamma_{1}$ has the gamma $(\alpha, \beta)$ distribution with density $f(x)=\frac{\alpha^{\beta}}{\Gamma(\beta)} x^{\beta-1} \mathrm{e}^{-\alpha x}$ for $\alpha, \beta, x>0$. If $0 \leq \gamma<1$ and $0<\delta<1$ are such that condition (5.10) holds, then,

$$
\psi_{\gamma, \delta}(x) \sim \frac{\alpha^{\beta-1}(x+p)^{\beta-1} \mathrm{e}^{-\alpha(x+p)}}{\left(1-\mathbb{E} \mathrm{e}^{\alpha\left((1-\delta) L_{1}^{+}-(1-\gamma) L_{1}^{-}\right)}\right) \Gamma(\beta)} .
$$

Next, we consider a compound Poisson process with negative drift again.

Corollary 5.12. Consider the Lévy insurance model introduced in Section 5.1. Assume

$$
L_{t}=V_{t}-p t, \quad t \geq 0
$$

where $p>0$ represents the constant premium rate and $V=\left(V_{t}\right)_{t \geq 0}$ is a compound Poisson process as given in Section 2.1. Suppose that $F$ is an exponential distribution with mean $1 / \alpha$. If $0 \leq \gamma<1$ and $0<\delta<1$ are such that condition (5.10) holds, then,

$$
\psi_{\gamma, \delta}(x) \sim \frac{2 \sqrt{\lambda / \pi}}{1-\mathbb{E} \mathrm{e}^{\alpha\left((1-\delta) L_{1}^{+}-(1-\gamma) L_{1}^{-}\right)}} \int_{0}^{\pi / 2} \Phi(\sqrt{2 \lambda} \cos \theta-\sqrt{2 \alpha(x+p)}) \mathrm{d} \theta
$$

where $\Phi(\cdot)$ is the standard normal distribution.

The following is an elementary result: 
Lemma 5.13. Let $f(\cdot):[-1,1] \rightarrow(0, \infty)$ be a nonincreasing function right continuous at 1 and let $c>0$ be a constant. Then,

$$
\int_{0}^{\pi} \mathrm{e}^{c x \cos \theta} f(\cos \theta) \mathrm{d} \theta \sim \int_{0}^{\pi / 2} \mathrm{e}^{c x \cos \theta} f(\cos \theta) \mathrm{d} \theta \sim f(1) \int_{0}^{\pi / 2} \mathrm{e}^{c x \cos \theta} \mathrm{d} \theta
$$

Proof. For every $0<\epsilon \leq \pi / 2$, we split the first integral in (5.24) into two parts as $\int_{0}^{\epsilon}+\int_{\epsilon}^{\pi}$. It is easy to see that the second part is asymptotically negligible, as

$$
\frac{\int_{\epsilon}^{\pi} \mathrm{e}^{c x \cos \theta} f(\cos \theta) \mathrm{d} \theta}{\int_{0}^{\epsilon} \mathrm{e}^{c x \cos \theta} f(\cos \theta) \mathrm{d} \theta} \leq \frac{\pi f(-1) \mathrm{e}^{c x \cos \epsilon}}{f(1) \int_{0}^{\epsilon / 2} \mathrm{e}^{c x \cos \theta} \mathrm{d} \theta} \rightarrow 0 .
$$

Hence, the first relation of (5.24) holds and, moreover,

$$
\int_{0}^{\pi / 2} \mathrm{e}^{c x \cos \theta} f(\cos \theta) \mathrm{d} \theta \sim \int_{0}^{\epsilon} \mathrm{e}^{c x \cos \theta} f(\cos \theta) \mathrm{d} \theta .
$$

Note that, since $f$ is right continuous at 1 , if in (5.25) $\epsilon>0$ is chosen to be sufficiently close to 0 , then $f(\cos \theta)$ is sufficiently close to $f(1)$. Therefore, by (5.25) and the arbitrariness of $\epsilon>0$, we obtain the second relation of (5.24).

Proof of Corollary 5.12. Clearly, $\mathbb{E} L_{1}^{2}<\infty, \Pi \in \mathcal{L}(\alpha)$, and $\bar{\Pi}(x)=o\left(\overline{\Pi^{2 *}}(x)\right)$. Therefore by Theorem 5.9, we only need to focus on derivation of the tail probability $\mathbb{P}\left(L_{1}>x\right)$. Since the $n$-fold convolution of an exponential distribution with mean $1 / \alpha$ is a gamma distribution with parameters $(\alpha, n)$, we have

$$
\begin{aligned}
\mathbb{P}\left(L_{1}>x\right) & =\int_{x+p}^{\infty} \sum_{n=1}^{\infty} \frac{\alpha^{n}}{(n-1) !} y^{n-1} \mathrm{e}^{-\alpha y} \cdot \frac{\lambda^{n}}{n !} \mathrm{e}^{-\lambda} \mathrm{d} y \\
& =\sqrt{\alpha \lambda} \mathrm{e}^{-\lambda} \int_{x+p}^{\infty} \sum_{n=0}^{\infty} \frac{(\sqrt{\alpha \lambda y})^{2 n+1}}{n !(n+1) !} y^{-1 / 2} \mathrm{e}^{-\alpha y} \mathrm{~d} y .
\end{aligned}
$$

The last series in the above is of the structure of the modified Bessel function of order 1 ; that is,

$$
\sum_{n=0}^{\infty} \frac{(\sqrt{\alpha \lambda y})^{2 n+1}}{n !(n+1) !}=\frac{1}{\pi} \int_{0}^{\pi} \mathrm{e}^{2 \sqrt{\alpha \lambda y} \cos \theta} \cos \theta \mathrm{d} \theta .
$$


Using Lemma 5.13 twice, as $y \rightarrow \infty$,

$$
\int_{0}^{\pi} \mathrm{e}^{2 \sqrt{\alpha \lambda y} \cos \theta} \cos \theta \mathrm{d} \theta \sim \int_{0}^{\pi / 2} \mathrm{e}^{2 \sqrt{\alpha \lambda y} \cos \theta} \mathrm{d} \theta \sim \mathrm{e}^{\lambda} \int_{0}^{\pi / 2} \mathrm{e}^{2 \sqrt{\alpha \lambda y} \cos \theta-\lambda \cos ^{2} \theta} \mathrm{d} \theta
$$

Substituting this into (5.27) then substituting (5.27) into (5.26), we obtain that

$$
\begin{aligned}
\mathbb{P}\left(L_{1}>x\right) & \sim \frac{\sqrt{\alpha \lambda}}{\pi} \mathrm{e}^{-\lambda} \int_{x+p}^{\infty}\left(\mathrm{e}^{\lambda} \int_{0}^{\pi / 2} \mathrm{e}^{2 \sqrt{\alpha \lambda y} \cos \theta-\lambda \cos ^{2} \theta} \mathrm{d} \theta\right) y^{-1 / 2} \mathrm{e}^{-\alpha y} \mathrm{~d} y \\
& \sim \frac{2 \sqrt{\alpha \lambda}}{\pi} \int_{0}^{\pi / 2}\left(\int_{\sqrt{x+p}}^{\infty} \mathrm{e}^{-\alpha u^{2}+2 u \sqrt{\alpha \lambda} \cos \theta-\lambda \cos ^{2} \theta} \mathrm{d} u\right) \mathrm{d} \theta \\
& =2 \sqrt{\frac{\lambda}{\pi}} \int_{0}^{\pi / 2} \Phi(\sqrt{2 \lambda} \cos \theta-\sqrt{2 \alpha(x+p)}) \mathrm{d} \theta .
\end{aligned}
$$

Substituting this into (5.22) yields (5.23). 


\section{CHAPTER 6 \\ THE RENEWAL RISK MODEL WITH RISKY INVESTMENT}

In this chapter, we study the tail behavior of the stochastically discounted net loss process in the renewal risk model with risky investment. Consider an insurance company who invests its surplus into a portfolio consisting of both a riskless bond and a risky stock. Suppose the price process of the bond grows with a constant force of interest, while the price process of the stock is modeled by an exponential Lévy process. The study of such a risk model has become a hot topic in the past decade. Paulsen (2008) gave a comprehensive review on ruin in this model when assets earn investment income. Assuming a constant mix investment strategy, i.e., the proportions of surplus invested into the riskless and risky assets remain constant, Klüppelberg and Kostadinova (2008) investigated the tail behavior of the stationary distribution for the discounted net loss process in the compound Poisson risk model. We extend their main result by deriving an asymptotic formula for the tail probability of the stochastically discounted net loss process under more general assumptions.

\subsection{Introduction}

Consider the renewal risk model in which the surplus process of an insurance company is modeled by

$$
U_{t}=u+p t-S_{t}, \quad t \geq 0
$$

where $u>0$ is the initial surplus level, $p>0$ is the constant premium rate, and $S=\left(S_{t}\right)_{t \geq 0}$ defines the aggregate claims process. Assume $S_{t}=\sum_{k=1}^{N_{t}} X_{k}, t \geq 0$, 
where, the same as in Chapter 3 , claim sizes $X_{k}, k=1,2, \ldots$, constitute a sequence of i.i.d. nonnegative random variables with generic random variable $X$ with distribution $F$ and finite mean, while their arrival times $\tau_{k}, k=1,2, \ldots$, independent of $X_{k}$, $k=1,2, \ldots$, constitute a renewal counting process

$$
N_{t}=\#\left\{k=1,2, \ldots: \tau_{k} \leq t\right\}, \quad t \geq 0
$$

Hence, the inter-arrival times $\theta_{1}=\tau_{1}, \theta_{k}=\tau_{k}-\tau_{k-1}, k=2,3, \ldots$, constitute a sequence of i.i.d., nonnegative random variables with common not-degenerate-at-zero distribution $G$, which is assumed to be nonlattice and have mean $1 / \lambda$.

Following Klüppelberg and Kostadinova (2008), suppose the insurance company is allowed to invest its surplus into two assets: a bond with constant force of interest $r>0$ and a stock whose price is modeled by an exponential Lévy process. The two assets have their price processes as, respectively,

$$
P_{t}^{(0)}=\mathrm{e}^{r t}, \quad P_{t}^{(1)}=\mathrm{e}^{L_{t}}, \quad t \geq 0
$$

where $L=\left(L_{t}\right)_{t \geq 0}$ is a Lévy process with characteristic exponent given by

$$
\Psi(s)=\mathrm{i} a s+\frac{\sigma^{2}}{2} s^{2}+\int_{-\infty}^{\infty}\left(1-\mathrm{e}^{\mathrm{i} s x}+\mathrm{i} s x 1_{\{|x| \leq 1\}}\right) \rho(\mathrm{d} x)
$$

with $a \in(-\infty, \infty), \sigma \geq 0$, and Lévy measure $\rho$ on $(-\infty, \infty)$ satisfying $\rho(\{0\})=0$ and $\int_{-\infty}^{\infty}\left(x^{2} \wedge 1\right) \rho(\mathrm{d} x)<\infty$. See Section 2.1 for more details of Lévy processes.

We assume the so-called constant mix investment strategy, i.e., the proportions of surplus invested into the bond and stock remain constant through time. The advantages of this investment strategy are discussed in Emmer et al. (2001) and 
Emmer and Klüppelberg (2004). As commented by Emmer et al. (2001, Section 2 ), this strategy is actually dynamic in the sense that it requires at every instance of time a rebalancing of the investment portfolio depending on the corresponding instantaneous price changes. From now on, we denote by $\pi \in[0,1]$ the fraction of the surplus invested into the stock.

For the two price processes in (6.1), by Lemma 2.15 we write their corresponding stochastic differential equations (SDEs) as

$$
\mathrm{d} P_{t}^{(0)}=r P_{t}^{(0)} \mathrm{d} t, \quad t \geq 0
$$

with $P_{0}^{(0)}=1$, and

$$
\begin{aligned}
\mathrm{d} P_{t}^{(1)} & =P_{t-}^{(1)} \mathrm{d} \widehat{L}_{t} \\
& =P_{t-}^{(1)}\left(\mathrm{d} L_{t}+\frac{\sigma^{2}}{2} \mathrm{~d} t+\mathrm{e}^{\Delta L_{t}}-1-\Delta L_{t}\right), \quad t>0
\end{aligned}
$$

with $P_{0}^{(1)}=1$, where $\Delta L_{t}=L_{t}-L_{t-}$ denotes the jump of the process $L$ at time $t>0$. As explained in Subsection 2.2.3, the process $\widehat{L}$ is such that $\mathrm{e}^{L_{t}}=\mathcal{E}\left(\widehat{L}_{t}\right)$, $t \geq 0$, where $\mathcal{E}$ denotes the stochastic exponential of a process. From the above two SDEs we define the investment process:

Definition 6.1. For $\pi \in[0,1]$ the investment process is defined as the solution of the SDE

$$
\mathrm{d} P_{t}^{(\pi)}=P_{t-}^{(\pi)}\left((1-\pi) r \mathrm{~d} t+\pi \mathrm{d} \widehat{L}_{t}\right), \quad t>0
$$

with $P_{0}^{(\pi)}=1$. 
Lemma 6.2 (Lemma 2.5 of Emmer and Klüppleberg (2004)). The SDE (6.2) has the solution

$$
P_{t}^{(\pi)}=\mathcal{E}\left((1-\pi) r \mathrm{~d} t+\pi \mathrm{d} \widehat{L}_{t}\right)=\mathrm{e}^{L_{\pi, t}}, \quad t \geq 0
$$

where $L_{\pi, t}$ is a Lévy process with characteristic triplet $\left(a_{\pi}, \sigma_{\pi}^{2}, \rho_{\pi}\right)$ given by

$$
\begin{aligned}
a_{\pi}= & a \pi-(1-\pi)\left(r+\frac{\sigma^{2}}{2} \pi\right) \\
& -\int_{-\infty}^{\infty}\left(\log \left(1+\pi\left(\mathrm{e}^{x}-1\right)\right) 1_{\left\{\left|\log \left(1+\pi\left(\mathrm{e}^{x}-1\right)\right)\right| \leq 1\right\}}-\pi x 1_{\{|x| \leq 1\}}\right) \rho(\mathrm{d} x), \\
\sigma_{\pi}^{2}= & \pi^{2} \sigma^{2}, \\
\rho_{\pi}(A)= & \rho\left(\left\{x: \log \left(1+\pi\left(\mathrm{e}^{x}-1\right)\right) \in A\right\}\right) \text { for any Borel set } A .
\end{aligned}
$$

In particular, when $\pi=0$ or $1, L_{\pi, t}$ reduces to $r t$ or $L_{t}$, respectively. We assume $\varphi_{\pi}(1)<\infty$ throughout this chapter so that $\mathbb{E e}^{-L_{\pi, t}}=\mathrm{e}^{t \varphi_{\pi}(1)}<\infty$ is finite for all $t \geq 0$. By Lemma 6.7 below, the assumption $\varphi_{\pi}(1)<\infty$ is implied by $\varphi(1)<\infty$.

\subsection{The Integrated Risk Process}

Suppose the insurance company invests all its surplus into the market introduced in Section 6.1 following the constant mix investment strategy. Its risk process, called integrated risk process, becomes the solution to the SDE

$$
\mathrm{d} U_{\pi, t}=p \mathrm{~d} t-\mathrm{d} S_{t}+U_{\pi, t-}\left((1-\pi) r \mathrm{~d} t+\pi \mathrm{d} \widehat{L}_{t}\right), \quad t>0
$$

with $U_{\pi, 0}=u$. The following lemma gives the solution for $\operatorname{SDE}(6.3)$. The solution can be obtained by using the method of integrating factors; see for example Bichteler (2002). For the sake of self-containedness, we still give the proof here. 
Lemma 6.3. The SDE (6.3) has the solution

$$
U_{\pi, t}=\mathrm{e}^{L_{\pi, t}}\left(u+\int_{0-}^{t} \mathrm{e}^{-L_{\pi, v}}\left(p \mathrm{~d} v-\mathrm{d} S_{v}\right)\right), \quad t \geq 0 .
$$

Proof. Define

$$
Z_{t}=\int_{0-}^{t} \mathrm{e}^{-L_{\pi, v-}}\left(p \mathrm{~d} v-\mathrm{d} S_{v}\right)=\int_{0-}^{t} \mathrm{e}^{-L_{\pi, v}}\left(p \mathrm{~d} v-\mathrm{d} S_{v}\right)
$$

where the equality holds because the processes $L_{\pi}$ and $S$ are independent and hence have no common jumps almost surely; see Proposition 5.3 of Cont and Tankov (2004). Since $P^{(\pi)}$ is an exponential Lévy process and $Z$ is the integral with respect to a finite variation process, under the moment conditions mentioned in the end of Section 6.1 both processes $P^{(\pi)}$ and $Z$ are semimartingales; see Section 2.2 for details. Hence, the integration by parts formula gives

$$
\mathrm{d}\left(P_{t}^{(\pi)} Z_{t}\right)=P_{t-}^{(\pi)} \mathrm{d} Z_{t}+Z_{t-} \mathrm{d} P_{t}^{(\pi)}+\mathrm{d}\left[P^{(\pi)}, Z\right]_{t}, \quad t>0,
$$

where $\left[P^{(\pi)}, Z\right]$ denotes the quadratic covariation process of $P^{(\pi)}$ and $Z$. Since $S$ is a finite variation process and so is $Z$ and, once again, the processes $L_{\pi}$ and $S$ have no common jumps almost surely, by the properties introduced in Subsection 2.2.2 we obtain

$$
\left[P^{(\pi)}, Z\right]_{t} \equiv 0, \quad t \geq 0
$$

Hence, for $t \geq 0$,

$$
\begin{aligned}
\mathrm{d}\left(P_{t}^{(\pi)} Z_{t}\right) & =P_{t-}^{(\pi)} \mathrm{d} Z_{t}+Z_{t-} \mathrm{d} P_{t}^{(\pi)} \\
& =P_{t-}^{(\pi)} \mathrm{e}^{-L_{\pi, t-}}\left(p \mathrm{~d} t-\mathrm{d} S_{t}\right)+\mathrm{d} P_{t}^{(\pi)} \int_{0-}^{t-} \mathrm{e}^{-L_{\pi, v}}\left(p \mathrm{~d} v-\mathrm{d} S_{v}\right) \\
& =p \mathrm{~d} t-\mathrm{d} S_{t}+\mathrm{d} P_{t}^{(\pi)} \int_{0-}^{t-} \mathrm{e}^{-L_{\pi, v}}\left(p \mathrm{~d} v-\mathrm{d} S_{v}\right)
\end{aligned}
$$


From the equality above, (6.4), and (6.2), for $t>0$,

$$
\begin{aligned}
\mathrm{d} U_{\pi, t} & =u \mathrm{~d} P_{t}^{(\pi)}+\mathrm{d}\left(P_{t}^{(\pi)} Z_{t}\right) \\
& =u \mathrm{~d} P_{t}^{(\pi)}+p \mathrm{~d} t-\mathrm{d} S_{t}+\mathrm{d} P_{t}^{(\pi)} \int_{0-}^{t-} \mathrm{e}^{-L_{\pi, v}}\left(p \mathrm{~d} v-\mathrm{d} S_{v}\right) \\
& =p \mathrm{~d} t-\mathrm{d} S_{t}+P_{t-}^{(\pi)}\left(u+\int_{0-}^{t-} \mathrm{e}^{-L_{\pi, v}}\left(p \mathrm{~d} v-\mathrm{d} S_{v}\right)\right) \frac{\mathrm{d} P_{t}^{(\pi)}}{P_{t-}^{(\pi)}} \\
& =p \mathrm{~d} t-\mathrm{d} S_{t}+U_{\pi, t-}\left((1-\pi) r \mathrm{~d} t+\pi \mathrm{d} \widehat{L}_{t}\right) .
\end{aligned}
$$

This verifies (6.3).

\subsection{The Discounted Net Loss Process}

Definition 6.4. The discounted net loss process is defined as

$$
V_{\pi, t}=u-\mathrm{e}^{-L_{\pi, t}} U_{\pi, t}=\int_{0-}^{t} \mathrm{e}^{-L_{\pi, v}}\left(\mathrm{~d} S_{v}-p \mathrm{~d} v\right), \quad t \geq 0
$$

where $U_{\pi, t}$ is given in (6.4).

We are interested in the tail behavior of the stationary discounted net loss process. We study $V_{\pi}$ via its natural discretization at claim-arrival times, $V_{\pi, \tau_{k}}, k=$ $0,1, \ldots$, where $\tau_{0}=0$. For $k=1,2, \ldots$, write

$$
\begin{aligned}
& A_{\pi, k}=X_{k} \mathrm{e}^{-\left(L_{\pi, \tau_{k}}-L_{\pi, \tau_{k-1}}\right)}-p \int_{\tau_{k-1}}^{\tau_{k}} \mathrm{e}^{-\left(L_{\pi, v}-L_{\pi, \tau_{k-1}}\right)} \mathrm{d} v \\
& B_{\pi, k}=\mathrm{e}^{-\left(L_{\pi, \tau_{k}}-L_{\pi, \tau_{k-1}}\right)}
\end{aligned}
$$

Then $\left(A_{\pi, k}, B_{\pi, k}\right), k=1,2, \ldots$, form a sequence of i.i.d. random pairs with generic random pair

$$
\left(A_{\pi}, B_{\pi}\right)=\left(X \mathrm{e}^{-L_{\pi, \theta}}-p \int_{0}^{\theta} \mathrm{e}^{-L_{\pi, v}} \mathrm{~d} v, \mathrm{e}^{-L_{\pi, \theta}}\right) .
$$


It is obvious that

$$
V_{\pi, 0}=0, \quad V_{\pi, \tau_{k}}=\sum_{m=1}^{k} A_{\pi, m} \prod_{j=1}^{m-1} B_{\pi, j}, \quad k=1,2, \ldots,
$$

where the product over an empty set of indices produces a value 1.

Denoting by $-\eta$ the left abscissa of convergence of $g(s)=\mathbb{E} \mathrm{e}^{-s \theta}$, we give the following theorem:

Theorem 6.5. Consider the renewal risk model with risky investment introduced in Section 6.1. Suppose $\mathbb{E} X<\infty, \mathbb{E} L_{1}>0$, and the Laplace exponent of $L_{\pi}$ satisfies $\varphi_{\pi}(1)<\eta$

(i) We have

$$
V_{\pi, \tau_{k}} \stackrel{\text { a.s. }}{\longrightarrow} V_{\pi, \infty}=\sum_{m=1}^{\infty} A_{\pi, m} \prod_{j=1}^{m-1} B_{\pi, j}, \quad \text { as } k \rightarrow \infty,
$$

where the series of the right-hand side converges absolutely with probability 1. Moreover, $V_{\pi, \infty}$ satisfies the stochastic difference equation

$$
V_{\pi, \infty} \stackrel{d}{=} A_{\pi}+B_{\pi} V_{\pi, \infty}
$$

where $V_{\pi, \infty}$ and $\left(A_{\pi}, B_{\pi}\right)$ are independent.

(ii) $V_{\pi, t}$ almost surely converges to some finite random variable $V_{\pi, \infty}^{c}$ if and only if $V_{\pi, \tau_{k}}$ almost surely converges to some finite random variable and $V_{\pi, \infty}$. Furthermore,

$$
V_{\pi, \infty} \stackrel{a . s .}{=} V_{\pi, \infty}^{c}
$$

To give the proof of Theorem 6.5, we need some lemmas. The following lemma, which was proved by Ross (1983), holds for a general renewal counting process: 
Lemma 6.6 (Proposition 3.4.5 of Ross (1983)). Consider the renewal counting process $\left(N_{t}\right)_{t \geq 0}$ given in (3.1) whose i.i.d. inter-arrival times follow a common non-lattice distribution $G$ with finite mean. As $t \rightarrow \infty, t-\tau_{N_{t}}$ converges in distribution to $G_{e}$, the equilibrium distribution of $G$.

Lemma 6.7 (Lemma A.1 of Klüppelberg and Kostadinova (2008)). Consider L and $L_{\pi}$ introduced above. We have

(i) If $\mathbb{E} L_{1}<\infty$, then $\mathbb{E} L_{\pi, 1}<\infty$.

(ii) If $\mathbb{E} L_{1}>0$, then $\mathbb{E} L_{\pi, 1}>0$.

(iii) If $\varphi(s)=\log \mathbb{E} \mathrm{e}^{-s L_{1}}<\infty$, then $\varphi_{\pi}(s)=\log \mathbb{E}^{-s L_{\pi, 1}}<\infty$.

For $a>0$, denote $\log ^{+} a=\max \{0, \log a\}$. We have the following lemma:

Lemma 6.8. Assume $\mathbb{E} X<\infty, \mathbb{E} L_{1}>0$, and $\varphi_{\pi}(1)<\eta$. Then for $A_{\pi}$ and $B_{\pi}$ defined in (6.6), we have

$$
\mathbb{E} \log ^{+}\left|A_{\pi}\right|<\infty \quad \text { and } \quad \mathbb{E} \log B_{\pi}<0 \text {. }
$$

Proof. By Lemma 6.7(i), we have $0<\mathbb{E} L_{\pi, 1}<\infty$. It is clear that

$$
\mathbb{E} \log B_{\pi}=-\mathbb{E} L_{\pi, \theta}=-\mathbb{E} L_{\pi, 1} \mathbb{E} \theta<0
$$

For the proof of $\mathbb{E} \log ^{+}\left|A_{\pi}\right|<\infty$, we use the elementary inequality $\log x<x$ for all $x>0$. Then,

$$
\begin{aligned}
\mathbb{E} \log ^{+}\left|A_{\pi}\right| & =\mathbb{E} \log ^{+}\left|X \mathrm{e}^{-L_{\pi, \theta}}-p \int_{0}^{\theta} \mathrm{e}^{-L_{\pi, v}} \mathrm{~d} v\right| \\
& \leq \mathbb{E}\left|X \mathrm{e}^{-L_{\pi, \theta}}-p \int_{0}^{\theta} \mathrm{e}^{-L_{\pi, v}} \mathrm{~d} v\right| \\
& \leq \mathbb{E} X \mathbb{E}^{-L_{\pi, \theta}}+p \mathbb{E} \int_{0}^{\theta} \mathrm{e}^{-L_{\pi, v}} \mathrm{~d} v
\end{aligned}
$$


In the first term, $\mathbb{E} X<\infty$ and, since $\varphi_{\pi}(1)<\eta$,

$$
\mathbb{E} \mathrm{e}^{-L_{\pi, \theta}}=\int_{0-}^{\infty} \mathbb{E} \mathrm{e}^{-L_{\pi, v}} G(\mathrm{~d} v)=\int_{0-}^{\infty} \mathrm{e}^{v \varphi_{\pi}(1)} G(\mathrm{~d} v)<\infty
$$

In the second term, using $\varphi_{\pi}(1)<\eta$ again,

$$
\begin{aligned}
\mathbb{E} \int_{0}^{\theta} \mathrm{e}^{-L_{\pi, v}} \mathrm{~d} v & =\int_{0-}^{\infty} \int_{0}^{t} \mathbb{E} \mathrm{e}^{-L_{\pi, v}} \mathrm{~d} v G(\mathrm{~d} v)=\int_{0-}^{\infty} \int_{0}^{t} \mathrm{e}^{v \varphi_{\pi}(1)} \mathrm{d} v G(\mathrm{~d} t) \\
& = \begin{cases}\int_{0-}^{\infty} t G(\mathrm{~d} t)=\mathbb{E} \theta=1 / \lambda<\infty & \text { if } \varphi_{\pi}(1)=0 \\
\frac{1}{\varphi_{\pi}(1)}\left(\int_{0-}^{\infty} \mathrm{e}^{t \varphi_{\pi}(1)} G(\mathrm{~d} t)-1\right)<\infty & \text { if } \varphi_{\pi}(1) \neq 0\end{cases}
\end{aligned}
$$

This ends the proof.

Now we are ready to give the proof of Theorem 6.5 .

Proof of Theorem 6.5. (i) In order to prove (6.7) and (6.8) we introduce random variables $\widehat{V}_{\pi, \tau_{k}}, k=0,1, \ldots$, such that $\widehat{V}_{\pi, 0}=0$ and

$$
\widehat{V}_{\pi, \tau_{k}}=A_{\pi, k}+B_{\pi, k} \widehat{V}_{\pi, \tau_{k-1}}^{k}=\sum_{m=1}^{k} A_{\pi, m} \prod_{j=m+1}^{k} B_{\pi, j}, \quad k=1,2, \ldots
$$

We observe that for every $k=1,2, \ldots$,

$$
\left\{\left(A_{\pi, j}, B_{\pi, j}\right), j=1,2, \ldots k\right\} \stackrel{d}{=}\left\{\left(A_{\pi, k-j+1}, B_{\pi, k-j+1}\right), j=1,2, \ldots k\right\}
$$

which implies that

$$
\sum_{m=1}^{k} A_{\pi, m} \prod_{j=1}^{m-1} B_{\pi, j} \stackrel{d}{=} \sum_{m=1}^{k} A_{\pi, m} \prod_{j=m+1}^{k} B_{\pi, j}
$$

Hence, $V_{\pi, \tau_{k}} \stackrel{\mathrm{d}}{=} \widehat{V}_{\pi, \tau_{k}}$ holds for every $k=1,2, \ldots$. Applying Proposition 8.4.3 of Embrechts et al. (1997) on $\widehat{V}_{\pi, \tau_{k}}$ we obtain (6.7) and (6.8) immediately; see also Vervaat (1979). The conditions in that proposition are guaranteed by Lemma 6.8. 
(ii) For every $t \geq 0$,

$$
V_{\pi, t}=V_{\pi, \tau_{N_{t}}}-p \mathrm{e}^{-L_{\pi, \tau_{N_{t}}}} \int_{\tau_{N_{t}}}^{t} \mathrm{e}^{-\left(L_{\pi, v}-L_{\pi, \tau_{N}}\right)} \mathrm{d} v
$$

where in the last line the integral is independent of $V_{\pi, \tau_{N_{t}}}$. Since $N_{t} \stackrel{\text { a.s. }}{\rightarrow} \infty$ as $t \rightarrow \infty$,

we know from (i) that $V_{\pi, \tau_{N_{t}}} \stackrel{\text { a.s. }}{\rightarrow} V_{\pi, \infty}$ as $t \rightarrow \infty$. Moreover, as $\mathbb{E} L_{1}>0$, by Lemma 6.7 (ii) we have that $\mathbb{E} L_{\pi, 1}>0$ and hence $\mathrm{e}^{-L_{\pi, \tau}}$ a.s. 0 as $t \rightarrow \infty$. Finally,

$$
\int_{\tau_{N_{t}}}^{t} \mathrm{e}^{-\left(L_{\pi, v}-L_{\pi, \tau_{N_{t}}}\right)} \mathrm{d} v \stackrel{d}{=} \int_{0}^{t-\tau_{N_{t}}} \mathrm{e}^{-L_{\pi, v}} \mathrm{~d} v
$$

As $t-\tau_{N_{t}}$ converges in distribution to $G_{e}$ as $t \rightarrow \infty$ by Lemma 6.6 , the last integral almost surely converges to a finite random variable. Then relation (6.9) follows immediately.

\subsection{Claims with Extended-regularly-varying Tails}

In this section, we assume that $F \in \mathrm{ERV}$. Recalling $\lambda_{t}=\mathbb{E} N_{t}$ and $\Lambda=\{t$ : $\left.\lambda_{t}>0\right\} \cup\{\infty\}$, we give an explicit expression for the asymptotic tail probability of $V_{\pi, T}$ for all $T \in \Lambda$ in the following theorem:

Theorem 6.9. Consider $V_{\pi, t}$ defined in (6.5). Suppose $F \in \operatorname{ERV}(-\alpha,-\beta)$ for some $0<\alpha \leq \beta<\infty, \mathbb{E} L_{1}>0$, and $\varphi_{\pi}(\beta+\varepsilon)<0$ for some $\varepsilon>0$. Then, it holds for every $T \in \Lambda$ that

$$
\mathbb{P}\left(V_{\pi, T}>x\right) \sim \int_{0-}^{T} \bar{F}\left(x \mathrm{e}^{L_{\pi, t}}\right) \mathrm{d} \lambda_{t} .
$$

When $\alpha=\beta$ and $T=\infty$, it can be derived from relation (6.10) that

$$
\mathbb{P}\left(V_{\pi, \infty}>x\right) \sim \frac{\mathbb{E} \mathrm{e}^{\varphi_{\pi}(\alpha) \theta}}{1-\mathbb{E} \mathrm{e}^{\varphi_{\pi}(\alpha) \theta}} \bar{F}(x) .
$$


Klüppelberg and Kostadinova (2008) obtained relation (6.11) for the special case that $\left(N_{t}\right)_{t \geq 0}$ is a Poisson process by applying a key result of Grey (1994); see Theorem 4.6(a) of Klüppelberg and Kostadinova (2008). See also Heyde and Wang (2009) for a result of the finite-time ruin probability similar to $(6.11)$ but for $\left(N_{t}\right)_{t \geq 0}$ being a Poisson process.

To prove Theorem 6.9, we first prepare two lemmas. The following lemma was obtained by Wang and Tang (2006):

Lemma 6.10. Let $\left\{X_{k}, k=1,2, \ldots\right\}$ be a sequence of i.i.d. nonnegative random variables with common distribution $F$ on $[0, \infty)$ and $\left\{\omega_{k}, k=1,2, \ldots\right\}$ another sequence of positive random variables. Suppose the two sequences are mutually independent. If $F \in \operatorname{ERV}(-\alpha,-\beta)$ for some $0<\alpha \leq \beta<\infty$ and

$$
\mathbb{E}\left(\sum_{k=1}^{\infty} \omega_{k}^{u}\right)^{v}<\infty
$$

for some $0<u<\min \{1, \alpha\}$ and $v>\beta / u$. Then, it holds that

$$
\mathbb{P}\left(\sum_{k=1}^{\infty} \omega_{k} X_{k}>x\right) \sim \sum_{k=1}^{\infty} \mathbb{P}\left(\omega_{k} X_{k}>x\right)
$$

The next lemma, obtained by Maulik and Zwart (2006), concerns the exponential functional of a Lévy process:

Lemma 6.11. Let $\left(L_{t}\right)_{t \geq 0}$ be a Lévy process with Laplace exponent $\varphi(\cdot)$ and $W=$ $\int_{0}^{\infty} \mathrm{e}^{-L_{t}} \mathrm{~d} t$

(i) $W<\infty$ almost surely if and only if $L_{t} \stackrel{a . s .}{\rightarrow} \infty$ as $t \rightarrow \infty$;

(ii) If $s>0$ and $\varphi(s)<0$, then $\mathbb{E} W^{s}<\infty$. 
Now we are ready to give the proof of Theorem 6.9 .

Proof of Theorem 6.9. Throughout this proof, $0<t \leq T$ is understood as $0<$ $t<\infty$ when $T=\infty$.

First we derive an upper asymptotic bound for $\mathbb{P}\left(V_{\pi, T}>x\right)$. It is clear from the definition of $V_{\pi, T}$ in (6.5) that

$$
\mathbb{P}\left(V_{\pi, T}>x\right) \leq \mathbb{P}\left(\sum_{k=1}^{\infty} X_{k} \mathrm{e}^{-L_{\pi, \tau_{k}}} 1_{\left(\tau_{k} \leq T\right)}>x\right)
$$

To apply Lemma 6.10 , we see that, for all $u$ and $v$ such that $0<u<\min \{1, \alpha\}$ and $\beta<u v<\beta+\varepsilon$

$$
\mathbb{E}\left(\sum_{k=1}^{\infty} \mathrm{e}^{-u L_{\pi, \tau_{k}}} 1_{\left(\tau_{k} \leq T\right)}\right)^{v} \leq \mathbb{E}\left(\sum_{k=1}^{\infty} c k^{-2} c^{-1} k^{2} \mathrm{e}^{-u L_{\pi, \tau_{k}}}\right)^{v}
$$

where $c$ is the constant such that $c \sum_{k=1}^{\infty} k^{-2}=1$. By Jensen's inequality, it holds almost surely that

$$
\left(\sum_{k=1}^{\infty} c k^{-2} c^{-1} k^{2} \mathrm{e}^{-u L_{\pi, \tau_{k}}}\right)^{v} \leq \sum_{k=1}^{\infty} c k^{-2}\left(c^{-1} k^{2} \mathrm{e}^{-u L_{\pi, \tau_{k}}}\right)^{v} .
$$

Since $\varphi_{\pi}(0)=0, \varphi_{\pi}(\beta+\varepsilon)<0$, and $\varphi_{\pi}(\cdot)$ is strictly convex, $\varphi_{\pi}(s)<0$ for all $s \in(0, \beta+\varepsilon]$. Following (6.12), we have

$$
\begin{aligned}
\mathbb{E}\left(\sum_{k=1}^{\infty} \mathrm{e}^{-u L_{\pi, \tau_{k}}} 1_{\left(\tau_{k} \leq T\right)}\right)^{v} & \leq c^{1-v} \sum_{k=1}^{\infty} k^{2 v-2} \mathbb{E}^{-u v L_{\pi, \tau_{k}}} \\
& =c^{1-v} \sum_{k=1}^{\infty} k^{2 v-2} \mathrm{e}^{\varphi_{\pi}(u v) k}<\infty
\end{aligned}
$$

Therefore, by Lemma 6.10,

$$
\mathbb{P}\left(V_{\pi, T}>x\right) \lesssim \sum_{k=1}^{\infty} \mathbb{P}\left(X_{k} \mathrm{e}^{-L_{\pi, \tau_{k}}} 1_{\left(\tau_{k} \leq T\right)}>x\right)=\int_{0-}^{T} \bar{F}\left(x \mathrm{e}^{L_{\pi, t}}\right) \mathrm{d} \lambda_{t} .
$$


Then we derive the corresponding lower asymptotic bound for $\mathbb{P}\left(V_{\pi, T}>x\right)$.

Since $\mathbb{E} L_{1}>0$, by Lemmas 6.7(ii) and 6.11(i) we know that $W_{\pi}=\int_{0}^{\infty} \mathrm{e}^{-L_{\pi, t}} \mathrm{~d} t$ is a finite random variable. Likewise, from (6.5) we have

$$
\mathbb{P}\left(V_{\pi, T}>x\right) \geq \mathbb{P}\left(\sum_{k=1}^{\infty} X_{k} \mathrm{e}^{-L_{\pi, \tau_{k}}} 1_{\left(\tau_{k} \leq T\right)}>x+p W_{\pi}\right) .
$$

For arbitrarily fixed $\varepsilon_{1}>0$, it holds that

$$
\begin{aligned}
\mathbb{P}\left(V_{\pi, T}>x\right) & \geq \mathbb{P}\left(\sum_{k=1}^{\infty} X_{k} \mathrm{e}^{-L_{\pi, \tau_{k}}} 1_{\left(\tau_{k} \leq T\right)}>\left(1+\varepsilon_{1}\right) x\right)-\mathbb{P}\left(p W_{\pi}>\varepsilon_{1} x\right) \\
& =I_{1}(x)-I_{2}(x) .
\end{aligned}
$$

Similarly to the above,

$$
I_{1}(x) \sim \int_{0}^{T} \bar{F}\left(\left(1+\varepsilon_{1}\right) x \mathrm{e}^{L_{\pi, t}}\right) \mathrm{d} \lambda_{t} .
$$

Arbitrarily choose some $\varepsilon_{2}>0$ such that the inequality

$$
\frac{\bar{F}\left(\left(1+\varepsilon_{1}\right) x\right)}{\bar{F}(x)} \geq\left(1+\varepsilon_{1}\right)^{-\beta-1}
$$

holds for all $x>1 / \varepsilon_{2}$. Then, uniformly for all $t>0$,

$$
\begin{aligned}
\bar{F}\left(\left(1+\varepsilon_{1}\right) x \mathrm{e}^{L_{\pi, t}}\right) & \geq \mathbb{P}\left(X \mathrm{e}^{-L_{\pi, t}}>\left(1+\varepsilon_{1}\right) x, \mathrm{e}^{-L_{\pi, t}} \leq \varepsilon_{2} x\right) \\
& \gtrsim\left(1+\varepsilon_{1}\right)^{-\beta-1} \mathbb{P}\left(X \mathrm{e}^{-L_{\pi, t}}>x, \mathrm{e}^{-L_{\pi, t}} \leq \varepsilon_{2} x\right) \\
& \geq\left(1+\varepsilon_{1}\right)^{-\beta-1}\left(\bar{F}\left(x \mathrm{e}^{L_{\pi, t}}\right)-\mathbb{P}\left(\mathrm{e}^{-L_{\pi, t}}>\varepsilon_{2} x\right)\right) \\
& \geq\left(1+\varepsilon_{1}\right)^{-\beta-1}\left(\bar{F}\left(x \mathrm{e}^{L_{\pi, t}}\right)-\frac{\mathrm{e}^{\varphi_{\pi}(\beta+\varepsilon) t}}{\left(\varepsilon_{2} x\right)^{\beta+\varepsilon}}\right) .
\end{aligned}
$$

It follows from (6.14) that

$$
\begin{aligned}
I_{1}(x) & \gtrsim\left(1+\varepsilon_{1}\right)^{-\beta-1}\left(\int_{0}^{T} \bar{F}\left(x \mathrm{e}^{L_{\pi, t}}\right) \mathrm{d} \lambda_{t}-\int_{0}^{T} \frac{\mathrm{e}^{\varphi_{\pi}(\beta+\varepsilon) t}}{\left(\varepsilon_{2} x\right)^{\beta+\varepsilon}} \mathrm{d} \lambda_{t}\right) \\
& =\left(1+\varepsilon_{1}\right)^{-\beta-1} \int_{0}^{T} \bar{F}\left(x \mathrm{e}^{L_{\pi, t}}\right) \mathrm{d} \lambda_{t}-o(\bar{F}(x))
\end{aligned}
$$


By Lemma 6.11(ii), $\mathbb{E} W_{\pi}^{\beta+\varepsilon}<\infty$. Hence,

$$
I_{2}(x)=o(\bar{F}(x))
$$

Substitute these estimates into (6.13) to obtain that

$$
\mathbb{P}\left(V_{\pi, T}>x\right) \gtrsim\left(1+\varepsilon_{1}\right)^{-\beta-1} \int_{0}^{T} \bar{F}\left(x \mathrm{e}^{L_{\pi, t}}\right) \mathrm{d} \lambda_{t}-o(\bar{F}(x))
$$

Since it holds for arbitrarily fixed $M>0$ that

$$
\begin{aligned}
\int_{0}^{T} \bar{F}\left(x \mathrm{e}^{L_{\pi, t}}\right) \mathrm{d} \lambda_{t} & \geq \int_{0}^{T} \mathbb{P}\left(X \mathrm{e}^{-L_{\pi, t}}>x, \sup _{0<t \leq T} L_{\pi, t} \leq M\right) \mathrm{d} \lambda_{t} \\
& \geq \lambda_{T} \bar{F}\left(x \mathrm{e}^{M}\right) \mathbb{P}\left(\sup _{0<t \leq T} L_{\pi, t} \leq M\right) \\
& \asymp \bar{F}(x),
\end{aligned}
$$

where $a(x) \asymp b(x)$ means that $\limsup _{x \rightarrow \infty} a(x) / b(x)<\infty$ and $\lim \sup _{x \rightarrow \infty} b(x) / a(x)<$ $\infty$, it follows that

$$
\mathbb{P}\left(V_{\pi, T}>x\right) \gtrsim\left(1+\varepsilon_{1}\right)^{-\beta-1} \int_{0}^{T} \bar{F}\left(x \mathrm{e}^{L_{\pi, t}}\right) \mathrm{d} \lambda_{t} .
$$

Since $\varepsilon_{1}>0$ can be arbitrarily small, it follows that

$$
\mathbb{P}\left(V_{\pi, T}>x\right) \gtrsim \int_{0}^{T} \bar{F}\left(x \mathrm{e}^{L_{\pi, t}}\right) \mathrm{d} \lambda_{t} .
$$

This ends the proof of Theorem 6.9. 


\section{REFERENCES}

Albin, J. M. P.; Sundén, M. On the asymptotic behaviour of Lévy processes. Part I: Subexponential and exponential processes. Stochastic Process. Appl. 119 (2009), no. 1, 281-304.

Albrecher, H.; Badescu, A.; Landriault, D. On the dual risk model with tax payments. Insurance Math. Econom. 42 (2008a), no. 3, 1086-1094.

Albrecher, H.; Borst, S.; Boxma, O.; Resing, J. The tax identity in risk theory - a simple proof and an extension. Insurance Math. Econom. 44 (2009), no. 2, 304-306.

Albrecher, H.; Hipp, C. Lundberg's risk process with tax. Bl. DGVFM 28 (2007), no. $1,13-28$.

Albrecher, H.; Renaud, J.-F.; Zhou, X. A Lévy insurance risk process with tax. J. Appl. Probab. 45 (2008b), no. 2, 363-375.

Araman, V. F.; Glynn, P. W. Tail asymptotics for the maximum of perturbed random walk. Ann. Appl. Probab. 16 (2006), no. 3, 1411-1431.

Asmussen, S. Ruin probabilities. World Scientific Publishing Co., Inc., River Edge, NJ, 2000.

Asmussen, S. Applied probability and queues. Second edition. Springer-Verlag, New York, 2003.

Asmussen, S.; Schmidli, H.; Schmidt, V. Tail probabilities for non-standard risk and queueing processes with subexponential jumps. Adv. in Appl. Probab. 31 (1999), no. 2, 422-447.

Bachelier, L. Théorie de la spéculation. Ann. Sci. École Norm. Sup. III-17 (1900), 21-86. Translated in: Cootner, P. H. The Random Character of Stock Market Prices, pp. 17-78. MIT Press, Cambridge, Mass., 1964.

Bichteler, K. Stochastic integration with jumps. Cambridge University Press, Cambridge, 2002.

Bingham, N. H.; Goldie, C. M.; Teugels, J. L. Regular variation. Cambridge University Press, Cambridge, 1987. 
Braverman, M. Suprema and sojourn times of Lévy processes with exponential tails. Stochastic Process. Appl. 68 (1997), no. 2, 265-283.

Braverman, M.; Samorodnitsky, G. Functionals of infinitely divisible stochastic processes with exponential tails. Stochastic Process. Appl. 56 (1995), no. 2, 207-231.

Cai, J.; Kalashnikov, V. NWU property of a class of random sums. J. Appl. Probab. 37 (2000), no. 1, 283-289.

Chen, Y.; Ng, K. W.; Tang, Q. Weighted sums of subexponential random variables and their maxima. Adv. in Appl. Probab. 37 (2005), no. 2, 510-522.

Chhikara, R. S.; Folks, J. L. The inverse Gaussian distribution: theory, methodology, and applications. Marcel Dekker, Inc., New York, 1989.

Chover, J.; Ney, P.; Wainger, S. Functions of probability measures. J. Analyse Math. 26 (1973), 255-302.

Cline, D. B. H. Convolution tails, product tails and domains of attraction. Probab. Theory Relat. Fields 72 (1986), no. 4, 529-557.

Cline, D. B. H.; Samorodnitsky, G. Subexponentiality of the product of independent random variables. Stochastic Process. Appl. 49 (1994), no. 1, 75-98.

Cont, R.; Tankov, P. Financial modelling with jump processes. Chapman \& Hall/CRC, Boca Raton, FL, 2004.

Doney, R. A.; Kyprianou, A. E. Overshoots and undershoots of Lévy processes. Ann. Appl. Probab. 16 (2006), no. 1, 91-106.

Dufresne, D. The distribution of a perpetuity, with applications to risk theory and pension funding. Scand. Actuar. J. (1990), no. 1-2, 39-79.

Embrechts, P. A property of the generalized inverse Gaussian distribution with some applications. J. Appl. Probab. 20 (1983), no. 3, 537-544.

Embrechts, P.; Goldie, C. M. On closure and factorization properties of subexponential and related distributions. J. Austral. Math. Soc. Ser. A 29 (1980), no. $2,243-256$.

Embrechts, P.; Goldie, C. M. On convolution tails. Stochastic Process. Appl. 13 (1982), no. 3, 263-278. 
Embrechts, P.; Klüppelberg, C.; Mikosch, T. Modelling extremal events for insurance and finance. Springer-Verlag, Berlin, 1997.

Embrechts, P.; Omey, E. A property of longtailed distributions. J. Appl. Probab. 21 (1984), no. 1, 80-87.

Emmer, S.; Klüppelberg, C. Optimal portfolios when stock prices follow an exponential Lévy process. Finance Stoch. 8 (2004), no. 1, 17-44.

Emmer, S.; Klüppelberg, C.; Korn, R. Optimal portfolios with bounded capital at risk. Math. Finance 11 (2001), no. 4, 365-384.

Esary, J. D.; Proschan, F.; Walkup, D. W. Association of random variables, with applications. Ann. Math. Statist. 38 (1967), 1466-1474.

Feller, W. An introduction to probability theory and its applications. Vol. II. John Wiley \& Sons, Inc., New York-London-Sydney, 1966.

Gjessing, H. K.; Paulsen, J. Present value distributions with applications to ruin theory and stochastic equations. Stochastic Process. Appl. 71 (1997), no. 1, $123-144$.

Goll, T.; Kallsen, J. Optimal portfolios for logarithmic utility. Stochastic Process. Appl. 89 (2000), no. 1, 31-48.

Hao, X.; Tang, Q. A uniform asymptotic estimate for discounted aggregate claims with subexponential tails. Insurance Math. Econom. 43 (2008), no. 1, 116-120.

Hao, X.; Tang, Q. Asymptotic ruin probabilities of the Lévy insurance model under periodic taxation. Submitted to Astin Bull., 2009.

Hao, X.; Tang, Q.; Wei, L. On the maximum exceedance of a sequence of random variables over a renewal threshold. J. Appl. Probab. 46 (2009), no. 2, 1-12.

Heyde, C. C.; Wang, D. Finite-time ruin probability with an exponential Lévy process investment return and heavy-tailed claims. Adv. in Appl. Probab. 41 (2009), no. 1, 206-224.

Johnson, M. E. Multivariate statistical simulations. Wiley, New York, 1987.

Klebaner, F. C. Introduction to stochastic calculus with applications. Second edition. Imperial College Press, London, 2005. 
Klüppelberg, C. Subexponential distributions and integrated tails. J. Appl. Probab. 25 (1988), no. 1, 132-141.

Klüppelberg, C. Subexponential distributions and characterizations of related classes. Probab. Theory Related Fields 82 (1989), no. 2, 259-269.

Klüppelberg, C.; Kostadinova, R. Integrated insurance risk models with exponential Lévy investment. Insurance Math. Econom. 42 (2008), no. 2, 560-577.

Klüppelberg, C.; Kyprianou, A. E.; Maller, R. A. Ruin probabilities and overshoots for general Lévy insurance risk processes. Ann. Appl. Probab. 14 (2004), no. 4, 1766-1801.

Konstantinides, D.; Tang, Q. The probabilities of absolute ruin in the renewal risk model with constant force of interest. 2009, working paper.

Konstantinides, D.; Tang, Q.; Tsitsiashvili, G. Estimates for the ruin probability in the classical risk model with constant interest force in the presence of heavy tails. Insurance Math. Econom. 31 (2002), no. 3, 447-460.

Kyprianou, A. E. Introductory lectures on fluctuations of Lévy processes with applications. Springer-Verlag, Berlin, 2006.

Lundberg, F. Approximerad framställning av sannolikhetsfunktionen. Återförsäkring av kollektivrisker. Akad. Afhandling. Almqvist och Wiksell, Uppsala, 1903.

Maulik, K.; Zwart, B. Tail asymptotics for exponential functionals of Lévy processes. Stochastic Process. Appl. 116 (2006), no. 2, 156-177.

Nilsen, T.; Paulsen, J. On the distribution of a randomly discounted compound Poisson process. Stochastic Process. Appl. 61 (1996), no. 2, 305-310.

Pakes, A. G. Convolution equivalence and infinite divisibility. J. Appl. Probab. 41 (2004), no. 2, 407-424.

Palmowski, Z.; Zwart, B. Tail asymptotics of the supremum of a regenerative process. J. Appl. Probab. 44 (2007), no. 2, 349-365.

Paulsen, J. Risk theory in a stochastic economic environment. Stochastic Process. Appl. 46 (1993), no. 2, 327-361.

Paulsen, J. Present value of some insurance portfolios. Scand. Actuar. J. (1997), no. $1,11-37$. 
Paulsen, J. Ruin models with investment income. Probab. Surv. 5 (2008), 416-434.

Prabhu, N. U. Stochastic processes. Basic theory and its applications. The Macmillan Co., New York, 1965.

Protter, P. E. Stochastic integration and differential equations. Second edition. Version 2.1. Corrected third printing. Springer-Verlag, Berlin, 2005.

Robert, C. Y. Asymptotic probabilities of an exceedance over renewal thresholds with an application to risk theory. J. Appl. Probab. 42 (2005), no. 1, 153-162.

Rogozin, B. A. On the constant in the definition of subexponential distributions. Theory Probab. Appl. 44 (2000), no. 2, 409-412.

Rogozin, B. A.; Sgibnev, M. S. Banach algebras of measures on the line with given asymptotics of distributions at infinity. Siberian Math. J. 40 (1999), no. 3, $565-576$.

Rootzén, H.; Tajvidi, N. Extreme value statistics and wind storm losses: a case study. Scand. Actuar. J. (1997), no. 1, 70-94.

Rosiński, J.; Samorodnitsky, G. Distributions of subadditive functionals of sample paths of infinitely divisible processes. Ann. Probab. 21 (1993), no. 2, 9961014 .

Ross, S. M. Stochastic processes. John Wiley \& Sons, Inc., New York, 1983.

Seshadri, V. The inverse Gaussian distribution. Statistical theory and applications. Springer-Verlag, New York, 1999.

Stein, C. A note on cumulative sums. Ann. Math. Statistics 17 (1946), 498-499.

Su, C.; Tang, Q. Characterizations on heavy-tailed distributions by means of hazard rate. Acta Math. Appl. Sin. Engl. Ser. 19 (2003), no. 1, 135-142.

Tang, Q. The finite-time ruin probability of the compound Poisson model with constant interest force. J. Appl. Probab. 42 (2005), no. 3, 608-619.

Tang, Q. The overshoot of a random walk with negative drift. Statist. Probab. Lett. 77 (2007a), no. 2, 158-165.

Tang, Q. Heavy tails of discounted aggregate claims in the continuous-time renewal model. J. Appl. Probab. 44 (2007b), no. 2, 285-294. 
Tang, Q.; Tsitsiashvili, G. Randomly weighted sums of subexponential random variables with application to ruin theory. Extremes 6 (2003a), no. 3, 171-188.

Tang, Q.; Tsitsiashvili, G. Precise estimates for the ruin probability in finite horizon in a discrete-time model with heavy-tailed insurance and financial risks. Stochastic Process. Appl. 108 (2003b), no. 2, 299-325.

Tang, Q.; Wang, G.; Yuen, K. C. Uniform tail asymptotics for stochastically discounted aggregate claims in the renewal model. 2009, working paper.

Veraverbeke, N. Asymptotic behaviour of Wiener-Hopf factors of a random walk. Stochastic Processes Appl. 5 (1977), no. 1, 27-37.

Vervaat, W. On a stochastic difference equation and a representation of nonnegative infinitely divisible random variables. Adv. in Appl. Probab. 11 (1979), no. 4, $750-783$.

Wang, D. Finite-time ruin probability with heavy-tailed claims and constant interest rate. Stoch. Models 24 (2008), no. 1, 41-57.

Wang, D.; Tang, Q. Tail probabilities of randomly weighted sums of random variables with dominated variation. Stoch. Models 22 (2006), no. 2, 253-272. 